\title{
Espacios de Whyburn
}

\author{
Maira Madriz Mendoza \\ Asesor: Dr. Richard Wilson R.
}

Octubre 2007 

A mis padres 



\section{Índice general}

Con Profunda Intención VII

Introducción

1. Nociones Fundamentales 1

1.1. Preliminares . . . . . . . . . . . . . . . . . . 1

Algunos Cardinales Importantes . . . . . . . . . . . . . 7

1.2. Monoliticidad . . . . . . . . . . . . . . . . . 9 9

2. Espacios de Whyburn y Débilmente Whyburn 11

2.1. Espacios de Whyburn bajo ciertos tipos de Funciones Continuas 14

2.2. Espacios Pseudocompactos de Whyburn . . . . . . . . . . 23

2.3. P-espacios . . . . . . . . . . . . . . 30

2.4. Una Caracterización en Espacios Linealmente Ordenados . . . 34

2.5. Propiedad de Whyburn en Productos . . . . . . . . . . 38

2.6. Propiedad de Whyburn y Estrechez de Abanico . . . . . . . . 43

3. Espacios $C_{p}(X)$

Una caracterización para que $C_{p}(\xi)$ sea débilmente Whyburn . 51

$\begin{array}{ll}\text { Preguntas Abiertas } & 59\end{array}$

$\begin{array}{ll}\text { Bibliografía } & 63\end{array}$ 



\section{Con Profunda Intención}

Posiblemente estas líneas no expresen la aventura que ha significado esta obra y tampoco lo agradecida que me siento. Cada página de este trabajo contiene un cúmulo de emociones que en conjunto contienen mi propia esencia y la esperanza ante el futuro, como si hubiera logrado algo sorprendente aunque estoy conciente de que este camino no tiene fin.

Como siempre, me gustaría empezar agradeciéndole a Dios por ponerme en este camino y por permitirme llegar a este momento de la mejor manera.

A mis padres, por su infinito amor y apoyo incondicional. Sobre todo porque son y serán los mejores cimientos, quienes confian y creen en mí.

A mis hermanos: Allan, Gaby y Héctor, por estar a mí lado, por su peculiar amor y muchos momentos divertidos. A Brenda por su particular forma de ser y al pequeño Emilio, que con su presencia me hace tan feliz e irradia cada momento de mi vida.

A Gerardo por estar a mi lado, por consentirme y animarme todo el tiempo, aún cuando no tiene idea de lo que hago.

A mis amigos: Álgebra, Alejandra, Nancy, Yesenia y David, por ser mis amigos más leales y a quienes llevo en el corazón. También quiero agradecer a mis amigos de la UAM, quienes han visto mi desarrollo y me han tolerado en múltiples ocasiones: Alex, Juan Carlos, Janet, Marco, Perla, Rosa, Sol y Tere; así como a algunos investigadores que han sido piezas clave en mi formación y a quienes prefiero no mencionar explícitamente para evitar omitir a alguien.

A Richard le agradezco su disposión, entrega y amistad, pero sobre todo, le agradezco por su extraordinaria paciencia y el que nunca me ha dejado sola.

A mis sinodales, Dr. Vladimir V. Tkachuk y Dr. Adalberto García-Maynez, 
por tomarse el tiempo de leer y mejorar mi trabajo, con comentarios precisos y alentadores.

A la Universidad Autónoma Metropolitana por adoptarme y abrirme las puertas de un nuevo sendero.

Al Consejo Nacional de Ciencia y Tecnología, por ser mi sustento mientras realicé la maestría, ya que sin su generoso apoyo, el camino hubiese sido escabroso.

Para concluir no quiero dejar en mi mente lo esencial, gracias a usted amable lector, por considerar este trabajo. 


\section{Introducción}

Si $X$ es un espacio topológico y $F \subset X$, nos permitimos decir que $F$ es casi cerrado si $c l_{X}(F) \backslash F$ es un conjunto de un sólo punto. El hecho de que $c l_{X}(F) \backslash F=\{x\}$, se denota por $F \rightarrow x$. Es natural decir que la topología de un espacio $X$ está determinada por subespacios casi cerrados si, para cualquier conjunto no cerrado $A \subset X$ y cualquier $x \in c l_{X}(A) \backslash A$, existe un conjunto casi cerrado $F \subset A$ tal que $F \rightarrow x$. Por analogía con los conceptos de Fréchet-Urysohn y espacios secuenciales, decimos que un espacio topológico $X$ es débilmente determinado por subespacios casi cerrados si, para cualquier conjunto no cerrado $A \subset X$, existe un conjunto casi cerrado $B \subset A$ tal que $B \rightarrow x \notin A$.

Los espacios determinados por subespacios casi cerrados fueron introducidos primero por Whyburn en [22], quien los bautizó como espacios de accesibilidad y estudió las propiedades de mapeos pseudoabiertos en espacios de accesibilidad. Con el paso del tiempo estos espacios fueron cambiando de nombre, incluso cobran vida en la topología de categorías en [19] y no fue sino hasta hace poco tiempo que los especialistas decidieron dar crédito a Whyburn y llamar a los espacios determinados por subespacios casi cerrados, espacios de Whyburn. Obviamente, los espacios débilmente determinados por subespacios casi cerrados adquirieron el nombre de espacios débilmente de Whyburn.

El propósito de este trabajo tiene la finalidad de unificar los diversos aspectos que se han estudiado de las propiedades de Whyburn y débilmente Whyburn. Los artículos de investigación se escriben para especialistas, en éstos se suponen dominados todos los cimientos necesarios para entenderlos. Es por ello que intentamos hacer comprensibles los resultados expuestos para un mayor número de lectores.

El trabajo aquí presentado, está distribuido básicamente en tres capítulos. En el primero, se exponen algunas funciones cardinales y resultados generales 
que son necesarios para abordar los teoremas principales en los capítulos posteriores.

El segundo capítulo constituye la columna vertebral del presente trabajo, pues está dedicado fundamentalmente a probar los resultados hasta ahora conocidos. Se estudian propiedades básicas de los espacios en cuestión, que son conocidas pero que no suelen probarse. En particular, se estudian las condiciones para que un espacio Whyburn o débilmente Whyburn sea Fréchet; además de su estrecha relación con los conceptos de radialidad, pseudoradialidad y conceptos relacionados. Se dan condiciones suficientes para que el producto de dos espacios compactos sea débilmente Whyburn. Por otro lado, se construye en ZFC un ejemplo de un espacio de Tychonoff, cero-dimensional, pseudocompacto y disperso que es Whyburn pero que tiene estrechez no numerable. También se estudia la propiedad de Whyburn en $P$-espacios y se da una caracterización de dicha propiedad en espacios linealmente ordenados. Por otro lado, se contesta negativamente a la pregunta hecha en [18] de si ¿es cierto que cualquier $P$-espacio es débilmente Whyburn?

En el tercer capítulo, se prueba que si $X$ es paracompacto y $C_{p}(X)$ es un espacio Whyburn, entonces $X$ es de Hurewicz. En especial, nos dedicamos a caracterizar a los ordinales $\xi$ para los cuales $C_{p}(\xi)$ es débilmente Whyburn.

Este trabajo también incluye algunas de las preguntas abiertas que hay en este campo. 


\section{Capítulo 1}

\section{Nociones Fundamentales}

En el presente capítulo estableceremos las nociones y resultados fundamentales que vamos a utilizar. Iniciaremos definiendo algunas funciones cardinales y, en lo sucesivo, presentaremos conceptos y resultados básicos de la propiedad que motiva este trabajo.

A menudo usaremos resultados y terminología, que sin lugar a dudas, el lector familiarizado recordará. Cuando usemos algún resultado en el que no incluyamos la prueba, daremos la referencia necesaria.

\subsection{Preliminares}

En esta sección presentamos algunas funciones cardinales y resultados que ocuparemos posteriormente. Para empezar definiremos algunas funciones cardinales.

1.1 Definición. El conjunto de números cardinales de la forma $|\mathcal{B}|$, donde $\mathcal{B}$ es una base para un espacio topológico $X$, tiene un elemento más pequeño; este número cardinal es llamado el peso del espacio $X$ y es denotado por $w(X)$.

1.2 Definición. El carácter de un punto $x$ en un espacio topológico $X$ se define como el número cardinal mínimo de la forma $|B(x)|$, donde $B(x)$ es una base local de vecindades de $x$; este número cardinal es denotado por $\chi(x, X)$. El carácter de $X$ se define como $\chi(X)=\sup \{\chi(x, X): x \in X\}$. 
1.3 Definición. El pseudocarácter de un punto $x$ en un espacio $X \in T_{1}$ se define como el número cardinal más pequeño de la forma $|\mathcal{U}|$, donde $\mathcal{U}$ es una familia de subconjuntos abiertos de $X$ tales que $\bigcap \mathcal{U}=\{x\}$; este número cardinal es denotado por $\psi(x, X)$. El pseudocarácter de $X$ se define como $\psi(X)=\sup \{\psi(x, X): x \in X\}$.

1.4 Definición. Una familia $\mathcal{N}$ de subconjuntos de un espacio topológico $X$ es una red para $X$ si para cualquier punto $x \in X$ y cualquier vecindad $U$ del punto $x$ existe $M \in \mathcal{N}$ tal que $x \in M \subset U$. Así que, cualquier base para $X$ es una red para $X$. El peso red de un espacio $X$ se define como $n w(X)=\operatorname{mín}\{|\mathcal{N}|: \mathcal{N}$ es una red para $X\}$.

1.5 Definición. El mínimo cardinal $\kappa$ tal que cualquier cubierta abierta de un espacio $X$ tiene una subcubierta abierta de cardinalidad $\leq \kappa$, es llamado número de Lindelöf del espacio $X$ y es denotado por $L(X)$. El número hereditariamente Lindelöf se define como $h L(X)=\sup \{L(Y): Y \subseteq X\}$.

1.6 Definición. La estrechez de $X$ en un punto $x \in X$ es el menor número cardinal $m \geq \aleph_{0}$ con la propiedad de que, si $C \subseteq X$ y $x \in c_{X}(C)$ entonces existe un conjunto $C_{0} \subset C$ tal que $\left|C_{0}\right| \leq m$ y $x \in c l_{X}\left(C_{0}\right)$, este número cardinal es denotado por $t(x, X)$. La estrechez de un espacio $X$ se denota por $t(X)=\sup \{t(x, X): x \in X\}$.

1.7 Lema. Para cualquier subespacio $Y$ de un espacio $X$, se tiene que $n w(Y) \leq n w(X)$.

Demostración. Si $\mathcal{N}$ es una red de $X$, entonces $\{Y \cap N: N \in \mathcal{N}\}$ es una red en $Y$.

1.8 Proposición. $n w(X) \geq L(X)$.

Demostración. Sean $n w(X)=\kappa$, una familia $\mathcal{N}=\left\{B_{\alpha}: \alpha<\kappa\right\}$ una red en $X$ y $\mathcal{U}$ una cubierta abierta de $X$. Entonces, cada $U \in \mathcal{U}$ se puede expresar como $U=\bigcup_{\alpha \in J_{U} \subset \kappa} B_{\alpha}$. Por lo que $\mathcal{G}=\left\{B_{\alpha}: \alpha \in J_{U}\right.$ y $\left.U \in \mathcal{U}\right\}$ es una cubierta de $X$ y $|\mathcal{G}| \leq \kappa$. Para cada $\alpha \in J_{U}$ y $U \in \mathcal{U}$, escogemos $U_{\alpha} \in \mathcal{U}$ tal que $B_{\alpha} \subseteq U_{\alpha}$. Luego, $\left\{U_{\alpha}: \alpha<\kappa\right\}$ es una subcubierta abierta de cardinalidad $\kappa$. En conclusión, se ha demostrado que $n w(X)=\kappa$ implica que $L(X) \leq \kappa$. Por el Lema 1.7, $h L(X) \leq \kappa$ 
1.9 Teorema. Si $X$ es un espacio de Hausdorff entonces $\psi(X) \leq h L(X)$.

Demostración. Sean $x \in X$ y $h L(X)=\kappa$. Como $X$ es de Hausdorff, para cualquier $y \in X$ con $y \neq x$, existe una vecindad $U_{y}$ de $y$ tal que $x \notin c l_{X}\left(U_{y}\right)$. Luego, $X \backslash\{x\}$ se puede cubrir con $\left\{U_{y_{\alpha}}: \alpha<\kappa\right\}$. En consecuencia, $\{x\}=$ $\bigcap_{\alpha<\kappa}\left(X \backslash c l_{X}\left(U_{y_{\alpha}}\right)\right)$. Por tanto, $\psi(X) \leq \kappa$.

1.10 Corolario. En un espacio de Hausdorff $X, \psi(X) \leq h L(X) \leq n w(X)$.

Demostración. Es consecuencia de la Proposición 1.8 y del Teorema 1.9.

1.11 Teorema ([14]). En un espacio compacto y de Hausdorff $X$, se cumple que $\psi(X)=\chi(X)$. En particular, cualquier espacio compacto con pseudocarácter numerable es primero numerable. (Ver [14], Teorema 7.1)

Aún cuando los siguientes conceptos son muy conocidos, se los recordamos al lector debido a que dan pie a varios de los resultados más importantes.

Cabe señalar que a cualquier función $S: \alpha \longrightarrow X$ donde $\alpha$ es cualquier ordinal le llamaremos cadena y, en adelante, se denotará en la forma $\left\{x_{\beta}\right.$ : $\beta<\alpha$, donde $S(\beta)=x_{\beta}$. Reservamos el término sucesión para cadenas de longitud numerable.

La cadena $\left\{x_{\beta}: \beta<\alpha\right\}$ se dice que converge a un punto $x$, si para cualquier vecindad $U$ de $x$, existe algún $\gamma<\alpha$ tal que $x_{\beta} \in U$ para cada $\beta \geq \gamma$.

1.12 Definición. Un espacio $X$ es Fréchet-Urysohn, si para cualquier subconjunto $A \subset X$ y para cualquier $x \in c_{X}(A)$ existe una sucesión $S \subset A$ que converge a $x$.

1.13 Definición. Un espacio $X$ es secuencial, si para cualquier subconjunto no cerrado $A \subset X$ existe una sucesión $S \subset A$ que converge a algún $x \in \operatorname{cl}_{X}(A) \backslash A$.

1.14 Definición. Un subconjunto $A$ de un espacio $X$ es $\kappa$-cerrado (respectivamente, $<\kappa$-cerrado) si $B \subset A y|B| \leqslant \kappa$ (respectivamente, $|B|<\kappa$ ) implican que $\operatorname{cl}_{X}(B) \subset A$. 
1.15 Proposición. Si $A \subseteq X$ es $<\kappa$-cerrado y $f: X \longrightarrow Y$ es un mapeo cerrado, entonces $f(A)$ es $<\kappa$-cerrado en $Y$.

Demostración. Sea $B \subseteq f(A)$ tal que $|B|<\kappa$. Para cada $y \in B$ escogemos $x_{y} \in A$ tal que $f\left(x_{y}\right)=y$. Sea $C=\left\{x_{y}: y \in B\right\}$. Entonces $|C|<\kappa$ y $f(C)=B$. Por hipótesis, $c l_{X}(C) \subset A$. Luego, $f\left(c l_{X}(C)\right) \subseteq$ $c l_{Y}(f(C)) \subseteq c_{Y}\left(f\left(c l_{X}(C)\right)\right)=f\left(c l_{X}(C)\right)$, donde la primera contención es por continuidad y la igualdad se debe a que $f$ es cerrada. De esta manera, $c l_{Y}(B)=c l_{Y}(f(C))=f\left(c l_{X}(C)\right) \subset f(A)$, lo que culmina la prueba.

Los siguientes conceptos son interesantes por si mismos, no obstante, nos enfocaremos en las importantes conexiones que hay con las propiedades que estudiaremos.

1.16 Definición. Un espacio $X$ es semiradial siempre que para cualquier conjunto no $\kappa$-cerrado $A \subset X$, existe una cadena $\left\{x_{\xi}: \xi \in \lambda\right\} \subset A$, con $\lambda \leqslant \kappa$, que converge a un punto en $X \backslash A$.

1.17 Definición. Un espacio $X$ es pseudoradial, si cualquier conjunto no cerrado $A \subset X$ contiene una cadena que converge a un punto en $X \backslash A$.

1.18 Definición. Un espacio $X$ es radial, si cualquier punto en la cerradura de un subconjunto $A \subset X$ es límite de una cadena en $A$.

Nótese que radial implica semiradial y semiradial implica pseudoradial. Además, cualquier espacio hereditariamente pseudoradial es radial. Por otro lado, los espacios secuenciales son pseudoradiales y los espacios de Fréchet son radiales. Un espacio radial no necesariamente es secuencial.

Ahora nos ocuparemos de resultados diversos, que no tienen relación propiamente, pero que nos servirán como herramienta en los siguientes capítulos.

1.19 Definición. Si $X$ es un espacio topológico y $F \subset X$, decimos que $F$ es casi cerrado si cl ${ }_{X}(F) \backslash F$ es un conjunto de un sólo punto.

1.20 Definición. La $\omega$-modificación de un espacio $(X, \tau)$ es el espacio cuya topología está generada por los subconjuntos $G_{\delta}$ de $(X, \tau)$. 
1.21 Lema. Si $X$ es un espacio de Hausdorff e infinito, entonces contiene a un subconjunto discreto infinito.

Demostración. Sean $x, y \in X$ distintos. Entonces existe una vecindad abierta $U$ de $x$ tal que $y \notin c l_{X}(U)$. Así que, $U$ o bien $X \backslash c l_{X}(U)$ es infinito. Sin pérdida de generalidad, supongamos que $U$ es infinito. De modo que existe un punto $x_{1} \in U$ distinto de $x$. Luego, existe una vecindad abierta $V_{1}$ de $x$ tal que $x_{1} \notin c l_{X}\left(V_{1}\right)$. De aquí, $V_{1}$ o $U \backslash c l_{X}\left(V_{1}\right)$ es infinito, digamos $V_{1}$. Similarmente, existe $x_{2} \in V_{1}$ y un abierto infinito $V_{2}$ de $x$ en $V_{1}$ tal que $x_{2} \notin c l_{X}\left(V_{2}\right)$. $\mathrm{Al}$ proceder inductivamente, se obtiene que el conjunto $\left\{x_{n}: n \in \omega\right\}$ es el subconjunto discreto requerido.

1.22 Definición. Un espacio de Hausdorff $X$ se llama $H$-cerrado, si $X$ es cerrado en cualquier espacio de Hausdorff que lo contenga.

Un ejemplo que se empleará en el presente trabajo es precisamente un espacio $H$-cerrado. Así que sólo mencionamos algunas equivalencias del mismo para ilustrar el concepto, para una mejor referencia, véase ([11], 3.12.5).

1.23 Teorema ([11]). Sea $X$ un espacio de Hausdorff. Las siguientes condiciones son equivalentes:

(a) $X$ es H-cerrado.

(b) Para cualquier familia $\left\{V_{s}\right\}_{s \in S}$ de subconjuntos abiertos de $X$ que tienen la propiedad de la intersección finita, la intersección $\bigcap_{s \in S} c l_{X}\left(V_{s}\right)$ es no vacía.

(c) Cualquier ultrafiltro en la familia de todos los subconjuntos abiertos de $X$ converge.

(d) Cualquier cubierta abierta $\left\{U_{s}\right\}_{s \in S}$ del espacio $X$ contiene una subfamilia finita $\left\{U_{s_{1}}, \ldots, U_{s_{k}}\right\}$ tal que $c l_{X}\left(U_{s_{1}}\right) \cup \cdots \cup c l_{X}\left(U_{s_{k}}\right)=X$

Una colección de subconjuntos abiertos, no vacíos y disjuntos dos a dos en $X$ es llamada familia celular. 
1.24 Lema. Sean $(X, \tau)$ un espacio de Hausdorff infinito y $x \in X$. Existe una familia celular $\gamma$ infinita tal que para cada $U \in \gamma, x \notin c l_{X}(U)$. Además, $\gamma$ se puede extender a una familia maximal celular con dicha propiedad.

Demostración. Sean $x_{1}, x_{2} \in X$ con $x_{1} \neq x_{2}$ y distintos de $x$. Entonces existen conjuntos abiertos ajenos $V, V_{1}, V_{2} \subset X$ tales que $x \in V, x_{1} \in V_{1}$ y $x_{2} \in V_{2}$, de manera que $x \notin c l_{X}\left(V_{1}\right) \cup c l_{X}\left(V_{2}\right)$. Si $V_{1}$ o $V_{2}$ es finito, digamos $V_{2}$, ponemos $U_{1}=V_{2}$ y repetimos el argumento en el abierto infinito $V_{1}$. Si $V_{1}$ y $V_{2}$ son finitos, entonces son abiertos y cerrados, así que ponemos $U_{1}=V_{1}$ y repetimos el argumento en $X \backslash V_{1}$. Procediendo de esta manera se obtiene la familia $\gamma=\left\{U_{i}: i \in I\right\}$ requerida.

Sea $\mathcal{P}=\left\{\gamma: \gamma\right.$ es una familia celular y $x \notin c l_{X}(U)$ para cada $\left.U \in \gamma\right\}$. Dadas $\gamma, \gamma^{\prime} \in \mathcal{P}$ hacemos $\gamma \leq \gamma^{\prime}$ si $\gamma \subset \gamma^{\prime}$. Esto hace que el conjunto $\mathcal{P}$ sea parcialmente ordenado. A continuación probaremos que $(\mathcal{P}, \leq)$ tiene todas sus cadenas acotadas.

Sea $\mathcal{C}$ una cadena en $\mathcal{P}$. Si $\mu=\bigcup \mathcal{C}$, entonces $\gamma \leq \mu$ para todo $\gamma \in \mathcal{C}$, así que basta establecer que $\mu \in \mathcal{P}$ para cerciorarnos de que la cadena $\mathcal{C}$ es acotada en $\mathcal{P}$. Si $U \in \mu$ entonces $U \in \gamma$ para alguna $\gamma \in \mathcal{C}$. Como $\gamma$ es una familia celular con la propiedad requerida, se tiene que $x \notin c l_{X}(U)$. Así que basta verificar que $\mu$ es una familia celular. Sean $U, V \in \mu$ con $U \neq V$. Entonces existen $\gamma_{U}, \gamma_{V} \in \mathcal{C}$ tales que $U \in \gamma_{U}$ y $V \in \gamma_{V}$. Como $\mathcal{C}$ es una cadena, tenemos que $\gamma_{U} \subset \gamma_{V}$ o $\gamma_{V} \subset \gamma_{U}$. Si $\gamma_{U} \subset \gamma_{V}$ entonces $U, V \in \gamma_{V}$. De manera que $U, V$ son abiertos, ajenos y no vacíos. Análogamente, si $\gamma_{V} \subset \gamma_{U}$. Luego, $\mathcal{P}$ tiene todas sus cadenas acotadas. Por el Lema de Zorn, concluimos que $\mathcal{P}$ tiene un elemento $\xi$ que es maximal en $\mathcal{P}$.

1.25 Definición. Un espacio $X$ es discretamente generado, si para cualquier $A \subset X$ y $x \in \operatorname{cl}_{X}(A)$ existe un subespacio discreto $D \subset A$ tal que $x \in c l_{X}(D)$.

1.26 Proposición. Si L es un espacio linealmente ordenado, entonces es discretamente generado.

Demostración. Sean $A \subset L$ con $|A|=\kappa$ y $x \in \operatorname{cl}_{L}(A) \backslash A$. Entonces $x \in$ $c l_{L}\left(A \cap(\leftarrow, x)_{L}\right)$ o $x \in c l_{L}\left(A \cap(x, \rightarrow)_{L}\right)$. Como los casos son idénticos, sin perder generalidad podemos suponer que $x \in \mathrm{cl}_{L}\left(A \cap(\leftarrow, x)_{L}\right)$. Escojamos $x_{0} \in A$ y supongamos que ya se ha elegido $x_{\beta}$ para cada $\beta<\alpha$ tal que $\left\{x_{\beta}: \beta<\alpha\right\} \subseteq A \cap(\leftarrow, x)_{L}$ es discreto. Tenemos dos posibilidades: 
a) Si $x \in c l_{L}\left(\left\{x_{\beta}: \beta<\alpha\right\}\right)$, ponemos $D_{\alpha}=\left\{x_{\beta}: \beta<\alpha\right\}$.

b) Si $x \notin c l_{L}\left(\left\{x_{\beta}: \beta<\alpha\right\}\right)$, entonces existe $a<x$ tal que $(a, \rightarrow) \cap$ $\left\{x_{\beta}: \beta<\alpha\right\}=\emptyset$. Ahora bien, $(a, x) \neq \emptyset$, pues de lo contrario $x \notin$ $c l_{L}\left(A \cap(\leftarrow, x)_{L}\right)$. Por lo tanto, existe $x_{\alpha} \in(a, x)$ y, en consecuencia, $\left\{x_{\beta}\right.$ : $\beta \leq \alpha\}$ es discreto. Para algún ordinal $\kappa$ el caso a) ocurre, ya que de lo contrario $(\sup (A), x)$ sería una vecindad de $x$ ajena a $A$.

Finalmente, un Teorema importante en la Teoría de Conjuntos es el llamado Teorema del $\Delta$-sistema, (ver [14], Teorema 2.4). A saber,

1.27 Teorema ([15]). Sea $\mathcal{A}$ una familia de conjuntos con $|\mathcal{A}|=\kappa y|A| \leq \lambda$ para cada $A \in \mathcal{A}$, donde $\kappa>\omega$ es regular y $\mu^{\lambda}<\kappa$ para cualquier $\mu<\kappa$. Entonces existe un conjunto $D$ y una subfamilia $\mathcal{A}^{\prime} \subset \mathcal{A}$ con $\left|\mathcal{A}^{\prime}\right|=|\mathcal{A}|$ tal que la intersección de cualesquiera dos elementos distintos de $\mathcal{A}^{\prime}$ es $D$.

\section{Algunos Cardinales Importantes}

En esta subsección se definen algunos cardinales importantes asociados con $\omega$ que en general son vistos desde la perspectiva de la Teoría de Conjuntos.

1.28 Definición. Cualesquiera dos conjuntos infinitos son casi disjuntos si su intersección es finita. Una familia casi ajena es un conjunto en que cualesquiera dos de sus elementos distintos son casi disjuntos. Una familia maximal casi ajena es una familia casi ajena $\mathcal{A}$ que no está contenida propiamente en otra familia $\mathcal{B}$ casi ajena.

Dados dos conjuntos infinitos $A$ y $B$ decimos que $A \subset^{*} B$ si $A \backslash B$ es finito. Si $f, g \in \omega^{\omega}$ entonces $f<^{*} g$, si existe $\kappa \in \omega$ tal que $f(n)<g(n)$ para toda $n \geq \kappa$.

1.29 Definición. Un conjunto $F \subset \omega^{\omega}$ es acotado si existe un $g \in \omega^{\omega}$ tal que $f<^{*} g$ para cualquier $f \in F$.

El símbolo $\mathfrak{b}$ denotará la mínima cardinalidad de una familia no acotada. 
1.30 Lema. Todo subconjunto numerable de $\left(\omega^{\omega},<^{*}\right)$ es acotado.

Demostración. Supongamos que $\left\{f_{m}: m \in \omega\right\}$ es una familia numerable. Definimos $f: \omega \rightarrow \omega$ por $f(n)=\sup \left\{f_{0}(n), \ldots, f_{n}(n)\right\}+1$. Entonces, $f_{n}(m)<f(m)$ para cada $m \geq n$. Por tanto, $f_{n}<^{*} f$ para cada $n \in \omega$.

1.31 Definición. Una familia $D \subset \omega^{\omega}$ es dominante, si para cualquier $f \in \omega^{\omega}$ existe $g \in D$ tal que $f \leq^{*} g$.

El símbolo $\mathfrak{d}$ denotará la mínima cardinalidad de una familia dominante.

1.32 Observación. Cualquier familia dominante es no acotada.

1.33 Corolario. En $Z F C, \omega<\mathfrak{b} \leq \mathfrak{d} \leq \mathfrak{c}=2^{\omega}=\omega^{\omega}$

Demostración. Es consecuencia inmediata del Lema 1.30 y de la Observación 1.32 .

Cabe señalar que la igualdad $\mathfrak{b}=\mathfrak{d}$ es una consecuencia inmediata de la Hipótesis del Continuo, pero también se sigue del Axioma de Martin (ver [10, Corolario 3.5]), de manera que la afirmación $\mathfrak{b}=\mathfrak{d}$ es compatible con los axiomas usuales de ZFC.

1.34 Lema. Si $\mathfrak{b}=\mathfrak{d}$, entonces existe una familia dominante bien ordenada en $\left(\omega^{\omega},<^{*}\right)$.

Demostración. Sea $\Phi=\left\{\phi_{\alpha}: \alpha<\mathfrak{b}\right\}$ una familia dominante. Definimos a la familia $\left\{\psi_{\alpha}: \beta \in \mathfrak{b}\right\}$ como sigue: Sea $\psi_{0}=\phi_{0}$; una vez elegida $\psi_{\beta}$ para toda $\beta<\alpha$, escogemos $\psi_{\alpha}$ como sigue:

Si $\alpha$ no es límite, entonces $\alpha=\delta+1$ para alguna $\delta$ y ponemos $\psi_{\alpha}=\phi_{\alpha} \vee \psi_{\delta}$. De manera que, $\psi_{\alpha} \geq \psi_{\delta}$.

Si $\alpha$ es límite, entonces $\left\{\psi_{\beta}: \beta<\alpha\right\}$ es acotada debido a que la familia tiene cardinal menor que $\mathfrak{b}$. Entonces existe $f_{\alpha} \in \omega^{\omega}$ tal que $f_{\alpha}>^{*} \psi_{\beta}$ para cada $\beta<\alpha$. De modo que ponemos $\psi_{\alpha}=f_{\alpha} \vee \phi_{\alpha}$, lo que implica que $\psi_{\alpha} \geq^{*} \psi_{\beta}$ para toda $\beta<\alpha$ y $\psi_{\alpha} \geq \phi_{\alpha}$.

La familia $\left\{\psi_{\alpha}: \alpha<\mathfrak{b}\right\}$ es dominante, debido a que $\psi_{\alpha} \geq \phi_{\alpha}$ para toda $\alpha$ y $\psi_{\beta} \geq^{*} \psi_{\alpha}$ para toda $\alpha<\beta<\mathfrak{b}$. 


\subsection{Monoliticidad}

1.35 Definición. Un espacio $X$ tiene estrechez de abanico numerable siempre que para cualquier punto $x \in X$ y cualquier familia numerable $\left\{G_{n}\right.$ : $n \in \omega\}$ de subconjuntos de $X$, si $x \in c_{X}\left(G_{n}\right)$ para cualquier $n \in \omega$ existen subconjuntos finitos $F_{n} \subset G_{n}$ tales que $x \in c l_{X}\left(\bigcup_{n \in \omega} F_{n}\right)$.

1.36 Definición. Un espacio $X$ es $\omega$-monolítico si para cada conjunto numerable $A \subseteq X, n w\left(c l_{X}(A)\right) \leq \omega$.

1.37 Definición. Un espacio $X$ es $\omega-\psi$-monolítico si la cerradura de cualquier subconjunto numerable tiene pseudocarácter numerable (en si mismo).

1.38 Proposición. Un espacio $\omega$-monolítico es $\omega-\psi$-monolítico.

Demostración. Sea $A$ cualquier subespacio numerable de un espacio $\omega$-monolítico $X$. Entonces, $n w\left(c l_{X}(A)\right) \leq \omega$. Por tanto, por la Proposición 1.10, $X$ es $\omega-\psi$-monolítico. 



\section{Capítulo 2}

\section{Espacios de Whyburn y Débilmente Whyburn}

En este capítulo, introduciremos los conceptos que dan origen al presente trabajo.

2.1 Definición. Un espacio $X$ es de Whyburn siempre que para cualquier conjunto no cerrado $A \subset X$ y para todo $x \in c_{X}(A) \backslash A$ existe un subconjunto $B \subset A$ tal que $\operatorname{cl}_{X}(B) \backslash A=\{x\}$.

2.2 Definición. Un espacio $X$ es débilmente Whyburn siempre que para cualquier conjunto no cerrado $A \subset X$ existe un subconjunto $B \subset A$ tal que $\left|c l_{X}(B) \backslash A\right|=1$.

Por tanto, todo espacio de Whyburn es débilmente Whyburn.

2.3 Definición. Un subconjunto A de un espacio $X$ es Whyburn cerrado, si para cualquier $F \subset A$ se tiene que $\left|c l_{X}(F) \backslash A\right| \neq 1$.

Obsérvese que un espacio $X$ es débilmente Whyburn si y sólo si cualquier subconjunto Whyburn cerrado de $X$ es cerrado.

2.4 Proposición. En un espacio de Hausdorff X, se cumple lo siguiente:

1. Si $X$ es un espacio Fréchet-Urysohn, entonces es Whyburn.

2. Si $X$ es un espacio secuencial, entonces es débilmente Whyburn. 
Demostración. Basta observar que en cualquier espacio de Hausdorff, cualquier sucesión tiene un único punto límite.

A continuación se exhiben algunos resultados elementales referentes a las nociones anteriores.

2.5 Proposición. Cualquier subespacio de un espacio Whyburn es Whyburn.

Demostración. Sean $X$ un espacio Whyburn y $Y \subset X$. Si $A$ no es cerrado en $Y$, entonces no es cerrado en $X$. Escogemos $y \in c l_{Y}(A) \backslash A$. Puesto que $y \in c l_{X}(A) \backslash A$, existe $D \subset A$ tal que $c l_{X}(D) \backslash A=\{y\}$. Luego, $c l_{Y}(D) \backslash A=$ $\{y\}$. Por tanto, $Y$ es Whyburn.

Cabe señalar que en general la propiedad de ser débilmente Whyburn al igual que sucede para espacios secuenciales, no es hereditaria. Más adelante, en 2.61, veremos un ejemplo de un subespacio de un espacio débilmente Whyburn que no es débilmente Whyburn.

2.6 Proposición ([21]). Cualquier subespacio abierto o cerrado de un espacio débilmente Whyburn es débilmente Whyburn.

Demostración. Supongamos que $X$ es un espacio débilmente Whyburn y $Y \subset X$ es abierto. Sea $A$ un subconjunto no cerrado de $Y$. Entonces el conjunto $A \cup(X \backslash Y)$ no es cerrado en $X$. Por hipótesis, existe un subconjunto $F \subset[A \cup(X \backslash Y)]$ tal que $c l_{X}(F) \backslash[A \cup(X \backslash Y)]=\{q\}$. Luego, $G=F \cap A \subset A$ y $c l_{Y}(G) \backslash A=c l_{X}(F \cap A) \cap(Y \cap(X \backslash A)) \subset c l_{X}(F) \backslash[A \cup(X \backslash Y)]=\{q\} ;$ además, como $\{q\} \subset c l_{Y}(G) \backslash A$, se tiene que $Y$ es débilmente Whyburn.

Sea $Z \subset X$ cerrado; supongamos que $A \subset Z$ no es cerrado en $Z$. Entonces $A$ no es cerrado en $X$. Puesto que $X$ es débilmente Whyburn, existe $B \subset A$ tal que $c l_{X}(B) \backslash A=\{p\}$. Esto implica que $c l_{Z}(B) \backslash A=\{p\}$. Por tanto, $Z$ es débilmente Whyburn.

Un espacio ordenado generalizado (GO) es un conjunto linealmente ordenado $(X,<)$ cuya topología está generada por intervalos (de cualquier tipo). Si la topología de $X$ coincide con la topología de intervalos abiertos con el orden dado, entonces decimos que $X$ es un espacio topológico linealmente ordenado (LOTS). Se sabe que un espacio GO es un subespacio de un espacio LOTS con la topología relativa. 
2.7 Proposición ([20]). Si X es un espacio ordenado generalizado entonces es débilmente Whyburn.

Demostración. Sean $A \subseteq X$ y $x \in c l_{X}(A) \backslash A$. Entonces $x \in c l_{X}(A \cap(x, \rightarrow))$ o bien $x \in c l_{X}(A \cap(\leftarrow, x))$. Como los casos son idénticos, supongamos sin perder generalidad que $x \in \operatorname{cl}_{X}(A \cap(\leftarrow, x))$, entonces podemos escoger una cadena ascendente $\left\{x_{\alpha}: \alpha<\kappa\right\} \subseteq A \cap(\leftarrow, x)$ tal que $\sup _{\alpha<\kappa} x_{\alpha}=x$. Sea $\xi(x)$ la mínima cardinalidad de una cadena en $A$ que converge a $x$ y denotamos por $\kappa=\min \left\{\xi(z): z \in c l_{X}(A) \backslash A\right\}$. Para $y \in c l_{X}(A) \backslash A \operatorname{con} \xi(y)=\kappa \mathrm{y}$ $B=\left\{x_{\alpha}: \alpha<\kappa\right\} \subset A$ se tiene que $c l_{X}(B) \backslash A=\{y\}$, pues de lo contrario existe $s \in\left(c l_{X}(A) \backslash A\right) \cap c l_{X}(B)$ con $s \neq y$. Como $B$ converge a $y$, existe algún $\gamma<\kappa$ tal que $s \in \operatorname{cl}_{X}\left(\left\{x_{\alpha}: \alpha<\gamma\right\}\right)$, lo que contradice la minimalidad de $\kappa$.

Puesto que un subespacio de un espacio ordenado generalizado es un espacio ordenado generalizado, se sigue que un espacio GO es hereditariamente débilmente Whyburn.

Sabemos que los espacios Fréchet-Urysohn son precisamente los espacios hereditariamente secuenciales ([3]). No obstante, los espacios hereditariamente débilmente Whyburn no son necesariamente de Whyburn como veremos a continuación; sin embargo, como consecuencia inmediata de la definición, se tiene la siguiente:

2.8 Proposición ([21]). Cualquier espacio Whyburn es un espacio hereditariamente débilmente Whyburn.

Para ilustrar que el recíproco de la proposición anterior no siempre se cumple, veamos un ejemplo.

2.9 Ejemplo. El ordinal $\omega_{1}+1$ con la topología del orden es hereditariamente débilmente Whyburn pero no es Whyburn.

Demostración. Sea $A=\left\{\alpha: \alpha<\omega_{1}\right.$ es ordinal sucesor $\}$ un conjunto no cerrado de $\omega_{1}+1$. Supongamos que existe $B \subset A$ tal que $\omega_{1} \in c l_{\omega_{1}+1}(B)$. Entonces $|B|=\omega_{1}$, pues $B$ es cofinal. Ahora, escogemos $b \in B$ tal que $C=\{\alpha: \alpha<b$ y $\alpha \in B\}$ sea un subconjunto infinito de $\omega_{1}$. Puesto que $\omega_{1}$ es numerablemente compacto, $C$ tiene un punto de acumulación en $\left(\omega_{1}+1\right) \backslash A$, de donde, $\left|c l_{\omega_{1}+1}(B) \backslash A\right| \neq 1$. Por tanto, $\omega_{1}+1$ no es Whyburn.

No obstante, por la Proposición 2.7, $\omega_{1}+1$ es hereditariamente débilmente Whyburn. 
2.10 Lema ([20]). Cualquier espacio con un único punto no aislado es de Whyburn.

Demostración. Sea $X$ un espacio con un único punto no aislado $x$. Si $A \subset X$ no es cerrado entonces $c l_{X}(A)=A \cup\{x\}$.

2.11 Proposición ([21]). Si $X$ es un espacio de Hausdorff, secuencial y Whyburn, entonces es Fréchet-Urysohn.

Demostración. Sean $A \subseteq X$ y $x \in c l_{X}(A) \backslash A$. Por hipótesis, existe un subconjunto $B \subset A$ tal que $c l_{X}(B) \backslash A=\{x\}$. Como $X$ es secuencial y $c l_{X}(B) \cap A$ no es cerrado, existe una sucesión $S \subset c l_{X}(B) \cap A$ que converge a un punto $y \in c l_{X}\left(c l_{X}(B) \cap A\right) \backslash\left(c l_{X}(B) \cap A\right) \subset c l_{X}(B) \backslash A$. De hecho, como $X$ es de Hausdorff, resulta que $x=y$ y, en consecuencia, $S$ es una sucesión en $A$ que converge a $x$.

\subsection{Espacios de Whyburn bajo ciertos tipos de Funciones Continuas}

Es conocido que la radialidad se preserva bajo mapeos pseudoabiertos y mapeos cerrados; la pseudoradialidad se preserva bajo mapeos cocientes. Como es natural nos interesa saber si las propiedades de Whyburn y débilmente Whyburn se preservan por algunas de estas funciones. A continuación se ofrecen resultados al respecto.

2.12 Ejemplo ([16]). Sea $\mathfrak{c}=2^{\omega}$ un cardinal regular. Entonces existe una función continua y abierta de un espacio débilmente Whyburn sobre un espacio que no es débilmente Whyburn.

Demostración. Identificamos $\mathfrak{c}$ con el menor ordinal de cardinal $\mathfrak{c}$ y sea $I=$ $[0,1]$ en $\mathbb{R}$. Sea $\varphi: \mathfrak{c} \rightarrow I$ cualquier biyección. Denotamos por $Y$ a la unión disjunta de $\mathfrak{c}$ con $I$ y, consideremos en $Y$ la siguiente topología: cualquier punto $\alpha \in \mathfrak{c}$ es aislado en $Y$ y una vecindad básica $U$ de un punto $x \in I$ es de la forma $U=J \cup\left\{\alpha \in \mathfrak{c}: \alpha>\alpha_{0}, \varphi(\alpha) \in J\right\}$ donde $J$ es una vecindad abierta de $x$ en $I$ y $\alpha_{0} \in \mathfrak{c}$.

Claramente $\mathfrak{c}$ es denso en $Y$. Mostraremos que no existe $B \subseteq \mathfrak{c}$ tal que $\left|c l_{Y}(B) \backslash \mathfrak{c}\right|=1$. Supongamos que $B \subseteq \mathfrak{c}$ no es cerrado en $Y$. Puesto que $\mathfrak{c}$ 
es regular, por la definición de la topología en $Y, B$ es cofinal en c. Entonces $|B|=\mathfrak{c}$, lo que implica que $\varphi(B) \subseteq I$ también tiene cardinalidad $\mathfrak{c}$. Por la compacidad de $I$ existe un punto de acumulación completa $z$ en $\varphi(B)$. Demostraremos que $z \in c l_{Y}(B)$. Sea $U$ una vecindad de $z$ en $I$. Entonces $|U \cap \varphi(B)|=\mathfrak{c}$. Una vecindad de $z$ en $Y$ es de la forma $V=U \cup\{\alpha \in$ $\mathfrak{c}: \alpha>\alpha_{0}$ y $\left.\varphi(\alpha) \in U\right\}$ con $\alpha_{0} \in \mathfrak{c}$. Ahora bien, hay $\mathfrak{c}$ puntos $\alpha$ de $B$ tales que $\varphi(\alpha) \in U$. Así que para cualquier $\alpha_{0} \in \mathfrak{c}$ existe $\alpha>\alpha_{0}$ tal que $\alpha \in B$ y $\varphi(\alpha) \in U$. De manera que $V \cap B \neq \emptyset \mathrm{y}$, en consecuencia, $z \in c l_{Y}(B)$. Finalmente, demostraremos que si $D \subset I$ y $|D|=\mathfrak{c}$ entonces $D$ tiene al menos dos puntos de acumulación completa. Supongamos que $w$ es el único punto de acumulación completa de $D$. Entonces para cada $n \in \mathbb{N}$ se tiene que $\left|D \backslash\left(w-\frac{1}{n}, w+\frac{1}{n}\right)\right|<\mathfrak{c}$, de lo contrario, $D$ tendría otro punto de acumulación completa. Esto implica que, $D \backslash\{w\}=\bigcup\left\{D \backslash\left(w-\frac{1}{n}, w+\frac{1}{n}\right): n \in \mathbb{N}\right\}$ es la unión numerable de conjuntos de cardinalidad menor que $\mathfrak{c}$. De modo que, $\mathfrak{c}$ es la suma numerable de cardinales menores que $\mathfrak{c}$, por lo que $\mathfrak{c}$ tiene cofinalidad numerable. Esto es una contradicción. Por tanto, $\left|c l_{Y}(B) \backslash \mathfrak{c}\right|>1$.

Sea $X$ la unión disjunta del producto cartesiano $\mathfrak{c} \times I$ e $I$. Consideremos la siguiente topología en $X$. Cualquier punto $\langle\alpha, r\rangle \in \mathfrak{c} \times I$ es punto aislado en $X$. Sea $J$ cualquier vecindad de $x$ en $I$ y para cualquier $y \in J$ sean $\alpha_{y} \in \mathfrak{c}$ cualquier número ordinal y $J_{y}$ cualquier vecindad de $y$ en $J$; entonces denotamos a una vecindad básica $U$ de $x \in I$ como $U=J \cup \bigcup\{\langle\alpha, y\rangle: y \in$ $J, \alpha>\alpha_{y}$ y $\left.\varphi(\alpha) \in J_{y}\right\}$. Finalmente, sea $f: X \rightarrow Y$ la función definida por $f(\langle\alpha, r\rangle)=\alpha$ para cada $\langle\alpha, r\rangle \in \mathfrak{c} \times I$ y $f(x)=x$ para toda $x \in I$.

Afirmamos que $X$ es un espacio de Hausdorff y débilmente de Whyburn. En efecto, sea $E$ cualquier subconjunto no cerrado de $X$. Si $E \cap I$ es cerrado y $x \in c l_{X}(E) \backslash E \subseteq I$, escogemos una vecindad abierta $U$ de $x$ tal que $U \cap(E \cap I)=\emptyset$, entonces $x \in c_{X}(U \cap E)$ y reemplazamos $E$ por $E \cap U \subseteq \mathfrak{c} \times I$. Si $E \cap I$ no es cerrado y puesto que $I$ es primero numerable, elegimos una sucesión en $E \cap I$ que converja a un punto del complemento de $E$. Entonces podemos suponer que $E \cap I=\emptyset$. Supogamos primero que existe un elemento $y \in I$ tal que $y \in c_{X}\left(E_{y}\right)$ donde $E_{y}=\{\langle\alpha, y\rangle: \alpha \in \mathfrak{c}\} \cap E$. Entonces $y$ es el único punto de acumulación de $E_{y}$ y $c l_{X}\left(E_{y}\right) \backslash E_{y}=\{y\}$. Supongamos que ningún elemento $y \in I$ es punto de acumulación de $E_{y}$. Entonces para cada $y$ podemos encontrar una vecindad de la forma $V_{y}=J_{y} \cup \bigcup_{v \in J_{y}}\{\langle\alpha, v\rangle: \alpha\rangle$ $\left.\alpha(y)_{v}, \varphi(\alpha) \in L(y)_{v}\right\}$ donde $L(y)_{v}$ es una vecindad abierta de $v$ en $J_{y}$ y tanto $L(y)_{v}$ como $\alpha(y)_{v}$ dependen de $v$ y $y$, tal que $V_{y} \cap E_{y}=\emptyset$. En particular, si $\langle\alpha, y\rangle$ satisface $\alpha>\alpha(y)_{y}$ y $\varphi(\alpha) \in L(y)_{y}$, entonces $\langle\alpha, y\rangle \notin E$ porque 
$\langle\alpha, y\rangle \in V_{y}$. Sea $x \in I$. Considerando la vecindad abierta de $x$ definida por $V=I \cup \bigcup_{y \in I}\left\{\langle\alpha, y\rangle: \alpha>\alpha(y)_{y}, \varphi(\alpha) \in L(y)_{y}\right\}$, se sigue que $V \cap E=\emptyset$. De hecho, como $\langle\alpha, y\rangle \in V$ se tiene por definición que $\alpha>\alpha(y)_{y}$ y $\varphi(\alpha) \in L(y)_{y}$. Por tanto, $\langle\alpha, y\rangle \notin E$, lo que contradice que $E$ no es cerrado en $X$.

Sólo resta verificar que la función $f$ es continua y abierta. Sea $U^{\prime}=J \cup$ $\left\{\alpha \in \mathfrak{c}: \alpha>\alpha_{0}, \varphi(\alpha) \in J\right\}$ cualquier abierto básico de un punto $x \in I \subset Y$. Entonces $f^{-1}\left(U^{\prime}\right)=J \cup\left\{\langle\alpha, \beta\rangle \in \mathfrak{c} \times I: \alpha>\alpha_{0}, \varphi(\alpha) \in J\right.$ y $\left.\beta \in I\right\}$ es un abierto básico del punto $x \in X$. De modo que $f$ es continua. Ahora, sea $V^{\prime}=J \cup \bigcup_{y \in J}\left\{\langle\alpha, y\rangle: \alpha>\alpha_{y}\right.$ y $\left.\varphi(\alpha) \in J_{y}\right\}$ cualquier abierto básico de un punto $x \in I \subset X$, donde $J_{y}$ es una vecindad abierta de $y$ en $J$. Sea $y \in f\left(V^{\prime}\right)$. El conjunto $W=J_{y} \cup\left\{\alpha \in \mathfrak{c}: \alpha>\alpha_{y}\right.$ y $\left.\varphi(\alpha) \in J_{y}\right\}$ es un abierto básico de $y$ contenido en $f\left(V^{\prime}\right)$. De aquí, $f\left(V^{\prime}\right)$ es un conjunto abierto en $Y$. Así $f$ es abierta.

2.13 Ejemplo ([16]). Existe una función continua y abierta de un espacio de Whyburn sobre un espacio que no es de Whyburn.

Demostración. Para cualquier $\alpha<\omega_{1}$ sea $X_{\alpha}$ el espacio ordenado $[0, \alpha) \subset \omega_{1}$; consideremos la suma topológica $S$ de todos los espacios $X_{\alpha}$ para $\alpha<\omega_{1}$. Denotamos el punto $\gamma$ en el espacio $X_{\alpha}$ por $\langle\alpha, \gamma\rangle$. Sea $X=S \cup\{\infty\}$ donde $\infty \notin S$ con la siguiente topología: la topología en $X_{\alpha}$ es la topología del orden y para $\gamma<\rho<\omega_{1}$ una vecindad básica de $\infty$ es dada por $U_{\rho, \gamma}=$ $\{\infty\} \cup \bigcup_{\alpha>\rho}\{\langle\alpha, \beta\rangle: \beta \in(\gamma, \alpha)\}$.

Mostremos que el espacio $X$ es de Whyburn. Como para cualquier $\alpha$ el espacio $X_{\alpha}$ es de Hausdorff y primero numerable, sólo necesitamos verificar la propiedad en el punto $\infty$. Supongamos que $\infty \in c_{X}(E) \backslash E$ para algún $E \subseteq S$. Obsérvese que $E$ contiene puntos $\langle\alpha, \beta\rangle$ para una cantidad no numerable de $\alpha$ 's y $\beta$ 's arbitrariamente grandes en $X_{\alpha}$, es decir, para cualquier $\gamma<\omega_{1}$ existe un punto $\langle\alpha, \beta\rangle \in E$ tal que $\gamma<\beta<\alpha$. Construimos por inducción sobre $\omega_{1}$ un subconjunto $E^{\prime}$ de $E$ como sigue: sea $\alpha_{1} \in \omega_{1}$ tal que $E \cap$ $X_{\alpha_{1}} \neq \emptyset$ y escogemos $\left\langle\alpha_{1}, \beta_{1}\right\rangle \in E \cap X_{\alpha_{1}}$. Supongamos que tenemos definido $\left\langle\alpha_{\xi}, \beta_{\xi}\right\rangle \in E \cap X_{\alpha_{\xi}}$ para cada $\xi<\delta$, donde ambas sucesiones $\left\{\alpha_{\xi}\right\}_{\xi<\delta}$ y $\left\{\beta_{\xi}\right\}_{\xi<\delta}$ son crecientes. Sea $\sigma$ un ordinal más grande que el $\sup \left(\left\{\alpha_{\xi}: \xi<\delta\right\}\right)$ y que el $\sup \left(\left\{\beta_{\xi}: \xi<\delta\right\}\right)$. Dado que $U_{\sigma+1, \sigma} \cap E \neq \emptyset$, escogemos un punto $\left\langle\alpha_{\delta}, \beta_{\delta}\right\rangle \in E$ tal que $\alpha_{\delta}>\alpha_{\xi}$ y $\beta_{\delta}>\beta_{\xi}$ para cada $\xi<\delta$. El conjunto $E^{\prime}=\left\{\left\langle\alpha_{\delta}, \beta_{\delta}\right\rangle: \delta<\omega_{1}\right\}$ es casi cerrado y $E^{\prime} \rightarrow \infty$. En efecto, $\infty$ es punto límite de $E^{\prime}$ por construcción y, $E^{\prime}$ intersecta a cada $X_{\alpha}$ a lo más en un 
punto. De manera que ningún punto de $S$ es punto límite de $E^{\prime}$. Por tanto, $X$ es de Whyburn.

Por el Ejemplo 2.9 sabemos que el espacio $\omega_{1}+1$ no es de Whyburn. Consideremos la función $f: X \rightarrow \omega_{1}+1$ definida por $f(\langle\alpha, \beta\rangle)=\beta$ para todo $\langle\alpha, \beta\rangle \in S$ y $f(\infty)=\omega_{1}$. Para corroborar que la función $f$ es continua y abierta, basta notar que $f^{-1}\left(\left(\gamma, \omega_{1}\right]\right)=U_{\gamma+1, \gamma}$ es un conjunto abierto en $X$ y $f\left(U_{\rho, \gamma}\right)=\left(\gamma, \omega_{1}\right]$ es un conjunto abierto en $\omega_{1}+1$. Esto termina la prueba.

2.14 Proposición ([21]). Si $X$ es un espacio Whyburn y $f: X \longrightarrow Y$ es un mapeo suprayectivo y cerrado, entonces $Y$ es Whyburn.

Demostración. Supongamos que $A \subset Y$ y $y \in c l_{Y}(A) \backslash A$. Sea $B=f^{-1}(A)$. Nótese que $B$ no es cerrado en $X$ pues si lo fuera $A=f[B]$ sería cerrado en $Y$. Además, $f^{-1}(y) \cap c l_{X}(B) \neq \emptyset$. Por hipótesis, para cada $x \in c l_{X}(B) \backslash B$ existe un conjunto $C \subset B$ tal que $c l_{X}(C) \backslash B=\{x\}$. En particular, esto es cierto para cada $x \in f^{-1}(y)$. Luego, $f(C) \subset A$ y $c l_{Y}(f(C)) \backslash A \subset f\left(c l_{X}(C)\right) \backslash A \subset$ $f\left(c l_{X}(C) \backslash B\right)=f(\{x\}) \subset\{y\}$; además, $x \in c l_{X}(C)$ implica que $y=f(x) \in$ $f\left[c l_{X}(C)\right] \subseteq c l_{Y}(f(C))$, por lo que concluimos que $Y$ es Whyburn.

El resultado anterior también es válido para espacios débilmente Whyburn, como la prueba es muy similar la omitimos.

2.15 Proposición ([21]). La imagen cociente de un espacio Whyburn no es necesariamente un espacio Whyburn.

Demostración. Tomemos cualquier espacio secuencial $X$ que no sea FréchetUrysohn. Entonces $X$ es la imagen cociente de un espacio métrico $M([2])$. Como cualquier espacio secuencial y Whyburn es de Fréchet, por la Proposición 2.11, se puede concluir que la imagen cociente de un espacio métrico no necesariamente es Whyburn.

2.16 Proposición ([21]). Si X es un espacio de Hausdorff, infinito, débilmente Whyburn y compacto, entonces tiene una sucesión convergente no trivial. 
Demostración. Por el Lema 1.21, podemos encontrar un subconjunto $A=$ $\left\{x_{n}: n \in \omega\right\}$ infinito y discreto de $X$. Como $X$ es compacto se tiene que $A$ no es cerrado y puesto que $X$ es débilmente Whyburn, existe $B \subset A$ tal que $\left|c l_{X}(B) \backslash A\right|=1$. Entonces, $c l_{X}(B)=B \cup\{p\}$ para algún $p \in c l_{X}(A) \backslash A$ es compacto, por ser cerrado en $X$. Dado que $B$ es discreto, $B \cup\{p\}$ es una sucesión convergente. Esto es, $B$ converge a $p$.

2.17 Corolario ([21]). El espacio $\beta \omega \backslash \omega$ no es débilmente Whyburn.

2.18 Ejemplo. Existe un espacio de Hausdorff que no es débilmente Whyburn y puede expresarse como unión de dos subespacios primero numerables.

Demostración. Denotaremos por $\kappa \omega \backslash \omega$ a la familia de todos los ultrafiltros de abiertos en $\omega$ que no convergen a ningún punto de $\omega$. En el conjunto $\omega \cup(\kappa \omega \backslash \omega)$ introduciremos una topología $\tau$ de la siguiente manera: cada $x \in \omega$ es aislado y una vecindad de $\xi \in(\kappa \omega \backslash \omega)$ es un conjunto de la forma $\{\xi\} \cup U$, donde $U \in \xi$. El espacio $\kappa \omega=(\omega \cup(\kappa \omega \backslash \omega), \tau)$ se llama extensión de Katetov del espacio $\omega$.

Obsérvese que por construcción, $\omega$ y $\kappa \omega \backslash \omega$ al ser discretos son primero numerables. Además, $\kappa \omega$ y $\beta \omega$ son los mismos conjuntos y si $A \subseteq \omega$, entonces $c l_{\beta \omega}(A)$ y $c l_{\kappa \omega}(A)$ son iguales. Se sabe que si $A \subseteq \omega$ es infinito, entonces $c_{\beta \omega}(A)$ tiene cardinalidad $2^{\mathfrak{c}}$. Por tanto, $\kappa \omega$ no es débilmente Whyburn.

2.19 Proposición ([21]). Es independiente de ZFC que cualquier espacio compacto y de Hausdorff $X$ con estrechez numerable sea débilmente Whyburn.

Demostración. En ([5],Teorema 2.2) se muestra que existen modelos de ZFC en que cualquier espacio compacto de Hausdorff con estrechez numerable es secuencial y, por la Proposición 2.4, débilmente Whyburn. Por otro lado, bajo el principio $\diamond$ de Jensen, en ([12]) se construye un espacio compacto con estrechez numerable que no tiene sucesiones convergentes. Por la Proposición 2.16 este espacio no es débilmente Whyburn.

Por otro lado, en ZFC hay un espacio $H$-cerrado con estrechez numerable que no es débilmente Whyburn, a saber, el espacio $\kappa \omega$ visto en el Ejemplo 2.18 . 
2.20 Proposición ([8]). Si X es un espacio de Hausdorff y semiradial, entonces es débilmente Whyburn.

Demostración. Sea $A$ un subconjunto no cerrado de $X$. Si $\kappa$ es el mínimo cardinal tal que $A$ no es $\kappa$-cerrado, entonces existe una cadena $F=\left\{x_{\xi}: \xi \in\right.$ $\kappa\} \subset A$ que converge a un punto $x \in X \backslash A$. De modo que, para cada abierto $U \subset X$ que contiene a $x$, existe $\lambda<\kappa$ tal que $\left\{x_{\xi}: \xi>\lambda\right\} \subseteq U$. Como $X$ es un espacio de Hausdorff, si $y \in c l_{X}(F)$, existen vecindades $V$ de $y$ y $U$ de $x$ tales que $V \cap F \subseteq\left\{x_{\xi}: \xi \leq \lambda\right\}$ para alguna $\lambda<\kappa$. O sea $y$ está en la cerradura de un segmento inicial de $F$, el cual tiene cardinalidad menor que $\kappa$. Por la minimalidad de $\kappa$ se tiene que la cerradura de cualquier segmento inicial de $F$ está contenido en $A$, lo que muestra que $c l_{X}(F) \backslash A=\{x\}$. Así, $X$ es débilmente Whyburn.

2.21 Proposición ([8]). Si X es un espacio de Hausdorff, compacto y débilmente Whyburn entonces es pseudoradial.

Demostración. Sean $A \subset X$ y $x \in c l_{X}(A) \backslash A$. Entonces existe $B \subset A$ tal que $c l_{X}(B) \backslash A=\{x\}$. Por el Teorema 1.11, podemos elegir una base local $\left\{U_{\xi}: \xi \in \kappa\right\}$ de $x$ en $c l_{X}(B)$ de cardinalidad mínima que satisfaga que $\bigcap_{\xi \in \kappa} c l_{c l_{X}(B)}\left(U_{\xi}\right)=\{x\}$. Por minimalidad, si $\nu<\kappa$ entonces en $c l_{X}(B)$ se tiene que $\left[\bigcap_{\nu \in \xi} c l_{c l_{X}(B)}\left(U_{\nu}\right)\right] \backslash\{x\} \neq \emptyset$ para toda $\xi \in \kappa$. Para cualquier $\xi$ escogemos un punto $x_{\xi} \in\left(\left[\bigcap_{\nu \in \xi} c l_{c l_{X}(B)}\left(U_{\nu}\right)\right] \backslash\{x\}\right)$ de manera que $\left\{x_{\xi}: \xi \in\right.$ $\kappa\}$ forma una cadena en $c l_{X}(B) \backslash\{x\}=B$. Dada una vecindad $V$ de $x$, por regularidad de $c l_{X}(B)$, existe $\xi_{0} \in \kappa$ tal que $c l_{c l_{X}(B)}\left(U_{\xi_{0}}\right) \subseteq V$. Si $\lambda \geq \xi_{0}$, entonces $x_{\lambda} \in \bigcap_{\nu \leq \lambda} c l_{c l_{X}(B)}\left(U_{\nu}\right)$, lo que quiere decir que $x_{\lambda} \in c l_{c l_{X}(B)}\left(U_{\xi_{0}}\right) \subseteq$ $V$. Por construcción, la cadena $\left\{x_{\xi}: \xi \in \kappa\right\}$ converge a $x \in X \backslash A$. Por tanto, $X$ es pseudoradial.

Si aplicamos la prueba anterior a cada punto de $c l_{X}(A) \backslash A$, se tiene la siguiente:

2.22 Proposición ([8]). Cualquier espacio compacto, de Hausdorff y Whyburn es radial. 
2.23 Proposición ([8]). Cualquier espacio numerablemente compacto, de Hausdorff y débilmente Whyburn es secuencialmente compacto.

Demostración. Sean $X$ como en la hipótesis del enunciado y $A=\left\{x_{1}, x_{2}, \ldots\right\}$ una sucesión infinita de puntos de $X$. Entonces $A$ tiene un punto de acumulación $x$. Podemos suponer que $x \notin A$ y, por el Lema 1.21, que $A$ es discreto. Además como $A$ no es cerrado, existe una subsucesión $B \subseteq A$ con un sólo punto de acumulación $p$. Esto es, $c l_{X}(B)=B \cup\{p\}$ es numerablemente compacto y numerable, por consiguiente, compacto. Puesto que $B$ es discreto, $B \cup\{p\}$ es una sucesión convergente, o sea, $B$ converge a $p$.

2.24 Teorema ([21]). Sea $X$ un espacio de Hausdorff, numerablemente compacto y de Whyburn. Entonces $X$ es Fréchet-Urysohn.

Demostración. Supongamos que $A \subset X$ y $x \in c_{X}(A) \backslash A$. Como $X$ es de Whyburn, existe un subconjunto infinito $P \subset A$ tal que $c l_{X}(P) \backslash A=\{x\}$. Por el Lema 1.24, existe una familia maximal celular $\gamma$ de subconjuntos abiertos de $P$ cuyas cerraduras no contienen a $x$. Luego, $\bigcup \gamma$ es densa en $P$ y debido a que $x \in c l_{X}(P)$ se tiene que $x \in c l_{X}(\bigcup \gamma)$. Considerando que $P$ es de Whyburn, por la Proposición 2.5, existe $Q \subset \bigcup \gamma$ tal que $c l_{X}(Q) \backslash \bigcup \gamma=\{x\}$. Sea $\gamma^{\prime}=\{U \in \gamma: U \cap Q \neq \emptyset\}$. Supongamos que $\gamma^{\prime}$ es finito, digamos $\gamma^{\prime}=$ $\left\{U_{1}, U_{2}, \ldots, U_{n}: U_{i} \in \gamma\right.$ para cada $\left.i\right\}$. De modo que, $Q \subset \bigcup_{i=1}^{n} U_{i}$ y, como se tiene una unión finita, $c l_{X}(Q) \subseteq c l_{X}\left(\bigcup_{i=1}^{n} U_{i}\right)=\bigcup_{i=1}^{n} c l_{X}\left(U_{i}\right)$. No obstante, $x \notin \bigcup_{i=1}^{n} c l_{X}\left(U_{i}\right)$ y, por tanto, $x \notin c l_{X}(Q)$, se tiene una contradicción. Por tanto, $\gamma^{\prime}$ es infinito.

Para $U \in \gamma^{\prime}$ tomamos $x_{u} \in U \cap Q$. El conjunto $B=\left\{x_{u}: U \in \gamma^{\prime}\right\}$ es discreto infinito, por tanto no cerrado pues $X$ es numerablemente compacto. Además, $\left(c l_{X}(B) \backslash B\right) \cap \bigcup \gamma=\emptyset$, pues de lo contrario existe $U^{\prime} \in \gamma$ tal que contiene puntos distintos de $x_{u^{\prime}}$, lo que no puede suceder porque el conjunto $B$ es discreto. Luego, como $c l_{X}(Q)=Q \cup\{x\}$ se sigue que $c l_{X}(B) \subseteq Q \cup\{x\}$ y, por ([11], Teorema 3.10.4), $Q \cup\{x\}$ es numerablemente compacto. De aquí $B \cup\{x\}$ es un espacio infinito numerablemente compacto y, dado que $B$ es discreto, tiene un sólo punto no aislado $x$. Por tanto, $B \cup\{x\}$ es compacto y cualquier subconjunto numerable infinito de $B$ es una sucesión que converge a $x$. Esto termina la prueba.

Un espacio de Hausdorff $X$ se llama espacio $k$, si $A \subset X$ es cerrado cuando $A \cap K$ es cerrado para cualquier subespacio compacto $K$ del espacio $X$. 
2.25 Corolario ([21]). Si X es un espacio $k$ que es de Whyburn, entonces es Fréchet-Urysohn.

Demostración. Sea $A \subset X$ y $x \in c_{X}(A) \backslash A$. Como $X$ es de Whyburn, existe un conjunto casi cerrado $F \subset A$ tal que $c l_{X}(F) \backslash A=\{x\}$. Dado que $F$ no es cerrado, existe un compacto $K \subset X$ tal que $K \cap F$ no es cerrado y, por tanto, $c l_{X}(K \cap F) \backslash F=\{x\}$. Luego, $K$ es numerablemente compacto y, por la Proposición 2.5, es de Whyburn. Por tanto, por el Teorema 2.24, $K$ es Fréchet-Urysohn. De esta manera existe una sucesión $S \subset K \cap F$ que a su vez está contenida en $A$ y que converge a $x$.

2.26 Definición. Un espacio $X$ es disperso, siempre que cualquier subespacio no vacío $Y \subset X$ tiene un punto aislado (con respecto a la topología relativa).

2.27 Teorema ([21]). Cualquier espacio $T_{3} y$ disperso es débilmente Whyburn.

Demostración. Sea $X$ un espacio disperso. Para cualquier subconjunto $Y \subset$ $X$ denotamos por $i(Y)$ al conjunto de puntos aislados del subespacio $Y$. Supongamos que un subconjunto no cerrado $A \subset X$ es Whyburn cerrado.

Sean $A_{0}=i(A)$ y $B_{0}=c l_{X}\left(A_{0}\right) \backslash A_{0}$. Entonces, $A_{0}$ es denso en $A$, pues de lo contrario $A \backslash c l_{X}\left(A_{0}\right)$ sería un abierto no vacío que no contiene puntos aislados de $A$; además, $B_{0}$ es cerrado en $X$. Si tenemos conjuntos $A_{\alpha}$ y $B_{\alpha}$, sean $A_{\alpha+1}=i\left(B_{\alpha}\right)$ y $B_{\alpha+1}=B_{\alpha} \backslash A_{\alpha+1}$. Si $\beta$ es un ordinal límite y tenemos $A_{\alpha}, B_{\alpha}$ para cada $\alpha<\beta$, definimos $A_{\beta}=\emptyset$ y $B_{\beta}=\bigcap\left\{B_{\alpha}: \alpha<\beta\right\}$.

Como $X$ es disperso, existe un ordinal $\gamma$ tal que $c l_{X}\left(A_{0}\right)=c l_{X}(A)=$ $\bigcup\left\{A_{\alpha}: \alpha<\gamma\right\}$. Sea $\beta=\min \left\{\alpha<\gamma: A_{\alpha} \cap\left(c l_{X}(A) \backslash A\right) \neq \emptyset\right\}$. Entonces, $\beta$ no es un ordinal límite, es decir, $\beta$ es un ordinal sucesor $\beta=\beta_{0}+1$.

Escojamos un punto $x \in A_{\beta} \backslash A=i\left(B_{\beta-1}\right) \backslash A=i\left(B_{\beta_{0}}\right) \backslash A$. Como $x$ es un punto aislado de $B_{\beta_{0}}$, existe un abierto $U \subset X$ tal que $U \cap B_{\beta_{0}}=\{x\}$. Tomemos cualquier abierto $V \subset X$ tal que $x \in V \subset c l_{X}(V) \subset U$, por regularidad de $X$, y consideremos el conjunto $F=\left(c l_{X}(V) \cap A\right) \backslash\{x\}$. Puesto que $x \in c l_{X}(A) \backslash A$ se sigue que $x \in c l_{X}(F)$. Como $F \subset A$ se sigue que, $c l_{X}(F) \subset c l_{X}(A)=\bigcup\left\{A_{\alpha}: \alpha \leq \beta_{0}\right\} \cup B_{\beta_{0}}$ y, dado que, $c l_{X}(F) \cap B_{\beta_{0}} \subset$ $c l_{X}(V) \cap B_{\beta_{0}} \subset U \cap B_{\beta_{0}}=\{x\}$, resulta que $c l_{X}(F) \cap B_{\beta_{0}}=\{x\}$. Por tanto, $c l_{X}(F) \backslash\{x\} \subset \bigcup\left\{A_{\alpha}: \alpha \leq \beta_{0}\right\} \subset A$. De aquí $c l_{X}\left(c l_{X}(F) \backslash\{x\}\right) \backslash A=\{x\}$, lo que termina la prueba. 
El Teorema anterior es falso si se sustituye Hausdorff en el lugar de $T_{3}$, como lo muestra el espacio $\kappa \omega$ visto en el Ejemplo 2.18.

2.28 Corolario ([21]). Si X es un espacio compacto de Hausdorff y disperso, entonces $X$ es pseudoradial.

Demostración. Por el Teorema 2.27, $X$ es débilmente Whyburn y, por la Proposición 2.21, es pseudoradial.

Ser disperso es una propiedad hereditaria y, por lo tanto, se tiene el siguiente:

2.29 Corolario ([21]). Cualquier espacio disperso y $T_{3}$, es hereditariamente débilmente Whyburn.

2.30 Teorema ([7]). Si $X$ es un espacio de Hausdorff, Whyburn y pseudoradial, entonces es radial.

Demostración. Sean $A \subset X$ y $x \in c_{X}(A) \backslash A$. Como $X$ es Whyburn, existe un subconjunto $B \subset A$ tal que $c l_{X}(B) \backslash A=\{x\}$ y, como $X$ es pseudoradial, existe una sucesión transfinita en $c l_{X}(B) \backslash\{x\}$ que converge a $x$. Nótese que esta sucesión está en $A$. Por tanto, $X$ es radial.

2.31 Definición. Un espacio $X$ denso en si mismo es submaximal si cada subconjunto denso de $X$ es abierto en $X$ o equivalentemente, si cualquier subconjunto con interior vacío es cerrado y discreto.

2.32 Proposición ([7]). Si $X$ es un espacio $T_{3}$ y submaximal es de Whyburn.

Demostración. Sean $A \subset X$ y $x \in c l_{X}(A) \backslash A$. Entonces, $x \in c l_{X}\left(A \backslash \operatorname{int}_{X}(A)\right)$ o bien $x \in \operatorname{cl}_{X}\left(\operatorname{int}_{X}(A)\right)$, pero debido a que $\operatorname{int}_{X}\left(A \backslash i n t_{X}(A)\right)=\emptyset$ se sigue que $x \in c l_{X}\left(i n t_{X}(A)\right)$. Como $c l_{X}\left(i n t_{X}(A)\right) \backslash A$ es cerrado y discreto en $X$, existe una vecindad $W$ de $x$ tal que $W \cap\left[c l_{X}\left(i n t_{X}(A)\right) \backslash A\right]=\{x\}$. Por regularidad podemos suponer que $W$ es cerrado. Sea $B=W \cap i n t_{X}(A)$. Entonces, como $B \subset W$ y $B \subset \operatorname{int}_{X}(A)$, se sigue que $c l_{X}(B) \subset c l_{X}(W) \cap c l_{X}\left(i n t_{X}(A)\right)$ y, en tal caso, $c l_{X}(B) \backslash A \subset\left[c l_{X}(W) \cap c l_{X}\left(i n t_{X}(A)\right)\right] \backslash A=\{x\}$. Por tanto, $X$ es de Whyburn.

Un contraejemplo para espacios de Hausdorff es el espacio $\kappa \omega$, ya que todo subconjunto denso de $\kappa \omega$ es abierto en $\kappa \omega$. Sin embargo, por 2.18 este espacio ni siquiera es débilmente Whyburn. 


\subsection{Espacios Pseudocompactos de Whyburn}

En la presente sección, todos los espacios a considerar son de Hausdorff. Mostraremos que no se puede sustituir pseudocompacto en lugar de numerablemente compacto en el Teorema 2.24.

2.33 Teorema ([18]). Si la igualdad $\mathfrak{b}=\mathfrak{d}$ es válida, entonces existe un espacio de Tychonoff, disperso, separable, pseudocompacto y Whyburn de estrechez no numerable; por tanto no es Fréchet-Urysohn.

Demostración. Sea $\Phi=\left\{\varphi_{\alpha}: \alpha<\mathfrak{b}\right\}$ una familia dominante en $\left(\omega^{\omega},<^{*}\right)$. Por el Lema 1.34, podemos suponer que $\Phi$ está bien ordenado por $<^{*}$, así que $\alpha \rightarrow \varphi_{\alpha}$ es un isomorfismo de orden y, en particular, $\varphi_{\alpha}<^{*} \varphi_{\beta}$ para cada $\alpha<\beta<\mathfrak{b}$. Para el conjunto $C=\omega \times \omega$, sea $C_{n}=\{n\} \times \omega$ para cada $n \in \omega$. Dado $\varphi_{\alpha} \in \Phi$, definimos conjuntos $D_{\alpha}=\left\{(m, n) \in C: n \leq \varphi_{\alpha}(m)\right\}$ y $U_{\alpha}=C \backslash D_{\alpha}$. Al conjunto $S \subset C$ le llamamos selector si $\left|S \cap C_{n}\right| \leq 1$ para cualquier $n \in \omega$. Por recursión transfinita en $\alpha<\mathfrak{b}$ definimos una familia casi ajena $\mathcal{A}$ en el conjunto $C$ (ver 1.28), con las siguientes propiedades:

(1) $\mathcal{A}=\bigcup\left\{\mathcal{A}_{\alpha}: \alpha<\mathfrak{b}\right\}$ donde $\mathcal{A}$ consiste de selectores.

(2) La familia $\mathcal{A} \cup\left\{C_{n}: n \in \omega\right\}$ es maximal casi ajena.

(3) $A \subset D_{\alpha}$ y $A \subset^{*} U_{\beta}$ cuando $\beta<\alpha<\mathfrak{b}$ y $A \in \mathcal{A}_{\alpha}$.

Para $\alpha=0$ elegimos una familia maximal casi ajena $\mathcal{A}_{0}$ de selectores contenidos en $D_{0}$. Supongamos que hemos definido las familias $\mathcal{A}_{\beta}$ para cada $\beta<\alpha<\mathfrak{b}$.

Sea $\alpha=\delta+1$. Sabemos que $\varphi_{\delta}<^{*} \varphi_{\delta+1}$, es decir, $\left\{m: \varphi_{\delta+1}(m)>\varphi_{\delta}(m)\right\}$ es infinito. Entonces $D_{\alpha} \cap U_{\delta}$ es infinito, de modo que podemos elegir una familia maximal casi ajena $\mathcal{A}_{\alpha}$ donde cada $A \in \mathcal{A}_{\alpha}$ es un selector en $D_{\alpha} \cap U_{\delta}$.

Sea $\alpha$ un ordinal límite. Escogemos una familia $\mathcal{A}_{\alpha}$ constituida por selectores tal que

(4) Cualquier $A \in \mathcal{A}_{\alpha}$ es un subconjunto de $D_{\alpha}$.

(5) $\mathcal{A}_{\alpha}$ es casi ajena y el conjunto $A \cap D_{\beta}$ es finito para cualquier $\beta<\alpha$ y $A \in \mathcal{A}_{\alpha}$.

(6) $\mathcal{A}_{\alpha}$ es maximal con respecto a (4) y (5). 
La familia $\mathcal{A}=\bigcup\left\{A_{\alpha}: \alpha<\mathfrak{b}\right\}$ satisface (1) por construcción. Para corroborar (2), tomemos $A, B \in \mathcal{A}$ con $A \neq B$ entonces $A \in \mathcal{A}_{\alpha}$ y $B \in \mathcal{A}_{\beta}$. Supongamos sin pérdida de generalidad que $\alpha \leq \beta$. Si $\alpha=\beta$ entonces $A \cap B$ es finito porque $\mathcal{A}_{\alpha}$ es casi ajena y si $\alpha<\beta$, entonces $A \subset D_{\alpha}$ y $B \cap D_{\alpha}$ es finito, de manera que $A \cap B$ es finito. Puesto que $\mathcal{A}$ consiste de selectores, la familia $\mathcal{A} \cup\left\{C_{n}: n \in \omega\right\}$ sigue siendo casi ajena.

Veamos que $\mathcal{A}^{\prime}=\mathcal{A} \cup\left\{C_{n}: n \in \omega\right\}$ es maximal. Para tal efecto, tomemos un conjunto infinito $D \subset C$ tal que $D \cap A$ es finito para cualquier $A \in \mathcal{A}^{\prime}$. Como $D \cap C_{n}$ es finito para cada $n, D$ intersecta a un número infinito de $C_{n}$, lo que implica que $D$ contiene a un selector infinito $D^{\prime}$. Sin pérdida de generalidad, podemos suponer que $D=D^{\prime}$, es decir, $D$ es un selector. Como la familia $\Phi$ es dominante podemos extender $D$ a una función $\varphi: \omega \rightarrow \omega$ tal que existe $\gamma<\mathfrak{b}$ donde $\varphi<^{*} \varphi_{\gamma}$, lo que quiere decir que $D \subset^{*} D_{\gamma}$ para alguna $\gamma<\mathfrak{b}$. Por tanto, el conjunto $D \cap D_{\gamma}$ es infinito. Denotamos por $\alpha=\operatorname{mín}\left\{\delta<\mathfrak{b}: D \cap D_{\delta}\right.$ es infinito $\}$.

Si $\alpha=\eta+1$ entonces existe algún conjunto infinito $E \subset D$ que está en $D_{\alpha} \cap U_{\eta}$. Como $E$ es un selector y $\mathcal{A}_{\alpha}$ es una familia maximal de selectores en $D_{\alpha} \cap U_{\eta}$, el conjunto $E$ tiene intersección infinita con algún $A \in \mathcal{A}_{\alpha}$, lo que es una contradicción.

Si $\alpha$ es un ordinal límite, $H=D \cap D_{\alpha}$ es infinito. No obstante, $D_{\beta} \cap D$ es finito para cada $\beta<\alpha$, lo que implica que $D_{\beta} \cap H$ es finito para todo $\beta<\alpha$. De aquí $H \subset D$ y, en consecuencia, $H$ es selector en $D_{\alpha}$ que satisface (4) y (5) para elementos de $\mathcal{A}_{\alpha}$. Por (6), el conjunto $H \cap A$ es infinito para algún $A \in \mathcal{A}_{\alpha}$ lo que genera la contradicción final.

Para cada $A \in \mathcal{A}$ tomamos un punto cualquiera $p_{A} \notin C$ y un punto $p \notin C \cup\left\{p_{A}: A \in \mathcal{A}\right\}$; entonces $X=\{p\} \cup\left\{p_{A}: A \in \mathcal{A}\right\} \cup C$ es el conjunto fundamental del espacio requerido. Todos los puntos de $C$ son aislados en $X$ y la base de vecindades de cualquier $p_{A}$ es la familia $\left\{\left\{p_{A}\right\} \cup(A \backslash F)\right.$ : $F$ es un subconjunto finito de $A\}$. Finalmente, una base de $p$ está formada por los conjuntos $O_{\alpha}=\{p\} \cup U_{\alpha} \cup\left\{p_{A}: A \in \bigcup_{\beta>\alpha} \mathcal{A}_{\beta}\right\}$, donde $\alpha<\mathfrak{b}$.

El espacio $X$ es de Tychonoff porque es cero-dimensional por construcción; es disperso porque los espacios $C$ y $\left\{p_{A}: A \in \mathcal{A}\right\}$ son discretos; como $C$ es un subconjunto denso numerable de $X$, es separable. Ahora, para verificar que $X$ es pseudocompacto, puesto que $C$ consiste de puntos aislados, basta verificar que cualquier conjunto infinito $D \subset C$ tiene un punto de acumulación en $X$. Como la familia $\mathcal{A}^{\prime}=\mathcal{A} \cup\left\{C_{n}: n \in \omega\right\}$ es maximal, el conjunto $A \cap D$ es infinito para alguna $A \in \mathcal{A}^{\prime}$. Si $A=C_{n}$ para alguna $n \in \omega$, entonces 
$C_{n} \cap D$ es infinito y, por la definición de las vecindades de $p$, se sigue que $p \in c l_{X}(D) \backslash D$. Si no, entonces $A \in \mathcal{A}$. Como $A \cap D$ es infinito y, por definición de las vecindades de $p_{A}$, se tiene que $p_{A}$ es el punto de acumulación de $D$. Por tanto, $X$ es pseudocompacto.

Para ver que $X$ es Whyburn, tomemos cualquier $D \subset X$ y cualquier $x \in c l_{X}(D) \backslash D$, donde $x \in\{p\} \cup\left\{p_{A}: A \in \mathcal{A}\right\}$. Tenemos dos casos:

Caso 1. Si $x=p_{A}$ para algún $A \in \mathcal{A}$, entonces $\left\{p_{A}\right\} \cup A$ es una vecindad de $p_{A}$. Como $x \in c l_{X}(D \cap A)$ resulta que $\left(c l_{X}(D \cap A)\right) \backslash A=\{x\}$.

Caso 2. Si $x=p$ entonces $x \in c l_{X}(D \cap C)$ o $x \notin c l_{X}(D \cap C)$. Si $x \in$ $c l_{X}(D \cap C)$, supongamos que $D$ está formado por una cantidad finita de elementos de $C$ en cada columna $C_{n}$. Entonces, existe una función $\varphi_{\alpha}$ que domina a $D \cap C$ por lo cual su complemento es una vecindad de $x$ que no intersecta a $D$. De aquí $D \cap C_{n} \rightarrow x$ para algún $n \in \omega$. Si $x \notin c l_{X}(D \cap C)$, entonces $x \in c l_{X}\left(D \cap\left\{p_{A}: A \in \mathcal{A}\right\}\right)$. No obstante, el único punto no aislado en $c l_{X}\left(D \cap\left\{p_{A}: A \in \mathcal{A}\right\}\right)$ es $x$. Así, $D \cap\left\{p_{A}\right.$ : $A \in \mathcal{A}\}$ converge a $x$.

Por tanto $X$ es Whyburn. Finalmente, por definición de vecindad de $p$ se tiene que $p \in c l_{X}\left(\left\{p_{A}: A \in \mathcal{A}\right\}\right)$. Además, para cualquier subconjunto numerable $D \subset\left\{p_{A}: A \in \mathcal{A}\right\}$, puesto que $\mathfrak{b}$ tiene cofinalidad no numerable, existe $\alpha_{0} \in \mathfrak{b}$ tal que si $p_{A} \in D$, entonces $A \in \mathcal{A}_{\alpha}$ para algún $\alpha<\alpha_{0}$. De manera que $O_{\alpha_{0}} \cap$ $D=\emptyset$, es decir, $p \notin c l_{X}(D)$. Por eso $X$ tiene estrechez no numerable.

La prueba del siguiente Teorema, necesita de una construcción auxiliar para un conjunto infinito numerable $D$ que será identificado con el espacio discreto cuyo conjunto sea $D$. Denotaremos con $K$ al conjunto de Cantor, las funciones $\pi_{D}: D \times K \rightarrow D$ y $\pi_{K}: D \times K \rightarrow K$ representan las proyecciones naturales.

Si $A \subset D \times K$, entonces $A(d)$ denota al conjunto $\pi_{K}(A \cap(\{d\} \times K))$ para cualquier $d \in D$. Un conjunto $A \subset D \times K$ es llamado admisible si tiene las siguientes propiedades:

1. El conjunto $\pi_{D}(A)$ es infinito.

2. El conjunto $A(d)$ es abierto y cerrado en $K$ para cualquier $d \in \pi_{D}(A)$.

3. La familia $\left\{A(d): d \in \pi_{D}(A)\right\}$ es disjunta. 
Una familia $\mathcal{A}$ de subconjuntos admisibles es esencialmente disjunta si, para cualesquiera $A, B \in \mathcal{A}$ distintos, el conjunto $\{d \in D: A(d) \cap B(d) \neq \emptyset\}$ es finito.

Nótese que se puede construir una familia maximal esencialmente disjunta de subconjuntos admisibles de $D \times K$. En efecto, sea $\Gamma=\{\mathcal{A}$ : $\mathcal{A}$ es una familia esencialmente disjunta de subconjuntos admisibles de $D \times$ $K\}$. Dadas $\mathcal{A}, \mathcal{A}^{\prime} \in \Gamma$ hacemos $\mathcal{A} \leq \mathcal{A}^{\prime}$ si $\mathcal{A} \subset \mathcal{A}^{\prime}$. Esto hace que el conjunto $\Gamma$ sea parcialmente ordenado. Demostremos que todas las cadenas de $(\Gamma, \leq)$ son acotadas.

Sea $\mathcal{C}$ una cadena de $\Gamma$. Si $\mathcal{A}=\bigcup \mathcal{C}$, entonces para cualquier $\mathcal{A}^{\prime} \in \mathcal{C}$ se tiene que $\mathcal{A}^{\prime} \leq \mathcal{A}$. La cadena $\mathcal{C}$ será acotada por $\mathcal{A}$ si probamos que $\mathcal{A} \in \Gamma$, o sea, si probamos que $\mathcal{A}$ es una familia esencialmente disjunta de subconjuntos admisibles.

Tomemos conjuntos distintos $A, B \in \mathcal{A}$. Existen $\mathcal{A}_{A}, \mathcal{A}_{B} \in \mathcal{C}$, tales que $A \in \mathcal{A}_{A}$ y $B \in \mathcal{A}_{B}$. Como $\mathcal{C}$ es cadena, tenemos que $\mathcal{A}_{A} \subset \mathcal{A}_{B}$ ó $\mathcal{A}_{B} \subset \mathcal{A}_{A}$. Si $\mathcal{A}_{A} \subset \mathcal{A}_{B}$, entonces $A, B \in \mathcal{A}_{B}$ y por eso el conjunto $\{d \in D: A(d) \cap B(d) \neq \emptyset\}$ es finito. Lo mismo se tiene si $A, B \in \mathcal{A}_{A}$. Con esto queda establecido que $\Gamma$ tiene cadenas acotadas. Por el Lema de Zorn, existe un elemento maximal $\mathcal{A}(D) \in \Gamma$.

Finalmente, se puede definir el espacio auxiliar $P(D)=(D \times K) \cup\left\{p_{A}\right.$ : $A \in \mathcal{A}(D)\}$. Las bases locales de los puntos de $D \times K$ están dadas por sus bases locales usuales en $D \times K$ (donde, $D$ es discreto). Dado un $A \in \mathcal{A}(D)$ una base local en el punto $p_{A}$ es la familia $\left\{\left\{p_{A}\right\} \cup(A \backslash(F \times K)): F \subset D\right.$ es finito $\}$.

2.34 Lema ([18]). El espacio $P(D)$ es de Tychonoff, primero numerable, pseudocompacto y cero-dimensional; $D \times K$ es un subespacio de $P(D)$ y el conjunto $\left\{p_{A}: A \in \mathcal{A}(D)\right\}$ es cerrado y discreto.

Demostración. El espacio $P(D)$ es de Hausdorff debido a que $\mathcal{A}(D)$ es esencialmente disjunta; es cero-dimensional porque $U \cap(D \times K)$ es abierto y cerrado en $D \times K$ para cualquier vecindad básica $U$ del punto $p_{A}$, de manera que $P(D)$ es de Tyhonoff. Además, dado que las bases locales de los puntos $p_{A}$ son numerables y puesto que $D \times K$ es primero numerable, se tiene que $P(D)$ es primero numerable.

Para establecer que $P(D)$ es pseudocompacto, tomemos cualquier familia infinita $\mathcal{U}$ de subconjuntos abiertos, cerrados y no vacíos de $D \times K$. Veamos 
que existe un punto $x \in P(D)$ tal que cualquier vecindad de $x$ intersecta a una cantidad infinita de elementos de $\mathcal{U}$.

Si $\pi_{D}(\bigcup \mathcal{U})$ es finito, entonces $\pi_{D}(\bigcup \mathcal{U}) \subset G$ para algún subconjunto finito $G$ de $D$. De hecho, $G \times K$ es un compacto que contiene a cada $U_{n} \in \mathcal{U}$, de modo que $\mathcal{U}$ tiene un punto de acumulación.

Si $\pi_{D}(\bigcup \mathcal{U})$ es infinito, elegimos $d_{1} \in \pi_{D}\left(U_{1}\right)$ de manera que $\bigcup_{n>1} U_{n} \nsubseteq$ $\left\{d_{1}\right\} \times K$. Luego, existe $n_{2} \in \omega$ tal que $\pi_{D}\left(U_{n_{2}}\right) \nsubseteq\left\{d_{1}\right\}$. Ahora, escoge$\operatorname{mos} d_{2} \in \pi_{D}\left(U_{n_{2}}\right)$ tal que $\bigcup_{n>n_{2}} U_{n} \nsubseteq\left\{d_{1}, d_{2}\right\} \times K$. Asimismo, exis-te $n_{3} \in \omega$ tal que $\pi_{D}\left(U_{n_{3}}\right) \nsubseteq\left\{d_{1}, d_{2}\right\}$. Ahora elegimos $d_{3} \in \pi_{D}\left(U_{n_{3}}\right)$. Sean $V_{1}=U_{1} \cap\left(\left\{d_{1}\right\} \times K\right)$ y $V_{m}=U_{n_{m}} \cap\left(\left\{d_{m}\right\} \times K\right)$ para $m>1$. Denotamos por $V=\bigcup_{i=1}^{\infty} V_{i}$. Para construir un conjunto admisible, consideramos $\pi_{D}(V)=\left\{d_{n}: n \in \omega\right\}$ con $d_{n}<d_{n+1}$ para todo $n \in \omega$ y sea $\mathcal{V}=\left\{\pi_{K}\left(V_{n}\right): n \in \omega\right\}$. Supongamos que $\mathcal{V}$ no es disjunta, digamos que para un número infinito de $d_{n}$ 's se tiene que: (a) $\pi_{K}\left(V_{n}\right) \nsubseteq V_{11}$ o bien (b) $\pi_{K}\left(V_{n}\right) \nsubseteq V_{12}$. Sin perder generalidad, si ocurre (a) ponemos $A_{1}=V_{11}$. Pasamos al primer $d_{n}$ tal que para una cantidad infinita de $d_{n}$ 's sucede que $\pi_{K}\left(V_{n}\right) \nsubseteq V_{11}$, llamémosle $d_{n_{2}}$. Ahora a $V_{d_{n_{2}}}$ lo dividimos en dos subconjuntos abiertos y cerrados, $V_{d_{n_{2}}}^{1}$ y $V_{d_{n_{2}}}^{2}$. Entonces, para una infinidad de $d_{n}$ 's sucede que (c) $\pi_{K}\left(V_{d_{n}}\right) \nsubseteq \pi_{K}\left(V_{d_{n_{2}}}^{1}\right)$ o bien (d) $\pi_{K}\left(V_{d_{n}}\right) \nsubseteq \pi_{K}\left(V_{d_{n_{2}}}^{2}\right)$. Si ocurre (c) (respectivamente $(\mathrm{d})$ ), entonces ponemos $A_{2}=V_{d_{n_{2}}}^{1}$ (respectivamente $V_{d_{n_{2}}}^{2}$ ). Luego, al primer $d_{n}>d_{n_{2}}$ tal que $\pi_{K}\left(V_{d_{n}}\right) \nsubseteq \pi_{D}\left(V_{d_{n_{2}}}^{1}\right)$ para una infinidad de $d_{n}$ 's, lo llamamos $d_{n_{3}}$. Similarmente, escogemos $A_{3}=V_{d_{n_{3}}}^{1}$ (respectivamente $\left.V_{d_{n_{3}}}^{2}\right)$ y, procediendo inductivamente, se tiene que $A=\bigcup_{n \in \omega} A_{n}$ es admisible. Por la maximalidad de la familia $\mathcal{A}(D)$ existe $B \in \mathcal{A}(D)$ tal que $A(d) \cap B(d) \neq \emptyset$ para una infinidad de $d$ 's. Luego, como cada vecindad del punto $p_{B}$ intersecta a una infinidad de elementos de $\mathcal{U}$, podemos concluir que $P(D)$ es pseudocompacto.

2.35 Teorema ([18]). Existe (en ZFC) un espacio de Tychonoff, pseudocompacto (no separable), disperso y Whyburn de estrechez no numerable; por tanto, no es Fréchet-Urysohn.

Demostración. Se denota por $L$ al subespacio del ordinal $\mathfrak{c}^{+}$que consiste de los ordinales límite de cofinalidad numerable. Al darle a $L$ la topología del orden es natural decir que un conjunto infinito numerable $S \subset L$ es una sucesión que converge a $\alpha \in L$ si $S \subset \alpha, \sup (S)=\alpha$ y $S \cap \beta$ es finito para cualquier $\beta<\alpha$. 
Si $\alpha$ no es ordinal límite, es decir, si $\alpha \in L$ y $\alpha=\beta+\omega$ para algún $\beta<\mathfrak{c}^{+}$, entonces denotamos $\mathcal{D}_{\alpha}=\{[\beta, \alpha)\}$ donde $[\beta, \alpha)=\{\gamma: \beta \leq \gamma<\alpha\}$. Si $\alpha$ es un punto límite en $L$, definimos $\mathcal{D}_{\alpha}$ como una familia maximal casi ajena de sucesiones que convergen a $\alpha$. Ahora, probaremos que $\mathcal{D}=\bigcup\left\{\mathcal{D}_{\alpha}: \alpha \in L\right\}$ es una familia maximal casi ajena de subconjuntos numerables de $\mathfrak{c}^{+}$.

Primero debemos mostrar que $\mathcal{D}$ es una familia casi ajena. Sean $D \in \mathcal{D}_{\alpha_{1}}$ y $E \in \mathcal{D}_{\alpha_{2}}$. Entonces, consideremos los siguientes casos:

a) Si $\alpha_{1}$ y $\alpha_{2}$ son aislados en $L$ entonces $D \cap E=\emptyset$.

b) Si $\alpha_{1}$ es aislado y $\alpha_{2}$ es límite con $\alpha_{1}<\alpha_{2}$, entonces $D \cap E$ es finito.

c) Si $\alpha_{1}$ es aislado y $\alpha_{2}$ es límite con $\alpha_{2}<\alpha_{1}$, entonces $D \cap E=\emptyset$.

d) Si $\alpha_{1}$ y $\alpha_{2}$ son límites entonces $D \cap E$ es finito.

Por tanto, $\mathcal{D}$ es una familia casi ajena. Ahora, sea $E$ un subconjunto numerable y $\alpha=\sup \{\beta: E \backslash \beta$ es infinito $\}$ el supremo esencial de $E$. Entonces,

i) Si $\alpha$ es punto límite en $L$, entonces existe una sucesión en $E$ cuyo supremo es $\alpha$ y, en consecuencia, existe $D \in \mathcal{D}_{\alpha}$ tal que $E \cap D$ es infinito.

ii) Si $\alpha$ no es punto límite en $L$, entonces para algún $\beta<\mathfrak{c}^{+}$tal que $\alpha=\omega+\beta, E \cap[\beta, \alpha)$ es infinito, por definición de supremo esencial.

Así, $\mathcal{D}$ es una familia maximal casi ajena de $\mathfrak{c}^{+}$.

Aplicando el Lema 2.34, fijamos la familia $\mathcal{A}(D)$, los puntos $\left\{p_{A}: A \in\right.$ $\mathcal{A}(D)\}$ y el espacio $P(D)$ para cualquier $D \in \mathcal{D}$. Elegimos un nuevo punto $p$ y definimos $X=\{p\} \cup\left\{p_{A}: A \in \mathcal{A}(D), D \in \mathcal{D}\right\} \cup\left(\mathfrak{c}^{+} \times K\right)$ como el conjunto fundamental del espacio que queremos construir. Dado un punto $x=(\alpha, z) \in\left(\mathfrak{c}^{+} \times K\right)$, tomamos una base local de abiertos y cerrados $\left\{W_{n}: n \in \omega\right\}$ de $z$ en el espacio $K$ y sea $U_{n}=\{\alpha\} \times W_{n}$ para cada $n \in \omega$. Declaramos a la familia $\mathcal{B}_{x}=\left\{U_{n}: n \in \omega\right\}$ una base local del punto $x$ en $X$. Obsérvese que las bases locales así definidas generan la topología producto en $\mathfrak{c}^{+} \times K$, donde $\mathfrak{c}^{+}$es considerado con la topología discreta.

Si $A \in \mathcal{A}(D)$ para alguna $D \in \mathcal{D}$, entonces la base local $\mathcal{B}_{x}$ del punto $x=p_{A}$ es la misma que en el espacio $P(D)$. Finalmente, declaramos la base local de $X$ en el punto $p$ como $\mathcal{B}_{p}=\left\{V_{\alpha}: \alpha \in L\right\}$ donde $V_{\alpha}=\{p\} \cup(\{\beta$ : $\left.\left.\alpha<\beta<\mathfrak{c}^{+}\right\} \times K\right) \cup\left\{p_{A}: A \in \mathcal{A}(D), D \in \bigcup_{\beta>\alpha} \mathcal{D}_{\beta}\right\}$ para cada $\alpha \in L$. Ahora probaremos que $X$ tiene las propiedades requeridas. En efecto, $X$ es un espacio $T_{1}$ debido a que $X \backslash\{p\}$ es de Hausdorff y $\{p\}=\bigcap V_{p}$ donde 
$V_{p}$ es vecindad de $p$. Así, para probar que $X$ es de Tychonoff es suficiente mostrar que es cero-dimensional, para ello veremos que todos los miembros de las bases locales definidas anteriormente son abiertos y cerrados.

Si $x=(\alpha, z) \in \mathfrak{c}^{+} \times K$ y $U \in \mathcal{B}_{x}$, entonces $W=\left(\mathfrak{c}^{+} \times K\right) \backslash U$ es abierto en $\mathfrak{c}^{+} \times K$, por definición de $U$. Por tanto, $W$ es una vecindad de cualquier $y \in\left(\mathfrak{c}^{+} \times K\right) \backslash U$. Dado un $A \in \mathcal{A}(D)$ para algún $D \in \mathcal{D}$, se sigue de la definición de base local para $y=p_{A}$ que $\left\{p_{A}\right\} \cup\left[\left(\mathfrak{c}^{+} \times K\right) \backslash U\right]$ es una vecindad abierta de $y$ que no intersecta a $U$. Por otro lado, si $y=p$ entonces $V_{\beta} \cap U=\emptyset$ para cualquier $\beta \in L$ con $\beta>\alpha$. Por tanto, $U$ es un conjunto abierto y cerrado en $X$.

Ahora mostremos que si $x=p_{A}$ para alguna $A \in \mathcal{A}(D)$ con $D \in \mathcal{D}$ entonces cualquier $U \in \mathcal{B}_{x}$ es un conjunto abierto y cerrado. Para empezar, notemos que $W=\left(\mathfrak{c}^{+} \times K\right) \backslash U$ es un conjunto abierto y cerrado en $\mathfrak{c}^{+} \times K$, así que ningún punto de $W$ está en la cerradura de $U$. Si $y=p_{B}$ es distinto de $x$, entonces $B \in \mathcal{A}(D)$ o bien $\pi_{\mathfrak{c}^{+}}(A) \cap \pi_{\mathfrak{c}^{+}}(B)$ es finito porque la familia $\mathcal{D}$ es casi ajena. Obsérvese que $p_{B}$ pertenece a la cerradura de un conjunto $T \subset \mathfrak{c}^{+} \times K$ si y sólo si $T$ intersecta a una infinidad de conjuntos de la familia $\left\{B(d): d \in \pi_{\mathfrak{c}^{+}}(B)\right\}$. Si $B \in \mathcal{A}(D)$ no puede suceder que $A=T$, debido a que la familia $\mathcal{A}(D)$ es esencialmente disjunta. No obstante, si $B \in \mathcal{A}\left(D^{\prime}\right)$ para algún $D^{\prime} \neq D$, entonces $\pi_{\mathfrak{c}^{+}}(B) \cap \pi_{\mathfrak{c}^{+}}(A) \neq \emptyset$ es finito pues está contenido en el conjunto finito $D \cap D^{\prime}$. Finalmente, como $V_{\alpha} \cap U=\emptyset$ para cualquier $\alpha \in L$ con $\alpha>\sup (D)$, se sigue que $p$ no está en la cerradura de $U$.

Veamos que $V_{\alpha}$ es abierto y cerrado para cualquier $\alpha \in L$. Notemos que por definición $V_{\alpha} \cap\left(\mathfrak{c}^{+} \times K\right)$ es abierto y cerrado en $\mathfrak{c}^{+} \times K$, de modo que ningún punto de $\left(\mathfrak{c}^{+} \times K\right) \backslash V_{\alpha}$ puede estar en la cerradura de $V_{\alpha}$. Si $p_{A} \notin V_{\alpha}$, entonces $A \in \mathcal{A}(D)$ para algún $D \in \mathcal{D}_{\beta}$ con $\beta \leq \alpha$. Por definición de $\mathcal{D}_{\beta}$ tenemos que $\sup (D) \leq \alpha$ y, en consecuencia, $\left\{p_{A}\right\} \cup(D \times K)$ es una vecindaad de $p_{A}$ que no intersecta a $V_{\alpha}$. Así tenemos finalmente que $X$ es un espacio cero dimensional. Por tanto, $X$ es de Tychonoff.

Corroboremos que $X$ tiene estrechez no numerable. Como $\mathfrak{c}^{+} \times K$ tiene la topología discreta, $\mathfrak{c}^{+} \times K$ es denso en $X$. Si $\mathcal{U}=\left\{\{\alpha\} \times K: \alpha<\mathfrak{c}^{+}\right\}$ entonces $\bigcup \mathcal{U}$ es densa en $X$, de manera que $p \in c l_{X}(\bigcup \mathcal{U})$. No obstante, como cualquier vecindad $V_{\beta}$ de $p$ con $\beta>\mathfrak{c}$ satisface que $V_{\beta} \cap\left(\bigcup \mathcal{U}^{\prime}\right)=\emptyset$ donde $\mathcal{U}^{\prime}=\{\{\alpha\} \times K: \alpha<\mathfrak{c}\}$, resulta que $p \notin c l_{X}\left(\bigcup_{\mathcal{U}}^{\prime}\right)$ para cualquier $\mathcal{U}^{\prime} \subset \mathcal{U}$ con $\left|\mathcal{U}^{\prime}\right| \leq \mathfrak{c}$; en particular, $p$ no está en la cerradura de ningún subconjunto numerable de $\mathfrak{c}^{+} \times K$. Esto muestra que $X$ tiene estrechez no numerable. 
Para probar que $X$ es pseudocompacto tomamos una familia localmente finita $\mathcal{U}=\left\{U_{n}: n \in \omega\right\}$ de subconjuntos abiertos no vacíos de $X$. Como $\mathfrak{c}^{+} \times K$ es abierto y denso en $X$ podemos suponer que $U_{n}$ es un subconjunto abierto y cerrado para cada $n \in \omega$. Además, como cada $K_{\alpha}=\{\alpha\} \times K$ es compacto, sólo un número finito de $U_{n}$ 's intersectan a $K_{\alpha}$. Si $\pi_{\mathfrak{c}^{+}}(\bigcup \mathcal{U})$ es finito, entonces $\pi_{\mathfrak{c}^{+}}(\bigcup \mathcal{U}) \subseteq F$ para un subconjunto finito $F$ de $\mathfrak{c}^{+}$. Luego, $F \times K$ es un compacto que contiene a cada $U_{n}$. De modo que $\mathcal{U}$ tiene un punto de acumulación. Si $\pi_{\mathfrak{c}^{+}}(\bigcup \mathcal{U})$ es infinito se puede proceder del mismo modo que en la prueba del Lema 2.34 para obtener un conjunto admisible $A$. Por la maximalidad de $\mathcal{D}$ existe un conjunto $D \in \mathcal{D}$ tal que el conjunto $G=D \cap \pi_{\mathfrak{c}^{+}}(A)$ es infinito, pues $\pi_{\mathfrak{c}^{+}}(A)$ es infinito, pues $\pi_{\mathfrak{c}^{+}}(A)$ es infinito. Sea $A^{*}=\left\{a \in A: \pi_{\mathfrak{c}^{+}}(a) \in G\right\}$. De la construcción se sigue que $A^{*}$ es admisible y que $\pi_{\mathfrak{c}^{+}}\left(A^{*}\right) \subset G$. Por la maximalidad de la familia $\mathcal{A}(D)$ existe $B \in \mathcal{A}(D)$ tal que $B(\gamma) \cap A^{*}(\gamma) \neq \emptyset$ para una infinidad de $\gamma^{\prime}$ s. Luego, $p_{B}$ es un punto de acumulación de $A$ y de la familia $\left\{U_{n}: n \in \omega\right\}$, lo que es una contradicción. Por tanto, $X$ es pseudocompacto.

Ahora, veamos que $X$ es de Whyburn. Todos los puntos de $X \backslash\{p\}$ son puntos de Fréchet-Urysohn, por tratarse de puntos de primera numerabilidad. Por la Proposición 2.4, son de Whyburn. Así que, basta mostrar la propiedad para el punto p. Sea $T=\left\{p_{A}: A \in \mathcal{A}(D), D \in \mathcal{D}\right\}$. Supongamos que $p \in c l_{X}(A) \backslash A$ para algún $A \subset X$. Si $p \in c l_{X}(A \cap T)$, entonces $B=A \cap T$ es un conjunto casi cerrado en $A$, pues el único punto de acumulación de $B$ es $p$. Si no, entonces $p \in c l_{X}\left(A \cap\left(\mathfrak{c}^{+} \times K\right)\right)$ y, por tanto, $A^{\prime}=A \cap\left(\mathfrak{c}^{+} \times K\right)$ tiene cardinalidad $\mathfrak{c}^{+}$. Como sólo hay $\mathfrak{c}$ puntos en $K$, existe $t \in K$ tal que $B=\left\{\alpha<\mathfrak{c}^{+}:(\alpha, t) \in A^{\prime}\right\}$ tiene cardinalidad $\mathfrak{c}^{+}$. Si $F=B \times\{t\}$ entonces $F$ converge a $p$, por definición de las vecindades de $p$. Además, como todas las vecindades de los elementos de $T$ son admisibles, se sigue que a lo más pueden contener a un elemento de $F$. De manera que ningún elemento de $T$ pertenece a $c l_{X}(F)$, es decir, $T \cap c l_{X}(F)=\emptyset$. En consecuencia, $c l_{X}(F) \backslash F=\{p\}$, lo que nos permite concluir la demostración del teorema.

\section{3. $\quad P$-espacios}

En esta sección se estudia la propiedad de Whyburn en P-espacios.

2.36 Definición. $X$ es un P-espacio si cualquier subconjunto $G_{\delta}$ de $X$ es abierto, o equivalentemente, cada $F_{\sigma}$ es cerrado. En consecuencia, en un 
P-espacio y $T_{1}$, cada conjunto numerable es cerrado y discreto.

2.37 Teorema ([18]). Supongamos que $X$ es un P-espacio de Hausdorff, Lindelöf $y$ Whyburn. Entonces $t(X) \leq \omega_{1}$.

Demostración. Tomemos cualquier $A \subset X$ y cualquier $x \in c_{X}(A) \backslash A$. Como $X$ es Whyburn, existe $F \subset A$ tal que $c l_{X}(F) \backslash A=\{x\}$. Por el Lema 1.24, podemos tomar una familia maximal celular $\gamma$ de $F$ tal que $x \notin c l_{F}(V)$ para cualquier $V \in \gamma$. Observemos que $c l_{X}(F)$ es un $P$-espacio; en efecto, si $B$ es cualquier subconjunto $F_{\sigma}$ de $c l_{X}(F)$, entonces $B$ también es un subconjunto $F_{\sigma}$ en $X$ y, al estar en un $P$-espacio, se obtiene que $B$ es cerrado en $X$. Luego, la familia $\gamma$ es no numerable, porque de lo contrario, $\bigcup\left\{c l_{X}(V): V \in\right.$ $\gamma\}$ es un conjunto cerrado, por ser un conjunto $F_{\sigma}$ en un $P$-espacio, cuyo complemento en $F$ es $\{x\}$. Esto implica que $\{x\}$ es un punto aislado. Como $\gamma$ es maximal, $\bigcup \gamma$ es densa en $F$ y puesto que $x \in c l_{X}(F)$, se tiene que $x \in c l_{X}(\bigcup \gamma)$. Como $F$ es Whyburn, por la Proposición 2.5, existe $G \subset \bigcup \gamma$ tal que $c l_{X}(G) \backslash(\bigcup \gamma)=\{x\}$, donde $G$ es no numerable.

Para cualquier $V \in \gamma$ tal que $V \cap G \neq \emptyset$, elegimos un punto $x_{\mathrm{v}} \in V \cap G$. El conjunto $Q=\left\{x_{\mathrm{v}}: V \in \gamma\right.$ y $\left.V \cap G \neq \emptyset\right\}$ es discreto pues $Q \cap V=\left\{x_{\mathrm{v}}\right\}$. Además, $Q$ debe ser no numerable pues si $G$ sólo intersecta a un número numerable de elementos de $\gamma$, digamos $\left\{V_{n}: n \in \omega\right\}$, entonces $x \notin c l_{F}\left(V_{n}\right)$ para cada $n \in \omega$ y, como $X$ es un $P$-espacio, $\bigcup_{n \in \omega} c l_{X}\left(V_{n}\right)$ es cerrado en $X$. De modo que $x \notin c l_{X}\left(\bigcup_{n \in \omega} V_{n}\right)$ y, como $G \subset \bigcup_{n \in \omega} V_{n}$, resulta que $x \notin$ $c l_{X}(G)$. Esto es una contradicción, así que, $Q$ es no numerable. De hecho, cualquier subconjunto $E \subset Q$ es cerrado en $G$, ya que si $z \in c l_{G}(E)$ entonces $z \in \mathrm{cl}_{G}(Q)=c l_{X}(Q) \cap G \subset G$ y como $z \in G \subset \bigcup \gamma$, resulta que $z \in Q$. De manera que, $c l_{G}(E) \subset c l_{Q}(E)=E$, la igualdad se debe a que $Q$ es discreto. Entonces, $Q \backslash U$ es cerrado y discreto en $X$ para cualquier vecindad abierta $U$ de $x$. Como la propiedad de Lindelöf es hereditaria para cerrados, se tiene que $Q \backslash U$ es de Lindelöf y es numerable, por ser discreto. En consecuencia, si escogemos $\omega_{1}$ puntos de $Q$ se ha construido un conjunto $B \subset A$ tal que $|B| \leq \omega_{1}$ y $x \in \operatorname{cl}_{X}(B)$.

Un espacio $X$ se dice linealmente Lindelöf si, cualquier cubierta abierta ascendente tiene una subcubierta numerable o equivalentemente, si cualquier subconjunto no numerable $A$ de $X$ de cardinalidad regular tiene un punto de acumulación completa en $X$. Cualquier espacio linealmente Lindelöf de cardinalidad $\omega_{2}$ es de Lindelöf. 
2.38 Ejemplo ([18]). Existe un P-espacio, débilmente Whyburn, regular y Lindelöf de estrechez $\omega_{2}$. Así, no cualquier P-espacio, regular y Lindelöf es Whyburn.

Demostración. Consideremos $\omega_{2}+1$ con la topología del orden. La $\omega$-modificación $X$ de $\omega_{2}$ consiste en aislar cada ordinal de cofinalidad numerable. Veamos que $X$ es un $P$-espacio de Lindelöf. Basta corroborar que $X$ es linealmente Lindelöf. Sea $A \subset X$. Consideremos dos posibilidades:

1) Si $|A|=\omega_{1}$, tomemos $\alpha=\operatorname{mín}\left\{\beta \in \omega_{2}:|A \cap \beta|=\omega_{1}\right\}$. Veamos que $\alpha$ es punto de acumulación completa de $A$. Supongamos que no, entonces existe $\gamma \in \alpha$ tal que $|(\gamma, \alpha] \cap A|=\omega$, lo que implica que $|A \cap \gamma|=\omega_{1}$, contradiciedo la definición de $\alpha$.

2) Si $|A|=\omega_{2}$ entonces $\omega_{2}$ es punto de acumulación completa de $A$.

Como la topología de $X$ es más fuerte que la topología del orden de $\omega_{2}+1$, el espacio $X$ es disperso y, por el Teorema 2.27, es débilmente Whyburn. Sin embargo, $\omega_{2}$ está en la cerradura del conjunto de puntos aislados $E$ de $X$ y ningún subconjunto de $E$ de cardinalidad menor que $\omega_{2}$ tiene a $\omega_{2}$ en su cerradura. Esto muestra que $X$ no es de Whyburn, ya que cualquier $P$ espacio, Lindelöf y Whyburn tiene estrechez $\leq \omega_{1}$, por el Teorema 2.37 .

2.39 Proposición ([18]). Si $X$ es un P-espacio de Hausdorff con $\chi(X) \leq \omega_{1}$ entonces $X$ es Whyburn.

Demostración. Tomemos cualquier $A \subset X$ y cualquier $x \in \operatorname{cl}_{X}(A) \backslash A$. Sea $\left\{V_{\alpha}: \alpha<\omega_{1}\right\}$ una base local arbitraria de $x$ en $X$. Si $\beta<\omega_{1}$, entonces $U_{\beta}=\bigcap_{\alpha<\beta} V_{\alpha}$ es un conjunto abierto que contiene a $x$, por ser $X$ un $P$ espacio. Luego, $\mathcal{B}=\left\{U_{\beta}: \beta<\omega_{1}\right\}$ es una base local de $x$ tal que $U_{\beta} \subset U_{\gamma}$ si $\beta>\gamma$. Nótese que por tratarse de un $P$-espacio, cualquier subconjunto numerable de $X$ es cerrado. Elijamos un punto $x_{\alpha} \in U_{\alpha} \cap A$ para cada $\alpha<\omega_{1}$. Entonces el conjunto $F=\left\{x_{\alpha}: \alpha<\omega_{1}\right\} \subset A$ es casi cerrado y, como $\mathcal{B}$ es una base local en $x$, se tiene que $c l_{X}(F) \backslash A=\{x\}$. Por tanto, $X$ es Whyburn. 
2.40 Corolario ([18]). Si $X$ es un P-espacio de Hausdorff mismo que es Lindelöf con $\psi(X) \leq \omega_{1}$, entonces es Whyburn. En particular, si $X$ es un P-espacio, Lindelöf $y|X| \leq \omega_{1}$, entonces $X$ es un espacio Whyburn.

Demostración. Sean $C$ un cerrado no vacío de $X$ y $x \notin C$. Para todo $y \in C$, como los espacios que consideramos son Hausdorff, existen $U_{x}$ y $V_{y}$ abiertos ajenos de $x$ y $y$, respectivamente. Como $C \subset X$ es Lindelöf, existen $y_{n} \in C$ con $n \in \omega$ tales que $C \subset \bigcup_{n \in \omega} V_{y_{n}}=F$. Considerando que $X$ es un $P$ espacio, se tiene que $W=\bigcap_{n \in \omega} U_{x_{n}}$ es un abierto en $X$ que contiene a $x$ tal que $F \cap W=\emptyset$. Así, $X$ es regular.

Sean $x \in X$ y $\mathcal{B}=\left\{U_{\alpha}: \alpha<\omega_{1}\right\}$ una pseudobase local de $x$. Veamos que $\mathcal{C}=\left\{\bigcap_{\alpha<\beta} U_{\alpha}: \beta<\omega_{1}\right\}$ es una base local en $x$. Por la regularidad de $X$ podemos suponer que $c l_{X}\left(U_{\alpha}\right) \subseteq U_{\beta}$ si $\alpha>\beta$. Además, como $\mathcal{B}$ es pseudobase, $\bigcap_{\alpha<\omega_{1}} U_{\alpha}=\{x\}$. Sea $V$ una vecindad abierta de $x$. Entonces $X \backslash V$ es cerrado en $X$, lo que implica que es Lindelöf. Así, $\left\{X \backslash c l_{X}\left(U_{\alpha}\right)\right.$ : $\left.\alpha<\omega_{1}\right\}$ es una cubierta abierta de $X \backslash V$. Como $X \backslash V$ es Lindelöf, existe $\beta<\omega_{1}$ tal que $\left\{X \backslash c l_{X}\left(U_{\alpha}\right): \alpha<\beta\right\}$ es una subcubierta abierta numerable de $X \backslash V$. Luego, como $\bigcap_{\alpha<\beta} c l_{X}\left(U_{\alpha}\right) \subseteq V$, resulta que $\mathcal{C}$ es una base local de $x$. Por tanto, $\chi(X) \leq \omega_{1}$. Finalmente, por la Proposición $2.39, X$ es de Whyburn.

Para finalizar la sección, bajo un axioma muy fuerte, se contesta negativamente a la pregunta hecha en [18] de si zes cierto que cualquier P-espacio es débilmente Whyburn? Para tal efecto, haremos referencia al prototipo más conocido de grandes cardinales, los cardinales medibles; cuyo origen proviene de los trabajos de Banach, Kuratowski, Tarski y Ulam alrededor de 1930.

2.41 Definición. Un ultrafiltro $\mathcal{U}$ en un conjunto $X$ es uniforme si todos los elementos en $\mathcal{U}$ tienen la misma cardinalidad que $X$.

2.42 Definición. Un filtro $\mathcal{F}$ es $\kappa$-completo siempre que $\mathcal{G} \subseteq \mathcal{F}$ y $|\mathcal{G}|<\kappa$, entonces $\bigcap \mathcal{G} \neq \emptyset$. Todo filtro es $\omega$-completo.

La pregunta sobre la existencia de ultrafiltros $\omega_{1}$-completos no principales en conjuntos no numerables es un problema muy famoso en teoría de conjuntos, aunque se sabe que consistentemente no existen. A continuación introduciremos el concepto de cardinal medible en términos de ultrafiltros. 
2.43 Definición. El cardinal más pequeño a para el que existe un ultrafiltro $\omega_{1}$-completo es llamado el primer cardinal medible.

2.44 Observación. Ningún conjunto numerable puede pertenecer a un ultrafiltro $\omega_{1}$-completo.

2.45 Teorema. Si existe un cardinal medible, existe un P-espacio de Tychonoff que no es débilmente Whyburn.

Demostración. Sean $\kappa$ el primer cardinal medible y $D_{\kappa}$ el espacio discreto de cardinalidad $\kappa$. Denotamos por $X$ a la $\omega$-modificación de $\beta D_{\kappa}$. Luego, $X$ es un $P$-espacio y se sabe que $X$ es un espacio de Tychonoff.

Afirmamos que $X$ no es un espacio débilmente Whyburn. Nótese que existe un ultrafiltro $\omega_{1}$-completo $\mathcal{F}$ en $D_{\kappa}$; de ahí que, si $S \subseteq D_{\kappa}$ tiene cardinalidad $\kappa$, entonces hay una biyección $f: D_{\kappa} \longrightarrow S$ y $f[\mathcal{F}]$ es un ultrafiltro $\omega_{1}$-completo en $S$, de donde, $S$ es un elemento de un ultrafiltro $\omega_{1}$ completo en $D_{\kappa}$. Como cada conjunto $A$ de cardinalidad $\kappa$, puede ser dividido en un número infinito de subconjuntos de cardinalidad $\kappa$, se sigue que cada subconjunto $A \subseteq D_{\kappa}$ de cardinalidad $\kappa$ es un elemento de un número infinito de ultrafiltros $\omega_{1}$-completos en $D_{\kappa}$.

Si $A \subseteq D_{\kappa}$ y $A$ es de cardinalidad $\kappa$, entonces $c l_{\beta D_{\kappa}}(A)$ contiene un número infinito de ultrafiltros $\omega_{1}$-completos. Además, si $\mathcal{U} \in c l_{\beta D_{\kappa}}(A)$ es $\omega_{1}$-completo, se sigue que cada $G_{\delta}$ en $\beta D_{\kappa}$ que contiene a $\mathcal{U}$ intersecta a $A$; de donde, $\mathcal{U} \in c l_{X}(A)$. Por otro lado, si $B \subseteq D_{\kappa}$ tiene cardinalidad no medible, entonces cualquier elemento de $c l_{D_{\kappa}}(B)$ es un ultrafiltro que no es $\omega_{1}$-completo. Por tanto, para cada $\mathcal{V} \in c_{D_{\kappa}}(B)$, existe un conjunto numerable $\left\{V_{n}: n \in \omega\right\} \subseteq \mathcal{V}$ tal que $\bigcap\left\{V_{n}: n \in \omega\right\}=\emptyset$. Así que $\mathcal{V} \notin c l_{X}(B)$.

Se ha mostrado que $D_{\kappa}$ no es cerrado en $X$, pero que cada subconjunto de $D_{\kappa}$ con cardinalidad no medible es cerrado en $X$ y cada conjunto $A \subseteq D_{\kappa}$ que tiene cardinalidad medible es tal que $c l_{X}(A) \backslash A$ es infinita. Por tanto, $X$ no es débilemente Whyburn.

\subsection{Una Caracterización en Espacios Lineal- mente Ordenados}

Sea $L$ un espacio linealmente ordenado. Denotamos por $L^{*}$ a la completación de Dedekind del espacio $L$ y por $<$ al orden de ambos. Para evi- 
tar posibles confusiones denotaremos a los intervalos haciendo énfasis en el conjunto a considerar. Así, dado un punto $x \in L$, tenemos los intervalos $(\leftarrow, x]_{L}=\{y \in L: y \leq x\}, \quad(\leftarrow, x]_{L^{*}}=\left\{y \in L^{*}: y \leq x\right\}, \quad[x, \rightarrow)_{L}=\{y \in$ $L: x \leq y\}$ y $[x, \rightarrow)_{L^{*}}=\left\{y \in L^{*}: x \leq y\right\}$, los intervalos faltantes se definen similarmente.

2.46 Definición. A un espacio linealmente ordenado L lo llamaremos incompleto izquierdo en el punto $x \in L$, si existe un conjunto $L^{*}$-casi cerrado $F \subset\left(L^{*} \backslash L\right) \cap(\leftarrow, x)_{L^{*}}$ tal que $F \rightarrow x$.

Análogamente, el espacio $L$ es incompleto derecho en el punto $x \in L$, si existe un conjunto $L^{*}$-casi cerrado $F \subset\left(L^{*} \backslash L\right) \cap(x, \rightarrow)_{L^{*}}$ tal que $F \rightarrow x$.

2.47 Definición. Un espacio linealmente ordenado L es un club incompleto si, para cualquier $x \in L$ con $\chi\left(x,(\leftarrow, x]_{L}\right)>\omega$ (respectivamente, $\left.\chi\left(x,[x, \rightarrow)_{L}\right)>\omega\right)$, el espacio $L$ es incompleto izquierdo (derecho) en el punto $x$.

2.48 Teorema ([18]). Un espacio topológico linealmente ordenado L es Whyburn si y sólo si es club incompleto.

Demostración. Supongamos que $L$ es club incompleto, $A \subset L$ y $x \in c l_{L}(A) \backslash$ $A$. Sin pérdida de generalidad, podemos suponer que $A \subset(\leftarrow, x)_{L}$ o que $A \subset(x, \rightarrow)_{L}$. Consideremos $A \subset(\leftarrow, x)_{L}$. Si $\left.\chi(x,(\leftarrow, x])_{L}\right)=\omega$, entonces no hay nada que probar. Por eso asumimos que $\left.\chi(x,(\leftarrow, x])_{L}\right)=\kappa>\omega$ y fijemos un conjunto $L^{*}$-casi cerrado $F \subset\left(L^{*} \backslash L\right) \cap(\leftarrow, x)_{L^{*}}$ tal que $F \rightarrow x$. Como en los espacios linealmente ordenados el carácter de cada punto es regular, construimos puntos $\left\{x_{\alpha}, y_{\alpha}, z_{\alpha}: \alpha<\kappa\right\}$ con las siguientes propiedades:

(1) $x_{\alpha} \in A$ y $y_{\alpha}, z_{\alpha} \in F$ para cada $\alpha<\kappa$;

(2) $y_{\alpha}<x_{\alpha}<z_{\alpha}$ para cada $\alpha<\kappa$ :

(3) Si $\alpha<\beta<\kappa$ entonces $z_{\alpha}<y_{\beta}$.

Como el conjunto $B=\left\{x_{\alpha}: \alpha<\kappa\right\}$ es cofinal en $(\leftarrow, x)_{L}$, se tiene que $B \rightarrow x$ y que $x \in c l_{L}(B)$. Probaremos que $c l_{L}(B) \backslash B=\{x\}$. Sea $a<x$ cualquier punto de acumulación de $B$ en $L^{*}$, entonces $a=\sup \left\{x_{\alpha}: \alpha<\beta\right\}$ para algún $\beta<\kappa$. Luego, (2) y (3) implican que para los conjuntos $C=$ $\left\{y_{\alpha}: \alpha<\beta\right\}$ y $D=\left\{z_{\alpha}: \alpha<\beta\right\}$ tenemos que $\sup (C)=\sup (D)=a$. Considerando que el conjunto $F \cap(\leftarrow, a]_{L^{*}}$ es cerrado, se tiene que $a \in F \subset$ 
$L^{*} \backslash L$, esto es, $a \notin L$. En consecuencia, ningún punto de $L \backslash(B \cup\{x\})$ está en $c l_{L}(B)$. De manera que el conjunto $B$ es casi cerrado. Por tanto, $L$ es un espacio Whyburn.

Sea $L$ un espacio Whyburn. Supongamos que $\chi\left(x,(\leftarrow, x]_{L}\right)>\omega$. Entonces $x \in c l_{L}\left((\leftarrow, x)_{L}\right)$ y existe un espacio discreto $D \subset(\leftarrow, x)_{L}$ con $x \in c l_{L}(D)$, por la Proposición 1.26. Como $L$ es de Whyburn, existe un conjunto casi cerrado $B \subset D$ con $B \rightarrow x$. El conjunto $F=\left(c l_{L^{*}}(B) \backslash B\right) \cap(\leftarrow, x)_{L^{*}}$ es un subconjunto $L^{*}$-casi cerrado de $\left(L^{*} \backslash L\right) \cap(\leftarrow, x)_{L^{*}}$ tal que $F \rightarrow x$.

2.49 Definición. Un espacio $X$ es Lindelöf $\Sigma$ si $X$ es una imagen continua de un espacio $Y$ que puede ser perfectamente mapeado sobre una espacio segundo numerable.

2.50 Lema. La clase de los espacios Lindelöf $\Sigma$ es invariante bajo subespacios cerrados.

Demostración. Supongamos que $X$ es un espacio Lindelöf $\Sigma$. Sea $f: Y \longrightarrow$ $X$ una función continua suprayectiva y sea $g: Y \longrightarrow Z$ una función perfecta, donde $Z$ es segundo numerable. Sea $A \subset X$ un subconjunto cerrado. Entonces $F=f^{-1}(A)$ es un subconjunto cerrado de $Y$.

La función $\left.g\right|_{F}: F \longrightarrow g(F)$ es perfecta. En efecto, sea $B$ cualquier subconjunto cerrado de $F$. Como $F$ es cerrado en $Y$, se tiene que $B$ es cerrado en $Y$. Dado que la función $g$ es cerrada, $g(B)$ es cerrado en $Z$ y, como $g(B) \subset g(F)$, se tiene que $g(B)$ es cerrado en $g(F)$, lo que significa que $\left.g\right|_{F}$ es cerrada. Por otro lado, como $g$ es perfecta, dada una cubierta abierta $\left\{U_{\alpha}: \alpha \in \Lambda\right\}$ de $g^{-1}(z)$ para cualquier $z \in Z$, existen $\alpha_{1}, \alpha_{2}, \ldots, \alpha_{n} \in \Lambda$ tales que $g^{-1}(z) \subset \bigcup_{i=1}^{n} U_{\alpha_{i}}$. Así, $\left(\left.g\right|_{F}\right)^{-1}(z) \subset \bigcup_{i=1}^{n}\left(U_{\alpha_{i}} \cap F\right)$ por lo que $\left(\left.g\right|_{F}\right)^{-1}(z)$ es compacto para cada $z \in g(F)$. Por tanto, $\left.g\right|_{F}$ es perfecta. Además, como cualquier subconjunto de un espacio segundo numerable es segundo numerable, resulta que $A$ es Lindelöf $\Sigma$. Es decir, la propiedad es invariante bajo subespacios cerrados.

2.51 Proposición. Sea $L$ un espacio de Tychonoff, linealmente ordenado y Lindelöf $\Sigma$. Entonces $L$ es Whyburn si y sólo si es primero numerable.

Demostración. Como $t(L)=\chi(L)$ en espacios linealmente ordenados (ver [11], 3.12.4(d)), es suficiente probar que si $L$ tiene la propiedad de Whyburn 
entonces $t(L) \leq \omega$. Supongamos que $A \subset L$ y $x \in c_{L}(A) \backslash A$. Sin pérdida de generalidad supongamos que $A \subset(\leftarrow, x)$ o $A \subset(x, \rightarrow)$. Como los casos son idénticos asumimos que $A \subset(\leftarrow, x)$. Por la Proposición 1.26, existe un subespacio discreto $D \subset A$ con $x \in c l_{L}(D)$. Como $L$ es de Whyburn, existe un conjunto $B \subset D$ tal que $c l_{L}(B) \backslash D=\{x\}$. Si $U$ es cualquier vecindad abierta del punto $x$, el conjunto $B \backslash U$ es cerrado y discreto en $L$. Luego, como $B \backslash U$ es cerrado, hereda la propiedad de Lindelöf y como en un espacio de Lindelöf un discreto cerrado es a lo más numerable, $B \backslash U$ es numerable. Además, $B \cup\{x\}$ es un espacio Lindelöf $\Sigma$, por el Lema 2.50. Si $x \in c l_{L}(C)$ para algún $C \subset B$ numerable entonces habremos terminado. Si no, entonces $B \cup\{x\}$ es homeomorfo a la Lindelöficación en un punto de un espacio discreto no numerable $Y$. Nótese que todo subconjunto compacto $C \subset Y$ es finito, pues de lo contrario, si $C$ es infinito, cerrado y discreto, entonces $C$ no es numerablemente compacto y tampoco compacto. Entonces, por ([4], Proposición IV. 6.15), $B \cup\{x\}$ no es Lindelöf $\Sigma$. Esta contradicción muestra que $t(L) \leq \omega$. Por tanto, $L$ es primero numerable.

Inversamente, cualquier espacio primero numerable de Hausdorff es de Whyburn.

El siguiente ejemplo muestra que la Proposición 2.51 no es cierta si sólo se supone la propiedad de Lindelöf de $L$.

2.52 Ejemplo. Existe un espacio linealmente ordenado, Lindelöf y de Whyburn de estrechez no numerable.

Demostración. El ejemplo a considerar será la recta larga definida como $M=\left(\omega_{1} \times[0,1)\right) \cup\left\{\left(\omega_{1}, 0\right)\right\}$ con el orden lexicográfico. Notemos que el complemento de un abierto en $M$ que contiene a $\omega_{1}$ es compacto, de donde $M$ es compacto y, en consecuencia, completo (ver $[6])$. Notemos que $\left(\omega_{1}, 0\right)$ es el único punto con carácter no numerable. Si $L=M \backslash\left(\omega_{1} \times\{0\}\right)$, entonces $M=L^{*}$ y $F=\omega_{1} \times\{0\}$ es un subconjunto $L^{*}$-casi cerrado de $\left(L^{*} \backslash L\right) \cap\left(\leftarrow,\left(\omega_{1}, 0\right)\right)_{L^{*}}$ tal que $F \rightarrow\left(\omega_{1}, 0\right)$, es decir, $L$ es club incompleto. Aplicando el Teorema 2.48 concluimos que $L$ es un espacio de Whyburn. Luego, como $L \backslash U$ es segundo numerable para cada abierto $U$ que contiene a $\omega_{1}, L$ es Lindelöf. Finalmente, si $A=\omega_{1} \times\left\{\frac{1}{2}\right\}$, entonces $\left(\omega_{1}, 0\right) \in c l_{L}(A)$ pero ningún subconjunto numerable $B$ de $A$ es tal que $\left(\omega_{1}, 0\right) \in c l_{L}(B)$. Por tanto, la estrechez de $L$ es no numerable. 


\subsection{Propiedad de Whyburn en Productos}

En la presente sección los espacios a considerar son de Tychonoff. Se da una condición suficiente para que el producto de dos espacios tenga la propiedad débilmente Whyburn.

Las funciones $\pi_{X}: X \times Y \longrightarrow X$ y $\pi_{Y}: X \times Y \longrightarrow Y$ representan las proyecciones naturales.

2.53 Teorema ([8]). El producto de un espacio compacto semiradial con un espacio compacto débilmente Whyburn es un espacio débilmente Whyburn.

Demostración. Supongamos por contradicción que existe un espacio $X$ compacto semiradial y un espacio $Y$ compacto débilmente Whyburn tal que $X \times Y$ no es débilmente Whyburn. Entonces existe un subconjunto Whyburn cerrado $A \subset X \times Y$ que no es cerrado. Sea $\kappa$ el mínimo cardinal tal que el conjunto $A$ no es $\kappa$-cerrado y escogemos un conjunto $B \subset A$ de cardinalidad $\kappa$ tal que $c l_{X \times Y}(B) \backslash A \neq \emptyset$. Tomemos un punto $(x, y) \in c l_{X \times Y}(B) \backslash A$. Observemos que $\{x\} \times Y$ es débilmente Whyburn por ser homeomorfo a $Y$ y $A \cap(\{x\} \times Y)$ es Whyburn cerrado, debido a que $\{x\} \times Y$ es cerrado y $A$ es Whyburn cerrado en $X \times Y$. Por regularidad de $X \times Y$, existe una vecindad cerrada $V$ de $(x, y)$ tal que $V \cap[A \cap(\{x\} \times Y)]=\emptyset$. Cambiando $A$ por $A \cap V$, podemos suponer que $x \notin \pi_{X}(A)$. Recordando que $(x, y) \in c l_{X \times Y}(B) \backslash A$, se tiene que $x \in \pi_{X}\left(c l_{X \times Y}(B)\right) \backslash \pi_{X}(A)$ y, por continuidad, $x \in \pi_{X}\left(c l_{X \times Y}(B)\right) \subset c l_{X}\left(\pi_{X}(B)\right)$, de donde, $\pi_{X}(A)$ no es $\kappa$-cerrada.

Puesto que $X$ es semiradial podemos fijar $\lambda \leq \kappa$ y una cadena $\left\{x_{\xi}\right.$ : $\xi \in \lambda\} \subset \pi_{X}(A)$ que converge a un punto $\widehat{x} \in X \backslash \pi_{X}(A)$. Nótese que $A$ es $<\kappa$-cerrado, debido a que $\kappa$ es el mínimo cardinal tal que $A$ no es $\kappa$ cerrado y por tratarse de espacios compactos de Hausdorff, la proyección es un mapeo cerrado. Por la Proposición 1.15, $\pi_{X}(A)$ es $<\kappa$-cerrado, de manera que $\lambda \geq \kappa$. Luego, $\lambda=\kappa$ y $\kappa$ es cardinal regular, pues de lo contrario habría una subcadena de cofinalidad menor que $\kappa$ que sale de $\pi_{X}(A)$.

Para cualquier $\xi \in \kappa$, elegimos un $y_{\xi}$ tal que $\left(x_{\xi}, y_{\xi}\right) \in A$. Como $Y$ es compacto, podemos escoger un punto de acumulación completa $p \in Y$ del conjunto $\left\{y_{\xi}: \xi \in \kappa\right\}$. De manera que $(\widehat{x}, p) \in c l_{X \times Y}(A) \backslash A$. Notemos que $X \times\{p\}$ es débilmente Whyburn por ser homeomorfo a $X$, ya que por la Proposición 2.20, $X$ es débilmente Whyburn; además, como $X \times\{p\}$ es cerrado y $A$ es Whyburn cerrado, $A \cap(X \times\{p\})$ es Whyburn cerrado. Por regularidad de $X \times Y$, existe una vecindad cerrada $W$ de $(\widehat{x}, p)$ tal que $W \cap$ 
$[A \cap(X \times\{p\})]=\emptyset$. Reemplazando $A$ por $W \cap A$, podemos suponer que $p \notin \pi_{Y}(A)$.

Para cualquier $\xi \in \kappa$, sean $C_{\xi}=c l_{Y}\left(\left\{y_{\nu}: \nu \in \xi\right\}\right)$ y $C=\bigcup_{\xi \in \kappa} C_{\xi}$. Puesto que la proyección entre espacios compactos de Hausdorff es un mapeo cerrado, por la Proposición 1.15, $\pi_{Y}(A)$ es $<\kappa$-cerrado. Como $C_{\xi}$ es la cerradura de un conjunto de cardinalidad menor que $\kappa$, se sigue que $C \subset \pi_{Y}(A)$. Además, como $p$ es punto de acumulación completa, resulta que $p \in c_{Y}(C) \backslash \pi_{Y}(A)$, lo que quiere decir que $C$ no es cerrado en $Y$. Puesto que $Y$ es débilmente Whyburn, existen $D \subset C$ y un punto $\widehat{y} \notin C$ tales que $c l_{Y}(D) \backslash C=\{\widehat{y}\}$. De modo que podemos escribir $c l_{Y}(D)=\{\widehat{y}\} \cup\left(C \cap c l_{Y}(D)\right)=\{\widehat{y}\} \cup\left[\bigcup_{\xi \in \kappa}\left(c l_{Y}(D) \cap C_{\xi}\right)\right]$.

Por la regularidad de $c l_{Y}(D)$, para cada $\xi \in \kappa$ elegimos una vecindad cerrada $U_{\xi}$ de $\widehat{y}$ en $c l_{Y}(D)$ tal que $U_{\xi} \cap\left[c l_{Y}(D) \cap C_{\xi}\right]=\emptyset$. Sea $V_{\xi}=\bigcap_{\nu \in \xi} U_{\nu}$. Notemos que $c l_{Y}(D)$ es compacto y $c l_{Y}(D) \backslash\{\widehat{y}\}$ es $<\kappa$-cerrado. Supongamos que $V_{\xi} \backslash\{\widehat{y}\}=\emptyset$, entonces $V_{\xi}=\{\widehat{y}\}=\bigcap_{\nu \in \xi} U_{\nu}$ para algún $\xi<\kappa$. Digamos que $\xi$ es el mínimo con esta propiedad y escogemos un punto $u_{\nu} \in V_{\nu} \backslash\{\widehat{y}\}$ para cada $\nu<\xi$. Entonces $\left\{u_{\nu}: \nu \in \xi\right\}$ es una cadena cuyo único punto de acumulación es $\widehat{y}$, es decir, $c_{Y}(D) \backslash\{\widehat{y}\}$ no es $<\kappa$-cerrado, lo cual no puede suceder. Por tanto, $V_{\xi} \backslash\{\widehat{y}\} \neq \emptyset$ para cada $\xi<\kappa$.

Ahora, tomando un punto $z_{\xi} \in V_{\xi} \backslash\{\widehat{y}\}$ para cualquier $\xi \in \kappa$, obtenemos una cadena que converge a $\widehat{y}$. Como $U_{\xi} \cap C_{\xi}=\emptyset$ se sigue que $V_{\xi} \cap C_{\xi}=$ $\emptyset$. Puesto que $z_{\xi} \in V_{\xi} \backslash\{\widehat{y}\} \subseteq c l_{Y}(D) \backslash\{\widehat{y}\} \subseteq C$ se tiene que $z_{\xi} \in C_{\gamma}$ para alguna $\gamma<\kappa$. No obstante, como $z_{\xi} \in V_{\xi}$ y $V_{\xi} \cap C_{\xi}=\emptyset$, resulta que $z_{\xi} \notin c l_{Y}\left(\left\{y_{\nu}: \nu \in \xi\right\}\right)$, lo que implica que $z_{\xi} \in c l_{Y}\left(\left\{y_{\nu}: \xi \leq \nu<\gamma\right\}\right)$. Por eso hay una red $S=\left\{y_{\nu}\right\}_{\nu \in D} \subset\left\{y_{\nu}: \xi \leq \nu<\gamma\right\}$ que converge a $z_{\xi}$. Sea $S^{\prime}=\left\{\left(x_{\nu}, y_{\nu}\right)\right\}_{\nu \in D^{\prime}}$ la red correspondiente en $A$. Como $X \times Y$ es compacto, $S^{\prime}$ tiene una subred $S^{\prime \prime}=\left\{\left(x_{\nu}, y_{\nu}\right)\right\}_{\nu \in D^{\prime \prime}}$ que converge; además, dado que $\left|\left\{y_{\nu}\right\}_{\nu \in D^{\prime \prime}}\right|<\kappa$, su límite está en $A$. Sea $w_{\xi}$ el límite de la primera coordenada. Entonces $w_{\xi} \in c l_{X}\left(\left\{x_{\nu}: \xi \leq \nu<\gamma\right\}\right)$ para alguna $\gamma<\kappa$ y $\left(w_{\xi}, z_{\xi}\right) \in A$.

Recordando que $x_{\alpha}$ converge a $\widehat{x}$, dada cualquier vecindad cerrada $U$ de $\widehat{x}$ existe $\beta<\kappa$ tal que para cada $\alpha>\beta$ sucede que $x_{\alpha} \in U$. Si $\xi>\beta$, entonces $w_{\xi} \in\left\{x_{\nu}: \xi \leq \nu<\gamma\right\} \subseteq c_{X}\left(\left\{x_{\alpha}: \alpha>\beta\right\}\right) \subseteq c l_{X}(U)=U$, lo que muestra que $\left\{w_{\xi}: \xi<\kappa\right\}$ converge a $\widehat{x}$. Por tanto, $F=\left\{\left(w_{\xi}, z_{\xi}\right): \xi \in \kappa\right\}$ converge a $(\widehat{x}, \widehat{y}) \notin A$.

Cualquier punto $(a, b) \in c l_{X \times Y}(F)$ distinto de $(\widehat{x}, \widehat{y})$ está en la cerradura de un segmento inicial de $F$, ya que de lo contrario, cualquier vecindad de $(a, b)$ contiene a un segmento final de $F$, lo que no puede suceder porque el espacio es de Hausdorff. De manera que, cualquiera de estos puntos $(a, b)$ 
están en $A$, pues $A$ es $<\kappa$-cerrado. Por lo tanto, $c l_{X \times Y}(F) \backslash A=\{(\widehat{x}, \widehat{y})\}$, lo que contradice que $A$ sea Whyburn cerrado.

2.54 Corolario ([8]). El producto de un espacio compacto y débilmente Whyburn con el intervalo $[0,1]$ es un espacio débilmente Whyburn.

Demostración. Basta notar que el intervalo $[0,1]$ es compacto y primero numerable.

2.55 Corolario ([8]). El producto de un espacio compacto y débilmente Whyburn $X$ con un espacio compacto Whyburn $Y$ es un espacio débilmente Whyburn.

Demostración. Para aplicar el Teorema 2.53, basta notar que por la Proposición $2.22, Y$ es radial.

El siguiente teorema muestra esencialmente que el producto de un espacio Whyburn con un espacio compacto y primero numerable no tiene que ser un espacio débilmente Whyburn.

2.56 Teorema ([7]). El producto de un espacio numerable y submaximal con una sucesión convergente no es débilmente Whyburn.

Demostración. Sea $X$ el producto de un espacio numerable y submaximal $C$ con la sucesión convergente $\omega+1$. Fijemos una biyección arbitraria $f: C \rightarrow \omega$. El conjunto $A=\{(x, f(x)): x \in C\}$ no es cerrado en $X$. En efecto, sea $U=V \times W$ una vecindad de $(x, \omega)$, entonces $|V|=\omega$ y $\omega \backslash W$ es finito. Luego, existe $y \in V$ tal que $f(y) \in W$, de manera que $(y, f(y)) \in U \cap A$. Por tanto, $A$ no es cerrado. Supongamos que existe $B^{\prime} \subset C$, tal que para $B=\left\{(x, f(x)): x \in B^{\prime}\right\}$ se tenga que $\left|c l_{X}(B) \backslash A\right|=1, \mathrm{y}$, por lo tanto, $c l_{X}(B) \backslash A=\{(y, \omega)\}$ para algún $y \in C$. Notemos que $|B \cap(\{y\} \times \omega)| \leq 1$. Si $y \notin c l_{C}\left(B^{\prime} \backslash\{y\}\right)$, entonces existe una vecindad $U^{\prime}$ de $y$ tal que $U^{\prime} \cap\left(B^{\prime} \backslash\right.$ $\{y\})=\emptyset$. Entonces, existe una vecindad $V^{\prime}$ de $(y, \omega)$ tal que $V^{\prime} \cap B=\emptyset$, lo que no puede suceder. Por tanto $y \in \operatorname{cl}_{C}\left(B^{\prime} \backslash\{y\}\right)$ y, en consecuencia, $B^{\prime} \backslash\{y\}$ no es cerrado. Así, int $\operatorname{in}_{C}\left(B^{\prime} \backslash\{y\}\right) \neq \emptyset$. Tomando un punto $z \in \operatorname{int}_{C}\left(B^{\prime} \backslash\{y\}\right)$ se tiene que $(z, \omega) \in c l_{X}(B) \backslash A$, lo que contradice que $c l_{X}(B) \backslash A=\{(y, \omega)\}$. De aquí $\left|c l_{X}(B) \backslash A\right| \neq 1$. Luego, $A$ es Whyburn cerrado y, por definición, no es cerrado. Por tanto, $X$ no es débilmente Whyburn. 
Una consecuencia importante de la Proposición 2.32 y del Teorema 2.56 es el siguiente:

2.57 Teorema ([7]). Existe un espacio numerable y Whyburn $C$ tal que el producto de $C$ con una sucesión convergente no es débilmente Whyburn.

2.58 Corolario ([7]). Existe un espacio numerable que no es débilmente Whyburn.

2.59 Proposición ([7]). El producto de un espacio secuencial $X$ con un espacio numerablemente compacto y débilmente Whyburn $Y$ es débilmente Whyburn.

Demostración. Sean $A \subset X \times Y$ y $(x, y) \in\left[c l_{X \times Y}(A)\right] \backslash A$. Si $A \cap(\{x\} \times Y)$ no es cerrado, usando el hecho de que $\{x\} \times Y$ es débilmente Whyburn, por ser homeomorfo a $Y$, existe un subconjunto $B \subset A$ tal que $c l_{X \times Y}(B) \backslash A=$ $\{(x, z)\}$ para algún $z \in Y$.

Si $A \cap(\{x\} \times Y)$ es cerrado podemos suponer, por el proceso usado en el Teorema 2.53, que $A \cap(\{x\} \times Y)=\emptyset$. Como $(x, y) \in c l_{X \times Y}(A) \backslash A$ entonces $x \in \pi_{X}\left(c l_{X \times Y}(A)\right) \backslash \pi_{X}(A)$ y, por continuidad, $x \in c l_{X}\left(\pi_{X}(A)\right)$ lo que significa que $\pi_{X}(A)$ no es cerrada en $X$. Así, existe una sucesión $\left\{x_{n}\right.$ : $n \in \omega\} \subset \pi_{X}(A)$ que converge a $x^{\prime} \in X \backslash \pi_{X}(A)$. Para cada $n \in \omega$ tomemos un punto $y_{n} \in Y$ de manera que $\left(x_{n}, y_{n}\right) \in A$. Por la Proposición 2.23, $Y$ es secuencialmente compacto. Así, $\left\{y_{n}: n \in \omega\right\}$ contiene una subsucesión $\left\{y_{n_{k}}: k \in \omega\right\}$ que converge a un punto $y$. Luego, $F=\left\{\left(x_{n_{k}}, y_{n_{k}}\right): k \in \omega\right\}$ converge a $\left(x^{\prime}, y\right)$. Además, como se trata de espacios de Tychonoff, $F$ tiene un único punto límite. Así que, $c l_{X \times Y}(F)=F \cup\left\{\left(x^{\prime}, y\right)\right\}$. De modo que $c l_{X \times Y}(F) \backslash A=\left\{\left(x^{\prime}, y\right)\right\}$. Por tanto, $X \times Y$ es débilmente Whyburn.

Ahora mostraremos que la propiedad de Whyburn puede no satisfacerse aún en el producto de un espacio de Fréchet-Urysohn con un espacio métrico compacto.

2.60 Ejemplo ([7]). Existe un espacio Fréchet-Urysohn numerable y un espacio métrico compacto y numerable, cuyo producto no es Whyburn.

Demostración. Sea $S_{\omega}=\{0\} \cup\{(n, m): n, m \in \omega\}$ un abanico numerable de Fréchet-Urysohn, cuyo único punto no aislado es el cero. Representamos por 
$m$ a la sucesión que converge a cero y por $n$ a la "espina" correspondiente. Afirmamos que el producto $X=S_{\omega} \times(\omega+1)^{2}$ del espacio Fréchet-Urysohn $S_{\omega}$ con el espacio métrico compacto $(\omega+1)^{2}$ no es de Whyburn.

Sean $A=\{((n, m),(n, m)): n, m \in \omega\}$ la "diagonal" tomada sobre los puntos aislados de ambos factores de $X$ y $V=U \times([n, \omega] \times[n, \omega])$ una vecindad arbitraria de $(0,(\omega, \omega))$. Para cada espina $n$ existe $k \in \omega$ tal que para toda $m_{n} \geq k,\left(n, m_{n}\right) \in U$. Tomando $m=\operatorname{máx}\left\{m_{n}, n\right\}$ se tiene que $(m, n) \in U$ y $(n, m) \in[n, \omega]^{2}$. De manera que $((n, m),(n, m)) \in A \cap V$. Como $V$ es cualquier vecindad de $(0,(\omega, \omega))$ resulta que $(0,(\omega, \omega)) \in c l_{X}(A)$.

Supongamos que $X$ es de Whyburn. Entonces existe un subconjunto $B \subset$ $A$ tal que $c l_{X}(B) \backslash A=\{(0,(\omega, \omega))\}$. Obsérvese que $\{0\}=\pi_{S_{\omega}}[\{(0,(\omega, \omega))\}] \subset$ $\pi_{S_{\omega}}\left[c l_{X}(B)\right]$ y, por continuidad, $0 \in c l_{S_{\omega}}\left(\pi_{S_{\omega}}(B)\right)$. Entonces, existe al menos una espina, digamos $r$, tal que $\pi_{S_{\omega}}(B)$ intersecta a $r$ en un conjunto infinito. De aquí $((r, m),(r, m)) \in B$ para toda $m>m_{0}$ para algún $m_{0} \in \omega$. De modo que $(r, \omega) \in \pi_{(\omega+1)^{2}} c l_{X}(B)$ implica que $(0,(r, \omega)) \in c l_{X}(B) \backslash A$. Esto muestra que $X$ no es Whyburn.

2.61 Ejemplo ([17]). Sea $L=D \cup\{\infty\}$ la lindelöficación en un punto de un espacio discreto $D$ de cardinalidad $\omega_{1}$ y sea $I=[0,1]$ el intervalo compacto. Entonces el espacio $X=L \times I$ no es débilmente Whyburn.

Demostración. Sean $f: D \longrightarrow I$ una función inyectiva y $A \subset X$ la gráfica de $f, A=\left\{(\alpha, f(\alpha)): \alpha<\omega_{1}\right\}$. Verifiquemos que $A$ no es cerrado en $X$. En efecto, sea $x \in I$ un punto de acumulación completa del conjunto $f(D) \subset I$, es decir, $|f(D) \cap U|=|f(D)|$ es no numerable para cada vecindad $U$ de $x$. Entonces para cualquier vecindad $W$ de $(\infty, x)$ se tiene que $W \cap(A \backslash$ $\{(\infty, x)\}) \neq \emptyset$. Por tanto, $(\infty, x)$ es un punto de acumulación de $A$ en $X$, esto es, $(\infty, x) \in \operatorname{cl}_{X}(A) \backslash A$. De aquí, $A$ no es cerrado en $X$.

Mostremos que $A$ sirve para verificar que $X$ no es débilmente Whyburn. Sea $F \subset A$ un conjunto tal que $c l_{X}(F) \backslash A \neq \emptyset$. Entonces $|F|=\omega_{1}$, ya que si $|F|=\omega$ y $\alpha=\sup \{\beta:(\beta, f(\beta)) \in F\}$, entonces $\left(\left(\alpha, \omega_{1}\right) \cup\{\infty\}\right) \times[0,1]$ es una vecindad de $\{\infty\} \times[0,1]$, así que $F$ no tiene punto de acumulación en $X$, lo que es una contradicción. Luego, $G=\pi_{I}(F)$ es un subconjunto de cardinalidad $\omega_{1}$ del conjunto compacto $I$. Supongamos que $x$ es el único punto de acumulación completo de $G$. Para cada $y \in I$ distinto de $x$, existe una vecindad $V_{y}$ de $y$ tal que $\left|V_{y} \cap G\right| \leq \omega$. Entonces $\left\{V_{y}: y \neq x, y \in I\right\}$ cubre a $I \backslash\{x\}$ y, puesto que $I \backslash\{x\}$ es de Lindelöf, existen $y_{n} \in I$ distintos 
de $x$ tales que $\left\{V_{y_{n}}: n \in \omega\right\}$ cubre a $I \backslash\{x\}$. De aquí la cardinalidad de $G$ es numerable y, por lo tanto, hay al menos dos puntos de acumulación completos en $G$, lo que significa que $X$ no es débilmente Whyburn.

Por el Corolario 2.54, se sigue que $Y=\left(\omega_{1}+1\right) \times[0,1]$ es débilmente Whyburn. Sin embargo, por ejemplo se tiene que $X=L \times[0,1] \subset Y$ no es un subespacio débilmente Whyburn.

\subsection{Propiedad de Whyburn y Estrechez de Abanico}

Este pequeña sección requiere de Monoliticidad, véase 1.5.

2.62 Teorema ([7]). Cualquier espacio de Hausdorff y $\omega$ - $\psi$-monolítico con estrechez de abanico numerable es Whyburn.

Demostración. Sean $X$ un espacio $\omega-\psi-$ monolítico con estrechez de abanico numerable, $A \subset X$ y $x \in c l_{X}(A) \backslash A$. Dado que $t(X)=\aleph_{0}$, podemos suponer que $A$ es numerable. Puesto que $X$ es $\omega-\psi$-monolítico, $\psi\left(x, c l_{X}(A)\right) \leq \omega$. De manera que, existe una familia $\left\{W_{n}: n \in \omega\right\}$ decreciente y numerable de vecindades de $x$ en $c l_{X}(A)$ tal que $\bigcap\left\{c l_{X}\left(W_{n}\right): n \in \omega\right\}=\{x\}$. Luego, $x \in c l_{X}\left(W_{n} \cap A\right)$. Como $X$ tiene estrechez de abanico numerable, existen conjuntos finitos $F_{n} \subset\left(W_{n} \cap A\right)$ tales que $x \in c l_{X}\left(\bigcup_{n \in \omega} F_{n}\right)$.

Sea $F=\bigcup_{n \in \omega} F_{n}$. Entonces para cada $n \in \omega, F \backslash W_{n} \subset F_{n-1}$ es finito por definición de los $F_{n}$. Ahora, si consideramos $G_{n}=F \backslash \bigcup_{j=1}^{n-1} F_{j}$ entonces $G_{n} \subset$ $W_{n}$ y $\bigcap_{n \in \omega} c l_{X}\left(G_{n}\right) \subset \bigcap_{n \in \omega} c l_{X}\left(W_{n}\right)=\{x\}$. Pero como por la construcción $F$ y $G_{n}$ tienen los mismos puntos de acumulación y, $\bigcap_{n \in \omega} c l_{X}\left(G_{n}\right)$ sólo tiene a $x$ como punto de acumulación, resulta que $c l_{X}(F) \backslash A=\{x\}$. Por tanto, $X$ es Whyburn.

2.63 Corolario ([7]). Cualquier espacio con pseudocarácter numerable y estrechez de abanico numerable es Whyburn.

Demostración. Es consecuencia de la Proposición 1.10 y del Teorema 2.62.

2.64 Corolario ([7]). Cualquier espacio $\omega$-monolítico con estrechez de abanico numerable es Whyburn.

Demostración. Se sigue de la Proposición 1.38 y del Teorema 2.62. 



\section{Capítulo 3}

\section{Espacios $C_{p}(X)$}

Los espacios a considerar en este capítulo son de Tychonoff. Dado un espacio $X$, la familia $\tau(X)$ es una topología y $\tau^{*}(X)=\tau(X) \backslash\{\emptyset\}$.

Sea $X$ cualquier espacio topológico. Entonces $C(X)$ es el conjunto de todas las funciones continuas de $X$ en $\mathbb{R}$. Para $k \in \mathbb{N}, x_{1}, x_{2}, \ldots, x_{k} \in X$, $f \in C(X)$ y $\varepsilon>0$ sea

$$
\left[f, x_{1}, x_{2}, \ldots, x_{k}, \varepsilon\right]=\left\{g \in C(X):\left|g\left(x_{i}\right)-f\left(x_{i}\right)\right|<\varepsilon, i=1, \ldots, k\right\} .
$$

La familia de todos los conjuntos de la forma $\left[f, x_{1}, x_{2}, \ldots, x_{k}, \varepsilon\right]$ es una base para la topología en $C_{p}(X)$ llamada la topología de la convergencia puntual.

3.1 Definición. $C_{p}(X)$ es el conjunto de las funciones continuas de valores reales de $X$ en $\mathbb{R}$ con la topología de la convergencia puntual. Esto significa que $C_{p}(X)$ tiene la topología de subespacio inducida por $\mathbb{R}^{X}$.

3.2 Proposición. $C_{p}(X)$ es denso en $\mathbb{R}^{X}$.

Demostración. Como $C_{p}(X)$ es un subespacio del espacio $\mathbb{R}^{X}$, basta verificar que $\mathbb{R}^{X} \subseteq c l_{\mathbb{R}^{X}}\left(C_{p}(X)\right)$. Sea $f \in \mathbb{R}^{X}$. Para cualquier conjunto finito $x_{1}, \ldots, x_{k} \in X$ existe una función $g \in C_{p}(X)$ tal que $g\left(x_{i}\right)=f\left(x_{i}\right)$ con $i=1, \ldots, k$, lo que implica que $f \in c l_{\mathbb{R}^{X}}\left(C_{p}(X)\right)$.

3.3 Proposición. $C_{p}(X)$ es un grupo topológico respecto a la operación de adición. 
3.4 Proposición ([21]). Si $C_{p}(X)$ es un espacio Whyburn, entonces cualquier familia discreta $\gamma \subset \tau^{*}(X)$ es numerable.

Demostración. Supongamos que existe una familia discreta $\gamma=\left\{U_{\alpha}: \alpha<\right.$ $\left.\omega_{1}\right\}$ de subconjuntos abiertos y no vacíos de $X$. Fijemos un punto $x_{\alpha} \in U_{\alpha}$ para cada $\alpha<\omega_{1}$ y elegimos una función $f_{\alpha} \in C_{p}(X)$ tal que $f_{\alpha}\left(x_{\alpha}\right)=1$ y $\left.f_{\alpha}\right|_{\left(X \backslash U_{\alpha}\right)} \equiv 0$. Dada cualquier función $f: A \rightarrow \mathbb{R}$ donde $A=\left\{x_{\alpha}: \alpha<\omega_{1}\right\}$, sea $\varphi(f)(x)=\sum\left\{f\left(x_{\alpha}\right) \cdot f_{\alpha}(x): \alpha<\omega_{1}\right\}$ para cualquier $x \in X$.

Por definición de $\varphi(f)(x)$, como la familia $\gamma$ es discreta y cada $x_{\alpha} \in U_{\alpha}$, se tiene que $A$ es discreto y, en tal caso, cualquier función de $A$ en $\mathbb{R}$ es continua. Sean $x \in X$ y $U$ una vecindad de $x$ en $X$. Como $\gamma$ es discreta, $U \cap U_{\alpha_{0}} \neq \emptyset$ a lo más para algún $\alpha_{0}<\omega_{1}$. Sea $y \in U$. Entonces, $(\varphi(f))(y)=\sum_{\alpha<\omega_{1}} f\left(x_{\alpha}\right)$. $f_{\alpha}(y)$. Luego, si $y \in U$ se tiene que $y \notin U_{\beta}$ para toda $\beta \neq \alpha_{0}$, esto es, $f_{\beta}(y)=0$ para cada $\beta \neq \alpha_{0}$. Esto implica que $\varphi(f)(y)=f\left(x_{\alpha_{0}}\right) \cdot f_{\alpha_{0}}(y)$, o sea, que $\varphi(f)=f\left(x_{\alpha_{0}}\right) \cdot f_{\alpha_{0}}$ es continua, debido a que es el producto de una función constante por una función continua. De modo que, $\varphi(f) \in C_{p}(X)$.

Sean $f, g \in \mathbb{R}^{A}, f \neq g$. Entonces existe $x_{\alpha} \in A$ tal que $f\left(x_{\alpha}\right) \neq g\left(x_{\alpha}\right)$, de manera que $\varphi(f)\left(x_{\alpha}\right) \neq \varphi(g)\left(x_{\alpha}\right)$. Por tanto, $\varphi(f) \neq \varphi(g)$, es decir, la función $\varphi: \mathbb{R}^{A} \rightarrow \varphi\left(\mathbb{R}^{A}\right)$ es biyectiva. La función inversa $\varphi^{-1}$ es continua, pues para cualquier vecindad $U=\left[\varphi(f), f\left(x_{1}\right), \ldots, f\left(x_{k}\right), \varepsilon\right]$ en $C_{p}(X)$ se tiene que $\varphi^{-1}\left(U \cap \varphi\left(\mathbb{R}^{A}\right)\right) \subset\left[f, x_{1}, \ldots, x_{k}, \varepsilon\right]$. Por tanto, $\varphi$ es una inmersión. Como consecuencia, $\mathbb{R}^{\omega_{1}}$ se encaja en $C_{p}(X)$ y, por la Proposición 2.5, es un espacio Whyburn. Esto contradice que el espacio $\omega_{1}+1$ que no es Whyburn se encaja en $\mathbb{R}^{\omega_{1}}$.

3.5 Lema ([1]). Para cada familia localmente finita de conjuntos abiertos no vacíos de cardinalidad $\kappa$ en $(X, \tau)$, existe una familia de cardinalidad $\kappa$ localmente finita de conjuntos abiertos no vacíos y mutuamente ajenos.

Demostración. Sea $\mathcal{U}=\left\{U_{\alpha}: \alpha \in \kappa\right\}$ una familia localmente finita de conjuntos abiertos no vacíos. Elegimos un conjunto abierto no vacío $V_{0} \subseteq$ $U_{0}$ que intersecte sólo a una cantidad finita de elementos de $\mathcal{U}$, digamos $\mathcal{U}_{0}=\left\{U \in \mathcal{U}: U \cap V_{0} \neq \emptyset\right\}$. Una vez que se escogen subfamilias finitas $\mathcal{U}_{\beta}$ y conjuntos abiertos disjuntos $V_{\beta}$ para cada $\beta \in \alpha<\kappa$, obsérvese que $\left|\bigcup\left\{\mathcal{U}_{\beta}: \beta \in \alpha\right\}\right|<|\mathcal{U}|$ y sea $\gamma_{\alpha}=\min \left\{\eta: U_{\eta} \notin \bigcup\left\{\mathcal{U}_{\beta}: \beta \in \alpha\right\}\right\}$. Entonces $U_{\gamma_{\alpha}} \cap V_{\beta}=\emptyset$ para cada $\beta \in \alpha$. Sea $V_{\alpha}$ un conjunto abierto contenido en $U_{\gamma_{\alpha}}$ que intersecta a sólo un número finito de elementos de $\mathcal{U}$ y sea $U_{\alpha} \subset \mathcal{U}$ la familia finita de todos los elementos de $\mathcal{U}$ que intersectan a $V_{\alpha}$. De este modo, 
se obtiene una familia localmente finita de cardinalidad $\kappa,\left\{V_{\alpha}: \alpha \in \kappa\right\}$ de abiertos mutuamente disjuntos.

3.6 Corolario. En un espacio regular $X$, si existe una familia $\mathcal{U}$ de abiertos de cardinalidad $\kappa$ y localmente finita, entonces existe una familia discreta de cardinalidad $\kappa$ de abiertos que es un refinamiento de $\mathcal{U}$.

Demostración. Sea $\mathcal{U}=\left\{U_{\gamma}: \gamma \in \kappa\right\}$ una familia localmente finita de abiertos de cardinalidad $\kappa$. Por el Lema 3.5, existe una familia $\mathcal{V}=\left\{V_{\alpha}: \alpha \in \kappa\right\}$ de cardinalidad $\kappa$ localmente finita de conjuntos abiertos no vacíos y mutuamente ajenos que refina a $\mathcal{U}$. Por regularidad, para cada $\alpha \in \kappa$, existe un $W_{\alpha} \in \tau(X)$ tal que $W_{\alpha} \subset c l_{X}\left(W_{\alpha}\right) \subset V_{\alpha}$. Por tanto, $\left\{W_{\alpha}: \alpha \in \kappa\right\}$ es una familia discreta de abiertos que es un refinamiento de $\mathcal{U}$.

3.7 Corolario ([21]). Si $X$ es paracompacto y $C_{p}(X)$ es un espacio Whyburn, entonces $X$ es de Lindelöf.

Demostración. Sea $\mathcal{U}$ una cubierta abierta de $X$. Por la paracompacidad de $X$, la familia $\mathcal{U}$ tiene un refinamiento abierto localmente finito $\mathcal{V}$ de cardinalidad $\kappa$. Como consecuencia del Corolario 3.6, existe en $X$ una familia discreta $\mathcal{W}$ de abiertos con $|\mathcal{W}|=\kappa$ y, por el Lema 3.4, resulta que $|\mathcal{W}|=\omega$, o sea $\mathcal{V}=\left\{V_{n}: n \in \omega\right\}$. Luego, para cada $n \in \omega$ escogemos $\alpha_{n}$ tal que $V_{n} \subseteq U_{\alpha_{n}}$ con $U_{\alpha_{n}} \in \mathcal{U}$, de manera que $\left\{U_{\alpha_{n}}: n \in \omega\right\}$ es la subcubierta numerable.

Se dice que $\gamma$ es una $\omega$-cubierta de un espacio $X$, si dado cualquier subconjunto finito de $X$ existe un elemento de la cubierta que lo contiene.

3.8 Lema ([21]). Sean $X$ un espacio normal y $C_{p}(X)$ de Whyburn. Supongamos que tenemos una sucesión $\left\{\gamma_{n}: n \in \omega\right\}$ de cubiertas abiertas de $X$ con las siguientes propiedades:

1. $\gamma_{n}=\left\{U_{m}^{n}: m \in \omega\right\}$ y $U_{m}^{n} \subset U_{m+1}^{n}$ para cada $m \in \omega$;

2. Para cada $n \in \omega$ existe una cubierta cerrada $\mu_{n}=\left\{F_{m}^{n}: m \in \omega\right\}$ de $X$ tal que $F_{m}^{n} \subset U_{m}^{n}$ y $F_{m}^{n} \subset F_{m+1}^{n}$ para todo $m \in \omega$.

Entonces es posible elegir $W_{n} \in \gamma_{n}$ para cada $n \in \omega$ tal que $\left\{W_{n}: n \in \omega\right\}$ es una $\omega$-cubierta de $X$. 
Demostración. Para cada par $(m, n)$ de números naturales, podemos elegir por normalidad un $f_{m}^{n} \in C_{p}(X)$ tal que $\left.f_{m}^{n}\right|_{F_{m}^{n}} \equiv \frac{1}{n}$ y $\left.f_{m}^{n}\right|_{X \backslash U_{m}^{n}} \equiv 1$. Luego, la sucesión $S_{n}=\left\{f_{m}^{n}: m \in \omega\right\}$ converge en $C_{p}(X)$ a la función $h_{n} \equiv \frac{1}{n}$, o sea $\operatorname{lím}_{m \rightarrow \infty} f_{m}^{n}(x)=\frac{1}{n}$ para cada $x \in X$ debido a que los $F_{m}^{n}$ forman una cubierta ascendente por definición. En consecuencia, la función $h \equiv 0$ está en la cerradura del conjunto $S=\bigcup\left\{S_{n}: n \in \omega\right\}$.

Aplicando la propiedad de Whyburn del espacio $C_{p}(X)$, encontramos un conjunto casi cerrado $F \subset S$ tal que $h \in c_{C_{p}(X)}(F)$. Observe que para cualquier natural $n$, el conjunto $F_{n}=F \cap S_{n}$ no puede ser infinito porque de otro modo para dicho $n$ habría una infinidad de $m$ 's tales que $f_{m}^{n} \in F$, lo cual implica que $h_{n} \in c l_{X}(F) \backslash F$ contradiciendo el hecho de que $F$ es casi cerrado. Por lo tanto, para cada $n \in \omega$ tenemos un número natural $m(n)$ tal que $F_{n} \subset\left\{f_{m}^{n}: m \leq m(n)\right\}$.

Para cada $n \in \omega$ sea $W_{n}=U_{m(n)}^{n}$ y sea $K$ un subconjunto finito de $X$. Como $h \in c l_{X}(F)$, existe $f_{m}^{n} \in F$ tal que $f_{m}^{n}(x)<1$ para todo $x \in K$. Así que, $K \cap\left(X \backslash U_{m}^{n}\right)=\emptyset$, lo que implica que $K \subset U_{m}^{n}$. Luego, como $m \leq m(n)$ se tiene que $K \subset U_{m}^{n} \subset U_{m(n)}^{n}=W_{n}$. Por tanto, $\left\{W_{n}: n \in \omega\right\}$ es una $\omega$-cubierta de $X$.

3.9 Lema. Sean $X$ un espacio de Lindelöf y $\mathcal{F}=\left\{F_{n}: n \in \omega\right\}$ una cubierta abierta numerable de $X$. Entonces $\mathcal{F}$ tiene un encogimiento numerable por cerrados.

Demostración. Por regularidad de $X$, para cada $x \in X$ podemos escoger $U_{x}, V_{x} \in \tau(X)$ tales que $x \in U_{x} \subseteq c l_{X}\left(U_{x}\right) \subseteq V_{x}$ donde $V_{x}$ está contenido en algún elemento de $\mathcal{F}$. Entonces $\left\{U_{x}: x \in X\right\}$ tiene una subcubierta abierta numerable $\mathcal{U}=\left\{U_{\beta}: \beta \in J\right\}$ donde $J$ es numerable, debido a que $X$ un espacio de Lindelöf. Por ([11], Teorema 3.8.11), $\mathcal{U}$ tiene un refinamiento abierto localmente finito $\left\{W_{\alpha}: \alpha \in I\right\}$, donde podemos suponer que $I$ es numerable. De manera que, $\left\{c l_{X}\left(W_{\alpha}\right): \alpha \in I\right\}$ es un refinamiento localmente finito por cerrados de $\mathcal{F}$. Para cada $\alpha \in I$, sean $A_{n}=\left\{\alpha \in I: c l_{X}\left(W_{\alpha}\right) \subseteq F_{n}\right\}$ y $G_{n}=\bigcup_{\alpha \in A_{n}} c l_{X}\left(W_{\alpha}\right) \subseteq F_{n}$; se tiene que $G_{n}$ es cerrado por la finitud local y por tanto, se tiene el encogimiento requerido tal que $\bigcup\left\{G_{n}: n \in \omega\right\}=$ $\bigcup U_{n}=X$. 
3.10 Definición. Se dice que $X$ es un espacio de Hurewicz si para cualquier sucesión $\left\{\gamma_{n}: n \in \omega\right\}$ de cubiertas abiertas de $X$, existe para cada $n \in \omega$ una subfamilia finita $\mu_{n} \subset \gamma_{n}$ tal que $\bigcup\left\{\mu_{n}: n \in \omega\right\}$ es una cubierta de $X$.

3.11 Teorema ([21]). Supongamos que $C_{p}(X)$ es un espacio Whyburn y $X$ es paracompacto. Entonces $X$ es un espacio de Hurewicz.

Demostración. Del Corolario 3.7 se sigue que $X$ es un espacio de Lindelöf. Sea $\left\{\lambda_{n}: n \in \omega\right\}$ una sucesión de cubiertas abiertas del espacio $X$. Como $X$ es de Lindelöf podemos suponer sin pérdida de generalidad que cada $\lambda_{n}$ es numerable y sea $\left\{W_{m}^{n}: m \in \omega\right\}$ una enumeración de $\lambda_{n}$ para cada $n$. Definimos $U_{m}^{n}=\bigcup\left\{W_{i}^{n}: i \leq m\right\}$ para cada $m, n \in \omega$. Por construcción, la familia $\gamma_{n}=\left\{U_{m}^{n}: m \in \omega\right\}$ es una cubierta abierta numerable de $X$ tal que $U_{m}^{n} \subset U_{m+1}^{n}$ para cada $m, n \in \omega$.

Por el Lema 3.9, existe una cubierta por cerrados $\left\{G_{m}^{n}: m \in \omega\right\}$ de la cubierta $\lambda_{n}$ tal que $G_{m}^{n} \subset W_{m}^{n}$ para toda $m, n \in \omega$. Ahora, si $F_{m}^{n}=\bigcup\left\{G_{i}^{n}\right.$ : $i \leq m\}$, entonces las cubiertas $\gamma_{n}=\left\{U_{m}^{n}: m \in \omega\right\}$ y $\mu_{n}=\left\{F_{m}^{n}: m \in \omega\right\}$ satisfacen las hipótesis del Lema 3.8. De modo que podemos elegir $W_{n} \in \gamma_{n}$ tal que $\left\{W_{n}: n \in \omega\right\}$ es una cubierta de $X$. Luego, como cada $W_{n}$ es la unión de una cantidad finita de elementos de $\lambda_{n}$, existen familias $\nu_{n} \subset \lambda_{n}$ tales que $\bigcup\left\{\bigcup \nu_{n}: n \in \omega\right\}=X$. Por lo tanto, $X$ es un espacio de Hurewicz.

3.12 Corolario ([21]). Si $X$ es un espacio metrizable y $C_{p}(X)$ es Whyburn, entonces $X$ es separable y tiene la propiedad de Hurewicz.

Demostración. Por el Corolario 3.7, $X$ es de Lindelöf y por ser metrizable es separable. Por el Teorema 3.11, $X$ es de Hurewicz.

3.13 Corolario ([21]). Si $\mathbb{P}$ es el espacio de los números irracionales con su topología natural, entonces $C_{p}(\mathbb{P})$ no es Whyburn.

Demostración. En ([11], 4.3.G) puede encontrarse que $\mathbb{P} \cong \mathbb{N}^{\omega}=\prod_{i \in \mathbb{N}} \mathbb{N}_{i}$. Sea $\left\{\gamma_{m}: m \in \omega\right\}$ una sucesión de cubiertas abiertas de $\prod_{i \in \mathbb{N}} \mathbb{N}_{i}$, donde $\gamma_{m}=$ $\left\{\{0,1, \ldots, n\} \times \prod_{i \neq m} \mathbb{N}_{i}: n \in \mathbb{N}\right\}$. Afirmamos que no existe una subfamilia $\mu_{m} \subseteq \gamma_{m}$ para cada $m \in \omega$ tal que $\bigcup \mu_{m}$ sea una cubierta de $X$. Supongamos lo contrario, entonces cada $\mu_{m}$ tiene un elemento mayor y ese elemento mayor es de la forma $\bigcup \mu_{m}=\left\{0,1, \ldots, n_{m}\right\} \times \Pi_{i \neq m} \mathbb{N}_{i}$ para $n_{m} \in \omega$. Definimos 
$f \in \mathbb{N}^{\omega}$ por $f(m)=n_{m}+1$ para cada $m \in \mathbb{N}$. Obsérvese que $f \notin \bigcup \mu_{m}$ para cada $m \in \mathbb{N}$, es decir, $f$ no pertenece a $\bigcup\left\{\bigcup \mu_{m}: m \in \omega\right\}$. Por lo tanto, $\left\{\bigcup \mu_{m}: m \in \omega\right\}$ no es una cubierta de $\mathbb{N}^{\omega}$, por lo que $\mathbb{P}$ no es un espacio de Hurewicz y, en consecuencia, $C_{p}(\mathbb{P})$ no es Whyburn.

3.14 Teorema ([21]). Sea $C_{p}(X)$ un espacio Whyburn con estrechez numerable. Entonces $C_{p}(X)$ es discretamente generado (ver 1.25).

Demostración. Fijemos cualesquiera $A \subset C_{p}(X)$ y $f \in c l_{X}(A)$. Como $C_{p}(X)$ es un grupo topológico respecto a la operación de adición, es un espacio homogéneo. Entonces, podemos suponer sin pérdida de generalidad que $f \equiv \overline{0}$ y que $f \in \mathrm{cl}_{C_{p}(X)}(A) \backslash A$. Como $C_{p}(X)$ es de Whyburn y tiene estrechez numerable, podemos restringirnos al caso cuando $A$ es numerable y casi cerrado. Sea $\left\{f_{n}: n \in \omega\right\}$ alguna enumeración de $A$. Luego, existe una sucesión $\left\{\varepsilon_{n}: n \in \omega\right\}$ de reales positivos tal que $\varepsilon_{n} \rightarrow 0$ y $f_{i}+\varepsilon_{i} \neq f_{j}, f_{i}+\varepsilon_{i} \neq \overline{0}$ para cualesquiera $i, j \in \omega$. Consideremos el conjunto $B=\left\{g_{n}: n \in \omega\right\}$ donde $g_{n}=f_{n}+\varepsilon_{n}$ para cada $n \in \omega$.

Afirmamos que $\overline{0} \in c l_{C_{p}(X)}(B)$. En efecto, sea $U=\left[\overline{0}, x_{1}, \ldots, x_{l}, \varepsilon\right]=$ $\left\{g \in C_{p}(X):\left|g\left(x_{i}\right)-\overline{0}\left(x_{i}\right)\right|<\varepsilon\right.$ para cada $\left.i \leq l\right\}$ una vecindad básica de $\overline{0}$. Como $\overline{0} \in \operatorname{cl}_{C_{p}(X)}(A) \backslash A$, el conjunto $M=\left\{n \in \omega: f_{n} \in\left[\overline{0}, x_{1}, \ldots, x_{l}, \frac{\varepsilon}{2}\right]\right\}$ es infinito. Entonces, para cada $n \in M$ con $\varepsilon_{n}<\frac{\varepsilon}{2}$ tenemos que $\left|g_{n}\left(x_{i}\right)-\overline{0}\left(x_{i}\right)\right|$ $=\left|f_{n}\left(x_{i}\right)+\varepsilon_{n}-\overline{0}\left(x_{i}\right)\right| \leq\left|f_{n}\left(x_{i}\right)-\overline{0}\left(x_{i}\right)\right|+\varepsilon_{n}<\frac{\varepsilon}{2}+\frac{\varepsilon}{2}=\varepsilon$ para cada $i \leq l$. Por tanto, $g_{n} \in U$ y, en conclusión, $\overline{0} \in \operatorname{cl}_{C_{p}(X)}(B)$.

Como $C_{p}(X)$ es de Whyburn y $\overline{0} \in c_{C_{p}(X)}(B) \backslash B$, existe un conjunto casi cerrado $P=\left\{g_{n_{k}}: k \in \omega\right\} \subset B$ tal que $\{\overline{0}\}=c l_{C_{p}(X)}(P) \backslash P$. Veamos que el subespacio $D=\left\{f_{n_{k}}: k \in \omega\right\}$ es discreto. En efecto, para cada $k \in \omega$ tenemos que $f_{n_{k}} \notin P$ y $g_{n_{k}} \neq \overline{0}$, por la forma en que se eligieron. Por eso hay un conjunto abierto $W=\left[f_{n_{k}}, x_{1}, \ldots, x_{l}, \varepsilon\right]$ tal que $W \cap P=\emptyset$. Si hay una infinidad de $i \in \omega$ tales que $f_{n_{i}} \in V=\left[f_{n_{k}}, x_{1}, \ldots, x_{l}, \frac{\varepsilon}{2}\right]$ entonces existe un número natural $j \neq k$ tal que $\varepsilon_{n_{j}}<\frac{\varepsilon}{2} \mathrm{y}$, en consecuecia se tiene que, $\left|g_{n_{j}}\left(x_{p}\right)-f_{n_{k}}\left(x_{p}\right)\right| \leq\left|f_{n_{j}}\left(x_{p}\right)-f_{n_{k}}\left(x_{p}\right)\right|+\varepsilon_{n_{j}}<\frac{\varepsilon}{2}+\frac{\varepsilon}{2}=\varepsilon$ para cada $p \leq l$ lo que implica que $g_{n_{j}} \in W \cap P$, lo que es una contradicción. Así, $V$ es una vecindad de $f_{n_{k}}$ que intersecta sólo a una cantidad finita de elementos de $D$. Por tanto $D$ es discreto.

Falta probar que $\overline{0} \in \mathrm{cl}_{C_{p}(X)}(D)$. Sea $U=\left[\overline{0}, x_{1}, \ldots, x_{l}, \varepsilon\right]$ una vecindad básica de $\overline{0}$. Como $\overline{0} \in c_{C_{p}(X)}(P) \backslash P$, el conjunto $N=\left\{k \in \omega: g_{n_{k}} \in\right.$ 
$\left.\left[\overline{0}, x_{1}, \ldots x_{l}, \frac{\varepsilon}{2}\right]\right\}$ es infinito. Elegimos cualquier $k \in N$ tal que $\varepsilon_{n_{k}}<\frac{\varepsilon}{2}$. Entonces $\left|f_{n_{k}}\left(x_{i}\right)-\overline{0}\left(x_{i}\right)\right| \leq\left|g_{n_{k}}\left(x_{i}\right)-\overline{0}\left(x_{i}\right)\right|+\varepsilon_{n_{k}}<\frac{\varepsilon}{2}$ para toda $i \leq l$, lo que muestra que $f_{n_{k}} \in U$, es decir, $\overline{0} \in c_{C_{p}(X)}(D)$. Por tanto, $C_{p}(X)$ es discretamente generado.

\section{Una caracterización para que $C_{p}(\xi)$ sea débil- mente Whyburn}

A continuación se da una caracterización para aquellos ordinales $\xi$ (con la topología del orden) para los cuales $C_{p}(\xi)$ es débilmente Whyburn, pero antes necesitamos las siguientes definiciones y resultados previos.

3.15 Definición. Un cardinal $\kappa$ es $\omega$-inaccesible siempre que $\lambda^{\omega}<\kappa$ para cualquier cardinal $\lambda<\kappa$.

El siguiente resultado es una elegante caracterización para que un espacio $C_{p}(\xi)$ sea pseudoradial para un ordinal $\xi$ (ver [13], Teorema 3).

3.16 Teorema $([13]) . C_{p}(\xi)$ es pseudoradial si y sólo si $\operatorname{cf}(\xi) \leq \omega$ o bien $\xi$ es regular y $\omega$-inaccesible.

Se requiere del siguiente Teorema para mostrar el Teorema 3.20 porque esencialmente muestra la equivalencia entre la propiedad de Fréchet-Urysohn y la radialidad en espacios de funciones (Ver [13], Teoremas 2 y 3).

3.17 Teorema ([13]). Las siguientes condiciones son equivalentes para cualquier ordinal $\xi$ :

1. $C_{p}(\xi)$ es de Fréchet-Urysohn.

2. $C_{p}(\xi)$ es radial.

3. $c f(\xi) \leq \omega$. 
3.18 Lema $([9])$. Sea $B \subseteq C_{p}(\xi), c f(\xi)=\kappa$ con $|B|<\kappa$. Si $g \in c l_{X}(B) \backslash B$ entonces existe una sucesión $S \subseteq B$ que converge a $g$.

Demostración. Si $\kappa=\omega$, no hay nada que mostrar porque $B$ es cerrado. Entonces supongamos que $\kappa>\omega$. Dado que $C_{p}(\xi)$ es un grupo topológico, es homogéneo, podemos suponer sin pérdida de generalidad que $g=\overline{0}$, donde $\overline{0}$ denotará la función constante igual a cero. Cada $f \in B$ es constante en una cola de $\xi$, digamos que $f$ es constante en $\left(\eta_{f}, \xi\right)$. Sea $\eta=\sup \left\{\eta_{f}: f \in B\right\}$. Entonces $\eta<\xi$, es decir, todas las funciones $f \in B$ son constantes en $[\eta, \xi)$. Sea $\alpha>\eta$ y definimos $B_{\alpha}=\left\{\left.f\right|_{\alpha+1}: f \in B\right\}$. Puesto que $c f(\alpha+1)=1 \leq \omega$, tenemos que $C_{p}(\alpha+1)$ es Fréchet, por el Teorema 3.17. Entonces existe una sucesión $\left\{f_{n}\right\}_{n \in \omega} \subseteq B_{\alpha}$ que converge a $\overline{0}_{\alpha+1}$. Sea $f_{n}(\eta)=r_{n}$. Como $\alpha>\eta$, se tiene que $\left\{f_{n}(\eta)\right\}_{n \in \omega}$ converge a 0 , de manera que $r_{n} \rightarrow 0 \mathrm{y}$, por tanto, $\left\{f_{n}\right\}_{n \in \omega}$ converge a $\overline{0}$.

El siguiente resultado es una consecuencia del Teorema del $\Delta$-sistema (ver Teorema 1.27)

3.19 Lema ([9]). Supongamos que $\kappa$ es regular y $\omega$-inaccesible. Para cada $\alpha \in \kappa$ e $i \in \omega$, sea $A_{\alpha}^{i}$ un conjunto numerable. Entonces, existen $D \in[\kappa]^{\kappa} y$ $R_{i}$ con $i \in \omega$ tal que para cada $i \in \omega, A_{\alpha}^{i} \cap A_{\alpha^{\prime}}^{i}=R_{i}$ para $\alpha, \alpha^{\prime} \in D$ distintos.

Demostración. Para cualquier $\alpha$, sea $A_{\alpha}=\bigcup\left\{A_{\alpha}^{i} \times\{i\}: i \in \omega\right\}$. Aplicando el Teorema 1.27 a la familia $\mathcal{A}=\left\{A_{\alpha}: \alpha \in \kappa\right\}$, vemos que existen $\mathcal{A}^{\prime}=$ $\left\{A_{\alpha}: \alpha \in D \in[\kappa]^{\kappa}\right\} \subset \mathcal{A}$ con $\left|\mathcal{A}^{\prime}\right|=\kappa$ y $R$ tales que para cualesquiera $A_{\alpha}, A_{\alpha^{\prime}} \in \mathcal{A}^{\prime}$ distintos se tiene que $A_{\alpha} \cap A_{\alpha^{\prime}}=R$. Sea $R_{i}=\{x:(x, i) \in R\}$. Dado $i \in \omega$, sucede que $(z, i) \in A_{\alpha} \cap A_{\alpha^{\prime}}=R$ siempre que $z \in A_{\alpha}^{i} \cap A_{\alpha^{\prime}}^{i}=R_{i}$, lo que termina la prueba.

Sean $\xi$ un ordinal, $\varepsilon>0$ y $f \in C_{p}(\xi)$. Denotamos

$$
\begin{aligned}
& J(f, \varepsilon)=\{\alpha \in \xi:|f(\alpha)-f(\alpha+1)|>\varepsilon\}, \\
& J(f)=\bigcup_{\varepsilon>0} J(f, \varepsilon) .
\end{aligned}
$$

Dicho de otro modo, si $f(\alpha) \neq f(\alpha+1)$ entonces existe $\varepsilon>0$ tal que $|f(\alpha)-f(\alpha+1)|>\varepsilon$, lo que implica que $\alpha \in J(f, \varepsilon)$ y, en consecuencia, $\alpha \in$ $J(f)$. Además, para $f \in C_{p}(\xi)$ y $\alpha \in J(f)$, definimos $\delta_{f}(\alpha)=f(\alpha+1)-f(\alpha)$. 
Obsérvese que $J(f, \varepsilon)$ es finito si $c f(\xi) \neq \omega$. Para verificarlo, nótese que $\xi$ es numerablemente compacto y supongamos que $|J(f, \varepsilon)| \geq \omega$. Sea $\alpha \in \xi$ el mínimo punto de acumulación de $J(f, \varepsilon)$. Entonces existe una sucesión $\left\{\alpha_{n}: n \in \omega\right\} \subseteq J(f, \varepsilon)$ que converge a $\alpha$. No obstante, por definición de $J(f, \varepsilon)$ se tiene que $\left|f\left(\alpha_{i}\right)-f\left(\alpha_{i}+1\right)\right|>\varepsilon$, lo que implica que la función $f$ no es continua en $\alpha$, lo que no puede suceder. Así, $J(f, \varepsilon)$ es finito.

Por otro lado, $J(f, \varepsilon)$ es a lo más numerable si $c f(\xi)=\omega$. Supongamos que $|J(f, \varepsilon)| \geq \omega_{1}$ y sea $\lambda$ un punto de $\omega_{1}$-acumulación de $J(f, \varepsilon)$. Como $c f(\xi)=\omega$ se tiene que $\xi$ es de Lindelöf, de modo que existe $\left\{\lambda_{n}: n \in \omega\right\} \subseteq \xi$ tal que $\xi=\sup \left(\lambda_{n}\right)$. Luego, para alguna $n \in \omega$ resulta que $\lambda \in J(f, \varepsilon) \cap \lambda_{n}$. Prosiguiendo como en el párrafo anterior, se sigue que $J(f, \varepsilon) \cap \lambda_{n}$ es finito, de donde, $J(f, \varepsilon)$ es a lo más numerable.

Por lo tanto, $J(f)$ es numerable para cualquier $\xi$.

Una función $f \in C_{p}(\xi)$ está determinada de manera única por los siguientes objetos:

(1) el valor $f(0)$,

(2) el conjunto (numerable) $J(f)$,

(3) la función $\delta_{f}: J(f) \rightarrow \mathbb{R}$.

Obsérvese que los ordinales $\alpha<\xi$ determinan los valores de $f$. En efecto, procediendo por inducción, por (1), sabemos que $f(0)$ está determinada de manera única. Ahora, supongamos que $f(\beta)$ está únicamente determinada para toda $\beta<\alpha$. Luego, definimos $f(\alpha)$ como sigue:

1. Si $\alpha$ no es límite, $\alpha=\gamma+1$.

a) Si $\gamma \neq J(f)$ entonces $f(\alpha)=f(\gamma)$.

b) Si $\gamma \in J(f)$ entonces $f(\alpha)=f(\gamma)+\delta_{f}(\gamma)$.

2. Si $\alpha$ es límite, $f(\alpha)$ está determinado por la continuidad de $f$. 
3.20 Teorema ([9]). Sea $\xi$ un número ordinal con $c f(\xi)=\kappa$. Entonces $C_{p}(\xi)$ es débilmente Whyburn si y sólo si $\kappa \leq \omega$ o bien $\kappa$ es $\omega$-inaccesible.

Demostración. Por el Teorema 3.17, si $c f(\xi) \leq \omega$ entonces $C_{p}(\xi)$ es de Fréchet-Urysohn y, de la Proposición 2.4, $C_{p}(\xi)$ es Whyburn. Entonces supongamos que $c f(\xi)=\kappa>\omega$. Probaremos dos implicaciones:

1) Si $\kappa$ es $\omega$-accesible entonces $C_{p}(\xi)$ no es débilmente Whyburn.

Sea $j: \kappa \rightarrow \xi$ una función creciente, cofinal tal que $j(0)=0$ y que preserva supremos; es decir, si $A \subset \kappa$ y $\gamma=\sup (A)$ entonces $j(\gamma)=\sup (j(A))$. Entonces $j(\kappa)$ es homeomorfo a $\kappa$. Luego, si $\beta \in \xi$, existe $\alpha \in \kappa$ tal que $\beta \in[j(\alpha), j(\alpha+1))$ y se define la retracción $r^{\prime}: \xi \longrightarrow j(\kappa)$ por $r(\beta)=j(\alpha)$.

Sea $A=j(\kappa)$. Como $A$ es un retracto de $\xi$, entonces existe $r: \xi \longrightarrow A$ tal que $r(\alpha)=\alpha$ para cada $\alpha \in A$. Definimos la función $\phi: C_{p}(A) \longrightarrow C_{p}(\xi)$ como $\phi(g)=g \circ r$. Afirmamos que la función $\phi$ es una inmersión. En efecto, sean $\phi(g)=h$ y $\left[h, \alpha_{1}, \ldots, \alpha_{k}, \varepsilon\right]$ una vecindad básica de $h$ en $C_{p}(\xi)$. Para $\beta_{i}=r\left(\alpha_{i}\right)$ con $i=1, \ldots, k$, tenemos que $\phi\left(\left[g, \beta_{1}, \ldots, \beta_{k}, \varepsilon\right]\right)=\phi\left(\left\{f: \mid f\left(\beta_{i}\right)\right.\right.$ $\left.\left.-g\left(\beta_{i}\right) \mid<\varepsilon\right\}\right)=\left\{\phi(f):\left|f\left(\beta_{i}\right)-g\left(\beta_{i}\right)\right|<\varepsilon\right\}=\left\{f \circ r:\left|f\left(\beta_{i}\right)-g\left(\beta_{i}\right)\right|<\varepsilon\right\}=$ $\left\{f \circ r:\left|f \circ r\left(\alpha_{i}\right)-g \circ r\left(\alpha_{i}\right)\right|<\varepsilon\right\}=\left\{f \circ r:\left|f \circ r\left(\alpha_{i}\right)-h\left(\alpha_{i}\right)\right|<\varepsilon\right\} \subset\{l \in$ $\left.C_{p}(\xi):\left|l\left(\alpha_{i}\right)-h\left(\alpha_{i}\right)\right|<\varepsilon\right\}=\left[h, \alpha_{1}, \ldots, \alpha_{k}, \varepsilon\right]$. Por tanto, $\phi$ es continua. Sean $g_{1}, g_{2} \in C_{p}(A)$ con $g_{1} \neq g_{2}$. Tomemos $\beta \in A$ tal que $g_{1}(\beta) \neq g_{2}(\beta)$. Para $\alpha \in$ $r^{-1}(\beta)$ tenemos que $\phi\left(g_{1}\right)(\alpha)=g_{1}(\beta) \neq g_{2}(\beta)=\phi\left(g_{2}\right)(\alpha)$, de donde, $\phi\left(g_{1}\right) \neq$ $\phi\left(g_{2}\right)$. Por tanto, $\phi: C_{p}(A) \longrightarrow \phi\left[C_{p}(A)\right]$ es biyectiva. Luego, la función inversa $\phi^{-1}$ es continua, pues para cualquier vecindad $V=\left[h, \alpha_{1}, \ldots, \alpha_{k}, \varepsilon\right]$ de $h$ en $C_{p}(\xi)$ se tiene que $\phi^{-1}\left[V \cap \phi\left(C_{p}(A)\right) \subset\left[g, r\left(\alpha_{1}\right), \ldots, r\left(\alpha_{k}\right), \varepsilon\right]\right.$, usando un argumento semejante al usado en la demostración de continuidad de $\phi$. Por tanto, $\phi$ es una inmersión. Ahora, la función $\psi: C_{p}(\xi) \longrightarrow \phi\left[C_{p}(A)\right]$ dada por $\psi(f)=\phi\left(\left.f\right|_{\kappa}\right)$ es una retracción, pues si $\varphi \in \phi\left[C_{p}(A)\right]$ entonces $\gamma=\varphi \circ r \in C_{p}(\xi)$ у $\psi(\gamma)=\phi\left(\left.\gamma\right|_{\kappa}\right)=\phi\left(\left.\varphi \circ r\right|_{\kappa}\right)=\phi(\varphi)=\gamma$ para cada $\gamma \in \phi\left[C_{p}(A)\right]$. Como $C_{p}(\xi)$ es de Hausdorff, $\phi\left[C_{p}(A)\right]$ es cerrado en $C_{p}(\xi)$ pues $\psi$ es retracción y, como $\phi\left[C_{p}(A)\right]$ es homeomorfo a $C_{p}(A)$, se sigue que $C_{p}(j(\kappa))$ es un subespacio cerrado de $C_{p}(\xi)$.

Sea $\lambda$ un cardinal que satisface que $\lambda<\kappa \leq \lambda^{\omega}$. Para cualquier $\alpha \in \kappa$ sea $f_{\alpha}: \kappa \rightarrow \mathbb{R}$ una función definida por $f_{\alpha}(\zeta)=0$ si $\zeta \leq \alpha$ y $f_{\alpha}(\zeta)=1$ si $\alpha<\zeta<\kappa$. 
Afirmación 1. El conjunto $X=\left\{f_{\alpha}: \alpha \in \kappa\right\} \cup\{\overline{0}\}$ es un subespacio cerrado de $C_{p}(\kappa)$ en que $\overline{0}$ es el único punto no aislado y para cualquier conjunto $E^{\prime} \subseteq X \backslash\{\overline{0}\}$ con $\left|E^{\prime}\right|<\kappa$ tenemos que $\overline{0} \notin c l_{X}\left(E^{\prime}\right)$.

Para cada $\alpha \in \kappa$, el conjunto abierto $\left[f_{\alpha}, \alpha, \alpha+1, \frac{1}{2}\right] \cap X=\left\{f_{\alpha}\right\}$, lo que implica que $X \backslash\{\overline{0}\}$ es discreto. De hecho, como para cualquier conjunto $E^{\prime} \subseteq X \backslash\{\overline{0}\}$ con $\left|E^{\prime}\right|<\kappa$ se tiene que para algún $\alpha_{0} \in \kappa, f(\beta)=1$ para cada $\beta \geq \alpha_{0}$, lo cual implica que $\overline{0} \notin c l_{X}\left(E^{\prime}\right)$.

Afirmación 2. El espacio $C_{p}(\lambda+1)$ tiene cardinalidad al menos $\lambda^{\omega}$.

Para cada $\alpha \in \lambda$, la función característica $\chi_{\alpha+1}$ es continua. Luego, como $\left\{\chi_{\alpha+1}: \alpha \in \lambda\right\} \subseteq C_{p}(\lambda+1)$ se sigue que $\left|C_{p}(\lambda+1)\right| \geq \lambda$. Además, por ([14], Teorema 10.10), se tiene que $\left|C_{p}(\lambda+1)\right|=\left|C_{p}(\lambda+1)\right|^{\omega}$. De manera que $\left|C_{p}(\lambda+1)\right| \geq \lambda^{\omega}$.

Afrmación 3. $C_{p}(\lambda+1)$ tiene peso $\lambda$.

Por ([4], Teorema I.1.1), se tiene que $w\left(C_{p}(\lambda+1)\right)=|\lambda+1|=\lambda$.

Afirmación 4. $C_{p}(\kappa)=C_{p}(\kappa) \times C_{p}(\lambda+1)$.

Nótese que el conjunto $\lambda+1=[0, \lambda]$ es un subespacio abierto y cerrado de $\kappa$. Además, dado que $\kappa \backslash(\lambda+1)$ tiene el mismo tipo de orden de $\kappa$ se tiene que $C_{p}(\kappa)=C_{p}([0, \lambda] \oplus[\lambda+1, \kappa))=C_{p}([0, \lambda]) \times C_{p}([\lambda+1, \kappa)) \cong C_{p}(\lambda+1) \times C_{p}(\kappa)$ por ([4], Proposición 0.34).

Afirmación 5. El espacio $X \times C_{p}(\lambda+1)$ no es débilmente Whyburn.

Como $\kappa \leq \lambda^{\omega}$, por la Afirmación 2 podemos fijar una función uno a uno $\phi: \kappa \rightarrow C_{p}(\lambda+1)$. Sea $G=\phi[\kappa]$. Considerando la Afirmación 3 y el hecho de que $G \subseteq C_{p}(\lambda+1)$ resulta que $w(G) \leq \lambda$, de donde, $L(G) \leq \lambda$. Supongamos que ninguna $f \in C_{p}(\lambda+1)$ es punto de acumulación completa de $G$. Entonces para cada $f \in C_{p}(\lambda+1)$ existe una vecindad abierta $U_{f}$ de $f$ tal que $\left|U_{f} \cap G\right|<\kappa$. De modo que $\left\{U_{f}: f \in C_{p}(\lambda+1)\right\}$ es una cubierta abierta de $G$. No obstante, como $\kappa$ es regular, no se puede cubrir a $G$ con $\lambda$ elementos de cardinalidad menor que $\kappa$. Por tanto, $G$ tiene un punto de acumulación completa, digamos z. Aplicando el mismo procedimiento a $G \backslash\{z\}$, obtenemos otro punto de acumulación completa de $G$. 
Sean $D=\left\{\left(f_{\alpha}, \phi(\alpha)\right): \alpha \in \kappa\right\} \subseteq X \times C_{p}(\lambda+1)$, z un punto de acumulación completa de $G$ y $W=U \times V$ una vecindad de $(\overline{0}, z)$. Puesto que $f_{\alpha} \rightarrow \overline{0}$, existe $\alpha_{0} \in \kappa$ tal que $f_{\alpha} \in U$ para toda $\alpha>\alpha_{0}$. Además, como $|V \cap \phi[\kappa]|=\kappa$ existe $\beta>\alpha_{0}$ tal que $\phi(\beta) \in V$, de donde, $\left(\left(f_{\beta}, \phi(\beta)\right) \in U \times V=W\right.$. Así, $(\overline{0}, z) \in c l_{X \times C_{p}(\lambda+1)}(D) \backslash D \mathrm{y}$, en consecuencia, $D$ no es cerrado.

Sea $E=\left\{\left(f_{\alpha}, \phi(\alpha)\right): \alpha \in \beta\right.$ donde $\left.\beta<\kappa\right\}$ cualquier subconjunto de $D$ tal que $c l_{X \times C_{p}(\lambda+1)}(E) \backslash D \neq \emptyset$. Como $|D|=\kappa$ se sigue que $|E| \leq \kappa$; no obstante, por la afirmación 1 , si $|E|<\kappa$ entonces $(\overline{0}, z) \notin c l_{X}(E)$ para cualquier punto de acumulación completa $z$ de $G$. De modo que $|E|=\kappa$.

Como $E_{1}=\{\phi(\alpha): \alpha \in \beta$ con $\beta<\kappa\}$ es un subconjunto de $C_{p}(\lambda+1)$ de cardinalidad $\kappa$, por la afirmación 3 se tiene que $w\left(E_{1}\right) \leq \lambda$ y, en consecuencia, $L\left(E_{1}\right) \leq \lambda$. Por el mismo argumento que se empleó en el primer párrafo de la presente afirmación, se sigue que $E_{1}$ tiene dos puntos distintos de acumulación completas $z_{1}$ y $z_{2}$. Como $\left(\overline{0}, z_{1}\right)$ y $\left(\overline{0}, z_{2}\right)$ están en $c l_{X \times C_{p}(\lambda+1)}(E)$ tenemos que $\left|c l_{X \times C_{p}(\lambda+1)}(E) \backslash D\right| \neq 1$. Por tanto, $X \times C_{p}(\lambda+1)$ no es débilmente Whyburn.

Para finalizar la prueba nótese que, por las afirmaciones 1 y 4 , el conjunto $X \times C_{p}(\lambda+1)$ es un subespacio cerrado de $C_{p}(\kappa)$ y, por tanto, de $C_{p}(\xi)$. Finalmente, por la Proposición 2.6, $C_{p}(\xi)$ no es débilmente Whyburn.

2) Si $\kappa$ es $\omega$-inaccesible entonces $C_{p}(\xi)$ es débilmente Whyburn.

Tomando en cuenta el Lema 3.18, debemos demostrar que si $A \subseteq C_{p}(\xi)$ es un subconjunto no cerrado tal que $\overline{0} \in \operatorname{cl}_{C_{p}(\xi)}(A) \backslash A \operatorname{y~cl}_{C_{p}(\xi)}(B) \subseteq A$ para cada $B \in[A]^{<\kappa}$, entonces existe un conjunto casi cerrado $M \subseteq A$ con un único punto de acumulación en $C_{p}(\xi) \backslash A$.

Fijamos un conjunto cofinal $C$ en $\xi$ de cardinalidad $\kappa$. Sea $C=\left\{\eta_{\alpha}: \alpha \in\right.$ $\kappa\}$ tal que si $\alpha<\beta$ entonces $\eta_{\alpha}<\eta_{\beta}$. Para cada $\alpha \in \kappa$, sea $R_{\alpha}=\left\{\left.f\right|_{\eta_{\alpha}+1}\right.$ : $f \in A\}$.

Para $g \in R_{\alpha}, e(g)$ denota un elemento de $A$ que extiende a $g$, es decir, $\left.e(g)\right|_{\eta_{\alpha}+1}=g$.

Obsérvese que dado que $c f\left(\eta_{\alpha}+1\right)=1 \leq \omega$ para cada $\alpha \in \kappa, C_{p}\left(\eta_{\alpha}+1\right)$ es Fréchet, por el Teorema 3.17. Entonces existe un conjunto numerable $D_{\alpha} \subseteq$ $R_{\alpha}$ que converge puntualmente como sucesión a $\left.\overline{0}\right|_{\eta_{\alpha}+1}$.

Sea $E_{\alpha}=e\left(D_{\alpha}\right)$. Denotemos $E_{\alpha}=\left\{g_{i}^{\alpha}: i \in \omega\right\}$. Como la sucesión de funciones $\left.g_{i}^{\alpha}\right|_{\eta_{\alpha}+1}$ converge puntualmente a la función $\left.\overline{0}\right|_{\eta_{\alpha}+1}$, se tiene en particular que $\overline{0} \in \mathrm{cl}_{C_{p}(\xi)}\left(\left\{g_{i}^{\alpha}: i \in \omega \mathrm{y} \alpha \in \kappa\right\}\right)$. Sin perder generalidad, podemos suponer lo siguiente: 
(a) Para cada $i \in \omega$ y para cada $\alpha \in \kappa$ se tiene que $\left|g_{i}^{\alpha}(0)\right|<\frac{1}{i+1}$.

Observe que podemos suponer que existe $\alpha_{0} \in \kappa$ tal que para toda $\alpha \geq \alpha_{0}$, $D_{\alpha}$ es infinito. De otro modo, si $D_{\alpha}$ es finito entonces $\left.\overline{0}\right|_{\eta_{\alpha}+1} \in D_{\alpha}$ para cada $\alpha \in L$ donde $L$ es una familia cofinal en $\kappa$. Para cada $\alpha \in L$, sea $h_{\alpha} \in A$ tal que $\left.h_{\alpha}\right|_{\eta_{\alpha}+1}$ entonces $\left\{h_{\alpha}\right\}_{\alpha \in \kappa} \rightarrow \overline{0}$. Por tanto, $\overline{0}$ es el único punto de acumulación de $A$ en $C_{p}(\xi) \backslash A$, lo que concluiría la prueba. Así que podemos suponer que $D_{\alpha}$ es infinito para cada $\alpha \in \kappa$.

Para cada $i \in \omega$ y para toda $\alpha \in \kappa$, sea $A_{\alpha}^{i}=J\left(g_{i}^{\alpha}\right)$. Por el Lema 3.19, existe $D \in[\kappa]^{\kappa}$ tal que $\left\{A_{\alpha}^{i}: \alpha \in D\right\}$ forma un $\Delta$-sistema para cada $i \in \omega \mathrm{y}$ las raíces correspondientes las denotaremos como $R_{i}$.

Recordando que $k>2^{\omega}$ porque $\kappa$ es $\omega$-inaccesible, que todas las $R_{i}$ son numerables y que hay precisamente $2^{\omega}$ funciones de $R_{i}$ en $\mathbb{R}$, podemos suponer que:

(b) Para cada $\alpha, \alpha^{\prime} \in D$ e $i \in \omega$ se tiene.

$$
\left.\delta_{g_{i}^{\alpha}}\right|_{R_{i}}=\left.\delta_{g_{i}^{\alpha^{\prime}}}\right|_{R_{i}} \text { y }\left.g_{i}^{\alpha}\right|_{R_{i} \cup\{0\}}=\left.g_{i}^{\alpha^{\prime}}\right|_{R_{i} \cup\{0\}}
$$

pues de las $\kappa$ funciones distintas sólo hay $\mathfrak{c}$ funciones con valores reales definidas en $R_{i}$ o bien en $R_{i} \cup\{0\}$.

Para simplificar la notación, escribiremos $\delta_{i}: R_{i} \rightarrow \mathbb{R}$ en el lugar de $\delta_{\left.g_{i}^{\alpha}\right|_{R_{i}}}: R_{i} \rightarrow \mathbb{R}$. Además, para $i \in \omega$, sea $M_{i}=\left\{g_{i}^{\alpha}: \alpha \in D\right\}$ y $M=\bigcup\left\{M_{i}\right.$ : $i \in \omega\}$.

Afirmación 6. Si $\varphi \in \operatorname{cl}_{C_{p}(\xi)}\left(M_{i}\right) \backslash A$ entonces $J(\varphi)=R_{i}$. Además, $\delta_{\varphi}$ es precisamente $\delta_{i}$.

Supongamos que $\varphi \in c l_{C_{p}(\xi)}\left(M_{i}\right) \backslash A$. Notemos que $\gamma \in J(\varphi)$ si y sólo si $\gamma \in J(\varphi, \varepsilon)$ para algún $\varepsilon>0$ si y sólo si existe $\varepsilon>0$ tal que $|\varphi(\gamma)-\varphi(\gamma+1)|$ $>\varepsilon$ si y sólo si $\varphi(\gamma) \neq \varphi(\gamma+1)$.

Como $\varphi \in \mathrm{cl}_{C_{p}(\xi)}\left(M_{i}\right)$ y la convergencia en $M_{i}$ es puntual, se tiene que si $\varphi(\gamma) \neq \varphi(\gamma+1)$, entonces existen $\alpha_{1}, \alpha_{2} \in D$ tales que $\gamma \in A_{\alpha_{j}}^{i}=J\left(g_{\alpha_{j}}^{i}\right)$ para $j=1,2$. Por tanto, $\gamma \in R_{i}=A_{\alpha_{1}}^{i} \cap A_{\alpha_{2}}^{i}$.

Si $\gamma \in R_{i}$ entonces $\gamma \in A_{\alpha}^{i}$ para cada $\alpha \in \kappa$, de donde $g_{i}^{\alpha}(\gamma) \neq g_{i}^{\alpha}(\gamma+1)$ para cada $\alpha \in \kappa$. Así, $\gamma \in J(\varphi)$ puesto que $\delta_{i}(\gamma) \neq 0$. Además, como 
$\varphi \in c l_{C_{p}(\xi)}\left(M_{i}\right)$, se tiene que $\delta_{\varphi}=\delta_{i}$, por la propiedad (b).

Como $\varphi$ está únicamente determinado por $\varphi(0), J(\varphi)$ y $\delta_{\varphi}$, la Afirmación 6 implica que $c l_{C_{p}(\xi)}\left(M_{i}\right) \subseteq A$ o bien que $\left|c l_{C_{p}(\xi)}\left(M_{i}\right) \backslash A\right|=1$. Si $\left|c l_{C_{p}(\xi)}\left(M_{i}\right) \backslash A\right|=1$ entonces el conjunto $c_{C_{p}(\xi)}\left(M_{i}\right) \cap A$ es el conjunto casi cerrado requerido. De manera que supondremos que $c l_{C_{p}(\xi)}\left(M_{i}\right) \subseteq A$ para toda $i \in \omega$.

Afirmación 7. $c_{C_{p}(\xi)}(M) \cap A$ es casi cerrado y $\overline{0} \in c l_{C_{p}(\xi)}(M)$.

Sea $U=\left[\overline{0}, \alpha_{1}, \ldots, \alpha_{\kappa}, \varepsilon\right]$ cualquier vecindad básica de $\overline{0}$. Considerando que la sucesión $\left.g_{i}^{\alpha}\right|_{\eta_{\alpha}+1}$ converge puntualmente a $\left.\overline{0}\right|_{\eta_{\alpha}+1}$, se tiene que $\left\{g_{i}^{\alpha}: \alpha \in\right.$ $D\}$ converge uniformemente a $\overline{0}$ en cualquier subconjunto finito de $\eta_{\alpha}+1$; en particular, converge sobre el conjunto $\left\{\alpha_{1}, \ldots, \alpha_{\kappa}\right\}$. Esto es, $U \cap M_{i} \neq \emptyset$ para alguna $i \in \omega$. Luego $\overline{0} \in c_{C_{p}(\xi)}(M)$. Para probar que $c l_{C_{p}(\xi)}(M) \cap A$ es casi cerrado, supongamos que existe $\psi \in \operatorname{cl}_{C_{p}(\xi)}(M) \backslash A$ donde $\psi \neq \overline{0}$.

Caso 1. Supongamos que $\psi$ es constante. Por la propiedad (a), existe $n \in$ $\omega$ tal que $\frac{1}{n}<|\psi(0)|$. Tomamos una vecindad $U$ de $\psi$ tal que $U \cap$ $\left(\bigcup_{i=0}^{n} M_{i}\right)=\emptyset$. Sea $\varepsilon=\frac{1}{4}\left(|\psi(0)|-\frac{1}{n}\right)$ y tomemos $O=\left\{h \in C_{p}(\xi)\right.$ : $|h(0)-\psi(0)|<\varepsilon\}$. Finalmente, si $V=U \cap O$ entonces $V$ es una vecindad de $\psi$. Además, puesto que para cada $h \in M_{i}$ se tiene que $|h(0)|<\frac{1}{1+i}$, tomamos $i>2 n-1$. De manera que para cada $h \in M$ se tiene que $|h(0)|<\frac{1}{2 n}$, lo que implica que $V \cap M=\emptyset$.

Caso 2. Supongamos que $\psi$ no es constante. Entonces $J(\psi) \neq \emptyset$. Tomemos $\mu \in J(\psi)$ y $\varepsilon=\frac{1}{4}\left|\delta_{\psi}(\mu)\right|$. Sean

$$
S=\left\{i \in \omega: \mu \notin R_{i}\right\} \quad \text { y } F=\left\{g \in M_{i}: i \in S \text { y } \mu \in J(g)\right\} .
$$

Nótese que $i \in S$ si y sólo si $\mu \notin R_{i}$, lo que implica que $\mid\{\alpha: \mu \in$ $\left.A_{\alpha}^{i}\right\} \mid \leq 1$ si y sólo si $\left|\left\{\alpha: g_{\alpha}^{i}(\mu) \neq g_{\alpha}^{i}(\mu+1)\right\}\right| \leq 1$ si y sólo si $\left|\left\{\alpha: \mu \in J\left(g_{\alpha}^{i}\right)\right\}\right| \leq 1$ si y sólo si para cada $i \in \mathbb{N}$ hay a lo más una función $g \in M_{i}$ tal que $i \in S$ y $\mu \in J(g)$.

De modo que $F$ es a lo más numerable y, por hipótesis, $c l_{C_{p}(\xi)}(F) \subset A$. Como $\psi \notin A$ se sigue $\psi \notin c l_{C_{p}(\xi)}(F)$, entonces existe un abierto $U$ que contiene a $\psi$ tal que $U \cap F=\emptyset$. 
Sea $W=\left\{f \in C_{p}(\xi):|f(\mu+\zeta)-\psi(\mu+\zeta)|<\varepsilon, \zeta=0,1\right\}$. Entonces, $\left(\bigcup\left\{M_{i}: i \in S\right\} \backslash F\right) \cap W=\emptyset$. En efecto, supongamos que $g \in \bigcup\left\{M_{i}: i \in S\right\} \backslash F$. Por definición de $F$ se tiene que $\mu \notin J(g)$ y, en consecuencia, $g(\mu)=g(\mu+1)$. Por lo que, $g \notin W$. En particular, $\psi \notin c l_{C_{p}(\xi)}\left(\bigcup\left\{M_{i}: i \in S\right\}\right)$.

Sea $T=\omega \backslash S=\left\{i \in \omega: \mu \in R_{i}\right\}$. Entonces, $\psi \in \operatorname{cl}_{C_{p}(\xi)}\left(\bigcup\left\{M_{i}: i \in T\right\}\right)$ lo que implica que $T$ es infinito. Afirmamos que $\lim _{i \rightarrow \infty}\left\{\delta_{i}(\mu): i \in\right.$ $T\}=0$. De lo contrario, existen una subsucesión $T^{\prime} \subset T$ y $k<\omega$ tales que $\left|\delta_{i}(\mu)\right|>\frac{1}{k}$ para toda $i \in T^{\prime}$. Tomamos cualquier $\alpha$ tal que $\eta_{\alpha}>\mu+1$. Por construcción, sabemos que $D_{\alpha}$ converge a $\left.\overline{0}\right|_{\eta_{\alpha}+1}$. Debido a la convergencia puntual, existe $i_{\alpha} \in \omega$ tal que para cada $i \in i_{\alpha}$ con $i \in T^{\prime}$ se tiene que el máx $\left(\left|g_{i}^{\alpha}(\mu)\right|,\left|g_{i}^{\alpha}(\mu+1)\right|\right)<\frac{1}{3 k}$, lo cual es una contradicción.

Tomamos $m_{0} \in \omega$ tal que cada $m>m_{0} \operatorname{con} m \in T$ sucede que $\left|\delta_{m}(\mu)\right|$ $<\varepsilon$. Sea $V$ una vecindad de $\psi$ tal que $V \cap\left(\bigcup_{i=0}^{m_{0}} M_{i}\right)=\emptyset$. Recordando que la vecindad $W=\left\{f \in C_{p}(\xi):|f(\mu+\zeta)-\psi(\mu+\zeta)|<\varepsilon, \zeta=0,1\right\}$ se sigue que $W \cap\left(\bigcup\left\{M_{i}: i \in T\right.\right.$ e $\left.\left.i>m_{0}\right\}\right)=\emptyset$, ya que si $g_{\alpha}^{i} \in W$ entonces $\left|g_{\alpha}^{i}(\mu)-g_{\alpha}^{i}(\mu+1)\right|>2 \varepsilon$. Sea $Q=U \cap V \cap W$. Entonces $Q \cap M=\emptyset$, lo que contradice que $\psi \in \operatorname{cl}_{C_{p}(\xi)}(M)$.

3.21 Corolario $([9]) . C_{p}(\xi)$ es Whyburn si y sólo si cf $(\xi) \leq \omega$.

Demostración. Supongamos que $c f(\xi) \leq \omega$. Por el Teorema 3.17, $C_{p}(\xi)$ es de Fréchet y por la Proposición 2.4.1, $C_{p}(\xi)$ es Whyburn.

Supongamos que $C_{p}(\xi)$ es Whyburn y sea $c f(\xi)=\kappa$. Sin pérdida de generalidad podemos suponer que $\kappa$ es infinito. Puesto que $C_{p}(\kappa)$ es un subconjunto cerrado de $C_{p}(\xi)$, se tiene que $C_{p}(\kappa)$ es de Whyburn. Dado que $C_{p}(\xi)$ es débilmente de Whyburn, el Teorema 3.20, implica que $\kappa=\omega$ o que $\kappa$ es $\omega$-inaccesible. Además, por el Teorema 3.16, $C_{p}(\kappa)$ es pseudoradial y, por el Teorema 2.30, es radial. Ahora, si aplicamos el Teorema 3.17, vemos que $\kappa=\omega$. 


\section{Preguntas Abiertas}

1. [7] ¿Existe un espacio compacto y pseudoradial que no sea débilmente Whyburn?

2. [7] ¿El producto de dos espacios (numerables) compactos y débilmente Whyburn sigue siendo débilmente Whyburn?

3. [7] Suponiendo que $C_{p}(\xi)$ es Whyburn. ¿El espacio $C_{p}(\xi)$ tiene estrechez numerable?

4. [21] ¿Es cierto que cualquier subespacio de un espacio secuencial es débilmente Whyburn?

5. [21] Suponiendo que $C_{p} C_{p}(X)$ es Whyburn. ¿Es cierto que $X$ es finito?

6. [21] ¿El espacio $C_{p}\left(\mathbb{R}^{\omega}\right)$ es débilmente Whyburn?

7. [21] Suponiendo que $X$ es un espacio segundo numerable y $C_{p}(X)$ es Whyburn, ¿Todas las potencias finitas de $X$ son espacios de Hurewicz?

8. [21] Suponiendo que $C_{p}(X)=A \cup B$; donde $A$ y $B$ son espacios de Whyburn. ¿Es cierto que $C_{p}(X)$ es un espacio Whyburn?

9. [21] Suponiendo que $C_{p}(X)$ es un espacio Whyburn. ¿Es cierto que $\left(C_{p}(X)\right)^{\omega}$ es un espacio Whyburn?

10. [18] ¿Es cierto que bajo $M A+\neg C H$, cualquier espacio de Hausdorff, numerablemente compacto y de carácter $\leq \omega_{1}$ es débilmente Whyburn? ¿Qué sucede si omitimos numerablemente compacto? 
11. [18] ¿Es cierto que bajo $M A+\neg C H$, cualquier espacio (Tychonoff) con peso $\leq \omega_{1}$ es débilmente Whyburn?

12. [18] ¿Es cierto que cualquier $P$-espacio (regular) de cardinalidad $\leq \omega_{1}$ es un espacio (débilmente) Whyburn?

13. [18] ¿Para qué espacios compactos $X$, la $\omega$-modificación de $X$ es un espacio débilmente Whyburn?

¿La $\omega$-modificación de $\beta \omega \backslash \omega$ es un espacio débilmente Whyburn? 


\section{Bibliografía}

[1] O.T. Alas, R.G. Wilson, Weaker Connected Hausdorff Topologies on Spaces with a $\sigma$-Locally Finite Base, Houston Journal of Math. 31 (2005), no. 2, 427-439.

[2] A.V. Arhangel'skii, Bicompact sets and the topology of spaces, Soviet Mathematics, Doklady 4 (1963), 561-564.

[3] A.V. Arhangel'skii, A characterization of very $k$-spaces, Czechoslovak Math. J. 18 (1968), 392-395.

[4] A.V. Arhangel'skii, Topological Function Spaces, Kluwer Academic Publishers, Mathematics and Its Applications 78, Dordrecht 1992.

[5] Z. Balogh, On compact Hausdorff spaces of countable tightness, Proc. Amer. Math. Soc. 105 (1989), 755-764.

[6] L.A. Steen and J.A. Seebach, Jr., Counterexamples in topology, second edition, Dover Publications, Inc. New York, 1995.

[7] A. Bella, I.V. Yaschenko, On AP and WAP spaces, Comment. Math. Univ. Carolinae, 40 (1999), no. 3, 531-536.

[8] A. Bella, On spaces with the property of weak approximation by points, Comment. Math. Univ. Carolinae, 35 (1994), no. 2, 357-360.

[9] A. Bella, Weakly Whyburn spaces of continuous functions on ordinals, Topology Appl. 133 (2003), no. 1, 97-104.

[10] E.K. van Douwen, The integers and topology, Handbook of Set-Theoretic Topology, ed. por K. Kunen y J. E. Vaughan, North Holland P. C., Amsterdam, 111-167. 
[11] R. Engelking, General Topology, Heldermann Verlag, Berlin, 1989.

[12] V.V. Fedorchuk, Fully closed mappings and the consistency of some theorems of general topology with the axioms of set theory, Mathematics in the USSR, Sbornik, 28 (1976), 1-26.

[13] J. Gerlits, Z. Nagy, Z. Szentmiklossy, Some convergence properties in function spaces, in: Z. Frolík (Ed.), General Topology and its Relations to Modern Analysis and Algebra VI, in: Res. Exp. Math., Vol. 16, Heldermann, Berlin (1988), 211-222.

[14] R. Hodel, Cardinal Functions I, Handbook of Set-Theoretic Topology, ed. por K. Kunen y J.E. Vaughan, North Holland P.C., Amsterdam, $1-61$.

[15] I. Juhász, Cardinal functions in topology - Ten years later, Math. Centre Tracts 123, Amsterdam 1980.

[16] F. Obersnel, Open maps do not preserve Whyburn property, Comment. Math. Univ. Carolinae, 44 (2003), no. 3, 525-530.

[17] F. Obersnel, Some notes on weakly Whyburn spaces, Topology Appl. 128 (2003), no. 2-3, 257-262.

[18] J. Pelant, M.G. Tkachenko, V.V. Tkachuk, R.G. Wilson, Pseudocompact Whyburn spaces need not be Fréchet, Proc. Amer. Math. Soc. 131 (2003), no. 10, 3257-3265.

[19] A. Pultr y A. Tozzi, Equationally closed subframes and representations of quotient spaces, Cahiers de Topologie et Geometrie Differentielle Categoriques 34 (1993), 167-183.

[20] P. Simon, On accumulation points, Cahiers Topologie Géom. Différentielle Categoriques, 35 (1994), 321-327.

[21] V.V. Tkachuk, I.V. Yashenko, Almost closed sets and topologies they determine, Comment. Math. Univ. Carolinae, 42 (2) (2001), 395-405.

[22] G.T. Whyburn, Accessibility spaces, Proceedings Amer. Math. Soc. 24 (1970), 181-185. 


\title{
UNIVERSIDAD AUTÓNOMA METROPOLITANA
}

\section{ESPACIOS DE WHYBURN}

\author{
Presenta \\ MAIRA MADRIZ MENDOZA \\ para la obtención del título de \\ MAESTRA EN CIENCIAS \\ (MATEMÁTICAS) \\ Director de Tesis: \\ Dr. Richard Wilson Roberts

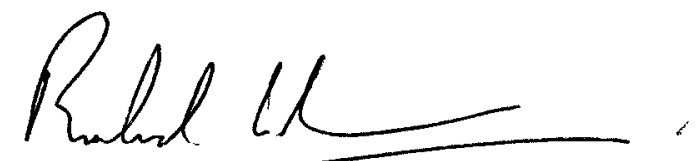 \\ Unidad Iztapalapa \\ Departamento de Matemáticas
}

México

1 de Octubre de 2007 